\title{
Dynamics and entropy in the Zhang model of Self-Organized Criticality
}

\author{
B. Kruglikov \& M. Rypdal \\ Institute of Mathematics and Statistics \\ University of Troms $\varnothing$, N-9037 Troms $\varnothing$, Norway \\ Boris.Kruglikov@matnat.uit.no; Martin.Rypdal@matnat.uit.no
}

\begin{abstract}
We give a detailed study of dynamical properties of the Zhang model, including evaluation of topological entropy and estimates for the Lyapunov exponents and the dimension of the attractor. In the thermodynamic limit the entropy goes to zero and the Lyapunov spectrum collapses.
\end{abstract}

\section{Introduction}

In 1987 the concept of Self-Organized Criticality (SOC) was introduced by Bak, Tang and Wiesenfeld BTW]. The attempt was to give an explanation of the omnipresence of fractal structures and power-law statistics in nature, and the claim was that certain physical systems can self-organize into stationary states, reminiscent of equilibrium system at the critical point, in the sense that one has scale invariance and long range correlations in space and time.

SOC is proposed as an explanation for variety of phenomena in nature, such as earthquakes, forest fires, stock markets and biological evolution [J]. However, most work has been devoted to the study of idealized "sandpile-like" computer models, such as the sandpile model [BTW], the abelian sandpile [DR] and the Zhang model [Z] that, one believes, exhibit SOC in the thermodynamic limit. Despite this effort, a satisfactory understanding of the model is not yet achieved. Through numerical investigation it was observed that in the thermodynamic limit, observables have power-law distributions. More precisely, the probability distribution of an observable $s$ has the form $P(s) \sim 1 / s^{\tau_{s}}$ in the thermodynamic limit. There is no widely agreed upon method for computing the SOC-exponents $\tau_{s}$ numerically and, due to the incomplete understanding of the dynamics of the models and lack of a formal treatment of the thermodynamic limit, it is difficult to properly explain the observed behavior. Hence it is not clear what the SOCexponents really tell us about the dynamics of the SOC models.

\footnotetext{
${ }^{1}$ Keywords: sand-pile models, avalanche dynamics, skew-product systems, Lyapunov exponents, entropy, Hausdorff dimension, thermodynamic limit.
} 


\section{Boris Kruglikov, Martin Rypdal}

\subsection{Discussion of the Zhang model}

In a series of papers by Cessac, Blanchard and Krüger BCK] it was proposed that deeper understanding of SOC models can be achieved by studying the models in the framework of dynamical system theory. They showed how a particular model, the Zhang model, could be formulated as a dynamical system of skew-product type with singularities, where the randomness of the external driving is described by a Bernoulli shift, and the threshold relaxation dynamics is given by piecewise affine maps.

In this paper we present a detailed study of the dynamical system defined in $\mathrm{BCK}$. We prove several basic properties, some of which are already stated in $[\mathrm{BCK}$, before discussing fundamental dynamical properties. Depending on the parameters of the model, we can observe fundamentally different types of behavior.

For low values of the threshold energy (critical energy), the dynamics can be relatively simple, since the singularities only effect the dynamics in a finite number of time-steps. In such situations we say that singularities are removable, and we show that the system permits symbolic coding. We give examples of how symbolic coding provides a complete description of the dynamics as a topological Markov chain. Hence the dynamics is chaotic, but the essential dynamical invariants are all inherited from the Bernoulli shift factor. Moreover we can identify the physical invariant measure, and hence understanding of the statistical properties is reduced to the theory of Markov chains.

As we increase the critical energy the role of the singularities becomes essential. Techniques based on codings are no longer applicable and a very interesting dynamics emerges. The dimensional characteristics of the attractor are also sensitive to the parameters of the system, as we show by generalizing the Moran formula for the iterated function system (IFS). In addition, we observe the situation, when the dimension of the IFS-attractor increases to the maximum, while the support of the SRB-measure remains fractal.

To measure the complexity of the dynamics we study entropy and Lyapunov exponents. We show that the system is hyperbolic, with one positive exponent originating in the Bernoulli shift. However, due to the presence of singularities the Ruelle inequality and the Pesin formula are not directly applicable. We show that the metric entropy of any SRB-measure equals the topological entropy almost surely, and we evaluate the latter generalizing the technique developed by Buzzi [B1, B2. The result is that the Pesin formula and the variational principle hold a posteriori.

To give a satisfactory physical interpretation of the dynamics we rescale time to prevent infinitely slow driving of the system. We prove that for this physical system, the Lyapunov spectrum collapses completely and that the entropy goes to zero in the thermodynamic limit. This implies that the expanding (chaotic) properties are lost, so that we may expect power-laws statistics and long range correlation effects.

The statistical properties we obtain hold for any SRB-measure, because the most input comes from the Bernoulli shifts. The existence of SRB-measures is 
Dynamics and entropy of SOC

in fact still an open problem. From the general theory of dynamical systems with singularities [KS $, \mathrm{P} 1, \mathrm{ST}]$, we can give conditions that are sufficient for the existence of SRB-measures, but it is not known if these conditions hold for the majority of parameters. We expect this to be true (it was also conjectured in BCK ) and derive some statistical corollaries.

Apart from the physical importance of the Zhang model, it is interesting from a mathematical point of view. It can be described as a piecewise affine hyperbolic map of the form

$$
F: \Sigma_{N}^{+} \times M \rightarrow \Sigma_{N}^{+} \times M, \quad\left(\left(t_{0} t_{1} t_{2} \ldots\right), x\right) \mapsto\left(\left(t_{1} t_{2} t_{3} \ldots\right), f_{t_{0}}(x)\right),
$$

where $\Sigma_{N}^{+}$is the set of right infinite sequences from a finite alphabet and $\left\{f_{i}\right\}$ a collection of piece-wise affine non-expanding maps of $M$ to itself. Previously piecewise affine expanding maps and piecewise isometries have been studied, but the contracting property of the relaxation dynamics gives rise to some difficulties. Therefore several methods are developed in this paper, which hold far beyond the framework of the Zhang model.

\subsection{Structure of the paper}

In Section 1 we describe the model and derive bounds on the size and duration of avalanches. This enables us to use the Poincaré return to reformulate the systems in a skew-product form. Then we study the contraction property to conclude hyperbolicity of the model (Theorem 5) and describe, when degenerations occur (Theorem 8 the original Zhang setting $\epsilon=0$ is not the only possibility). In Section 2 we introduce the concept of removability of singularities, which appears in the coding approach for the study of the model.

Section 3 is devoted to the study of measure entropy and Lyapunov spectrum. We prove in Theorem 13 that the entropy of an SRB-measure is always maximal. Section 4 concerns the topological entropy. We evaluate it for the most parameter values (Theorems [18 and 19). We also discuss nearly-Zhang models and show that the dynamical quantities do not change. This is natural from the physical perspective, because SOC should not be obtained through a fine tuning of parameters.

Section $[5$ briefly describes the dimension issues of the model (Theorem 20] gives the asymptotic values), which enters into all inter-relations involving entropy and characteristic exponents. We demonstrate how the fractality occurs in IFS-context, noting the difference due to singularities and overlaps. In Section [6] we illustrate the most important effects in the model by examples.

In Section 7 we discuss the thermodynamic limit and attempt to explain appearance of the power-law statistics via a reparametrization. Conclusion contains the physical implications of the current investigation.

In Appendices $\mathrm{A}$ and $\mathrm{B}$ we provide bounds for the entropy and the dimension, which are new in the presence of singularities, overlaps and degenerations (this was designed for an application to the Zhang model). The results are of interest in its own and can be read independently. 


\section{Basic properties of the Zhang model}

In the Zhang model each site on the lattice is associated with a non-negative real number, which we call the energy of the site. The collection of energies is called an energy configuration, and can be represented as a point in $N$-dimensional space, where $N$ is the number of sites in the lattice. If a configuration is unstable, the overcritical sites will lose some of their energy to their nearest neighbors, resulting in a new energy configuration. This transformation on $\mathbb{R}^{N}$ is denoted by $f$. If a configuration is stable, a site is chosen at random and an energy quantum $\delta=1$ is added to this site. In [BCK] it was shown how the relaxation and random excitation can be formulated as a map of skew-product type on an extended phase-space. This extended phase-space has the configuration space as one factor, and the set of all possible sequences of excitations as the other factor. In BCK] it was also shown how one can reformulate the dynamical system by considering the return maps to the set of stable configurations. This gives a simplification, in the sense that each avalanche is associated with an affine transformation. The set of stable configurations is partitioned into domains, where each domain corresponds to an avalanche.

\subsection{Relaxation}

Take $d, L \in \mathbb{N}$ and let $\Lambda \subset \mathbb{Z}^{d}$ be the cube $[1, L]^{d}$ of cardinality $N:=L^{d}=|\Lambda|$. Let $\phi: \Lambda \rightarrow \Lambda^{\prime}:=\{1, \ldots, N\}$ be a bijection. We define a metric $d_{\Lambda}$ on $\Lambda$ by

$$
d_{\Lambda}(\mathbf{k}, \mathbf{l})=\sum_{1 \leq n \leq d}\left|k_{n}-l_{n}\right|
$$

and let $d_{\Lambda^{\prime}}:=\phi_{*} d_{\Lambda}$. In the following we omit primes when it is clear from the context that we are considering the metric space $\left(\Lambda^{\prime}, d_{\Lambda^{\prime}}\right)$. Elements of $\Lambda$ will be called sites. We say that sites $i$ and $j$ are nearest neighbors if $d_{\Lambda}(i, j)=1$. The boundary $\partial \Lambda$ is defined as those sites $i \in \Lambda$ that have less than $2 d$ nearest neighbors.

Fix parameters $E_{c}>0$ and $\epsilon \in[0,1)$ and define $f: \mathbb{R}_{\geq 0}^{N} \rightarrow \mathbb{R}_{\geq 0}^{N}$ by

$$
f(x)_{i}=x_{i}-\theta\left(x_{i}-E_{c}\right)(1-\epsilon) x_{i}+\frac{1-\epsilon}{2 d} \sum_{d_{\Lambda}(i, j)=1} \theta\left(x_{j}-E_{c}\right) x_{j},
$$

where

$$
\theta(a)=\left\{\begin{array}{l}
1 \text { if } a>0 \\
0 \text { if } a \leq 0
\end{array} .\right.
$$

Let $\|x\|_{1}=\sum_{i=1}^{N}\left|x_{i}\right|$ be the 1 -norm on $\mathbb{R}^{N}$.

Proposition 1. For all $x \in \mathbb{R}_{\geq 0}^{N}$ we have

$$
\frac{1+\epsilon}{2}\|x\|_{1} \leq\|f(x)\|_{1} \leq\|x\|_{1},
$$


and $\|f(x)\|_{1}=\|x\|_{1}$ if and only if $x_{i} \leq E_{c}$ for all $i \in \partial \Lambda$. If there is $i \in \partial \Lambda$ such that $x_{i}>E_{c}$ then

$$
\|f(x)\|_{1} \leq\|x\|_{1}-\frac{1-\epsilon}{2 d} E_{c} .
$$

Proof. Let $\left\{x_{i_{k}}\right\}_{k=1}^{m}$ be the entries of the vector $x$ that are greater than $E_{c}$. Let $n_{i_{k}}$ be the number of nearest neighbors of $x_{i_{k}}$. Then

$$
\begin{aligned}
\|f(x)\|_{1} & =\sum_{i \in \Lambda} f(x)_{i}=\sum_{i \in \Lambda} x_{i}-(1-\epsilon) \sum_{k=1}^{m} x_{i_{k}}+\frac{1-\epsilon}{2 d} \sum_{k=1}^{m} n_{i_{k}} x_{i_{k}} \\
& =\sum_{i \in \Lambda} x_{i}-(1-\epsilon) \sum_{k=1}^{m}\left(1-\frac{n_{i_{k}}}{2 d}\right) x_{i_{k}} .
\end{aligned}
$$

The statement follows from the fact that we always have $d \leq n_{i_{k}} \leq 2 d$, and $n_{i_{k}}=2 d$ if and only if $x_{i_{k}} \notin \partial \Lambda$.

We say that a site $i \in \Lambda$ of the configuration $x$ is relaxed if $x_{i} \leq E_{c}$, and excited if $x_{i}>E_{c}$. A configuration $x$ is called stable if all sites are relaxed. The set of stable configurations is $M:=\left[0, E_{c}\right]^{N}$. For each configuration $x$ we define $m(x)=\min \left\{n \geq 0 \mid f^{n}(x) \in M\right\}$.

Proposition 2. For all $x \in \mathbb{R}_{\geq 0}^{N}$ we have:

$$
m(x) \leq \frac{2 d N}{1-\epsilon} \frac{\|x\|_{1}}{E_{c}}\left(\frac{2 d}{1-\epsilon}+1\right)^{\operatorname{diam}(\Lambda) / 2} .
$$

We need the following lemma ([.] denotes integer part):

Lemma 3. For $x \in \mathbb{R}_{\geq 0}^{N}$ and $n \in \mathbb{N}$ let $\alpha_{i}(n, x)$ be the cardinality of the set $\left\{l \leq n \mid\left(f^{l} x\right)_{i}>E_{c}\right\}$. Let $\gamma=[2 d /(1-\epsilon)]+1$. If $d_{\Lambda}(i, j)=1$, then $\alpha_{j}(n, x) \geq$ $\left[\alpha_{i}(n, x) / \gamma\right]$.

Proof. There is a finite increasing sequence $\left\{m_{k}\right\}$, such that $\left(f^{m_{k}}(x)\right)_{i}>E_{c}$. We claim that on each interval $\left(m_{k}, m_{k+\gamma}\right]$ there is a number $m$ such that $\left(f^{m}(x)\right)_{j}>E_{c}$. In fact, in the opposite case

$$
\left(f^{1+m_{k+\gamma-1}}(x)\right)_{j} \geq \gamma \frac{1-\epsilon}{2 d} E_{c}>E_{c} .
$$

Since $\left[0, \alpha_{i}(n, x)\right]$ contains $\beta=\left[\alpha_{i}(n, x) / \gamma\right]$ disjoined such intervals, we get $\alpha_{j}(n, x) \geq \beta$. Thus $\alpha_{j}(n, x) \geq\left[\alpha_{i}(n, x) / \gamma\right]$.

Proof of Proposition 2, By applying inductively Lemma 3 we get:

$$
\alpha_{j}(n, x) \geq\left[\frac{\alpha_{i}(n, x)}{\gamma^{d_{\Lambda}(i, j)}}\right]
$$


In fact, if $j=j_{0}, j_{1}, \ldots j_{k}=i$ is a path with $d_{\Lambda}\left(j_{s}, j_{s+1}\right)=1$ and

$$
\left[\frac{\alpha_{i}(n, x)}{\gamma^{k}}\right]=t
$$

then $\alpha_{j_{k}}(n, x) \geq t \gamma^{k}, \alpha_{j_{k-1}}(n, x) \geq t \gamma^{k-1}, \ldots, \alpha_{j_{0}}(n, x) \geq t$. By Proposition 1

$$
\alpha_{j}(n, x) \leq \frac{2 d}{1-\epsilon} \frac{\|x\|_{1}}{E_{c}}
$$

for $j \in \partial \Lambda$, so

$$
\alpha_{i}(n, x) \leq \alpha(x):=\frac{2 d}{1-\epsilon} \frac{\|x\|_{1}}{E_{c}} \gamma^{\operatorname{diam}(\Lambda) / 2} .
$$

for all $i \in \Lambda$ and all $n \in \mathbb{N}$. Suppose $f^{m}(x) \notin M$ for all $m \leq T$. If $T>N \alpha(x)$, then there must be a site $i \in \Lambda$ that is greater than $E_{c}$ for more than $\alpha(x)$ different times. This is impossible so $m(x) \leq N \alpha(x)$.

\subsection{Random excitations}

Define $\Sigma_{N}^{+}=\Lambda^{\mathbb{N}}$ to be the set of right-infinite $\Lambda$-sequences and let $\sigma_{N}^{+}: \Sigma_{N}^{+} \rightarrow$ $\Sigma_{N}^{+}$be the left shift. We define a map $\hat{f}: \Sigma_{N}^{+} \times \mathbb{R}_{\geq 0}^{N} \rightarrow \Sigma_{N}^{+} \times \mathbb{R}_{\geq 0}^{N}$ by

$$
\hat{f}(\mathbf{t}, x)=\left\{\begin{array}{ll}
\left(\sigma_{N}^{+} \mathbf{t}, x+e_{t_{0}}\right) & \text { if } x \in M \\
(\mathbf{t}, f(x)) & \text { if } x \notin M
\end{array},\right.
$$

where $e_{1}, \ldots, e_{N}$ is the standard basis in $\mathbb{R}^{N}$. We denote points in $\Sigma_{N}^{+} \times \mathbb{R}_{\geq 0}^{N}$ by $\hat{x}=(\mathbf{t}, x)$, and we define $\pi_{u}$ and $\pi_{s}$ to be the projections to $\Sigma_{N}^{+}$and $\mathbb{R}_{\geq 0}^{N^{N}}$ respectively.

Proposition 4. For all $\hat{x} \in \Sigma_{N}^{+} \times \mathbb{R}_{\geq 0}^{N}$ it holds:

$$
\min \left\{m \geq 0 \mid \forall i \in \Lambda \exists m^{\prime} \leq m:\left(\pi_{s} \circ \hat{f}^{m^{\prime}}(\hat{x})\right)_{i}>E_{c}\right\} \leq n\left(E_{c}, \epsilon, \Lambda\right),
$$

where

$$
n\left(E_{c}, \epsilon, \Lambda\right)=N\left(N E_{c}+2\right)\left(\left[\frac{2 d}{1-\epsilon}\right]+1\right)^{\operatorname{diam}(\Lambda)},
$$

Proof. In $N\left[E_{c}\right]+1$ time-steps, there must be an overcritical site. Since in the relaxation process there is always an overcritical site, then during arbitrary subsequent $N\left[E_{c}\right]+2$ time-steps an exited site can be found. Hence after $N \xi\left(N\left[E_{c}\right]+2\right)$ time-steps either all sites have been overcritical or there is a site that has been overcritical at least $\xi$ times. However it follows from the proof of Proposition 2 that if one site is overcritical

$$
\xi=\left(\left[\frac{2 d}{1-\epsilon}\right]+1\right)^{\operatorname{diam}(\Lambda)}
$$

times, then all sites have been overcritical at least once. 
For $x \in \mathbb{R}_{\geq 0}^{N}$ and $i \in \Lambda$ we define $\tau(i, x):=\min \left\{n \in \mathbb{N} \mid f^{n}\left(x+e_{i}\right) \in M\right\}$. Proposition 2 assures us that this number is finite and

$$
\max _{x \in M} \max _{i \in \Lambda} \tau(i, x) \leq \tau_{m}\left(E_{c}, \epsilon, \Lambda\right)=N^{2}\left(1+\frac{1}{N E_{c}}\right)\left(\frac{2 d}{1-\epsilon}+1\right)^{\operatorname{diam}(\Lambda) / 2+1} .
$$

Thus we observe that neither $n\left(E_{c}, \epsilon, \Lambda\right)$ nor $\tau_{m}\left(E_{c}, \epsilon, \Lambda\right)$ are uniformly bounded in $E_{c}$, but there is the following alternative:

There exists a constant $C_{0}$, not depending on the energy $E_{c}$, such that either $n\left(E_{c}, \epsilon, \Lambda\right) \leq C_{0}$ or $\tau_{m}\left(E_{c}, \epsilon, \Lambda\right) \leq C_{0}$.

In fact, we can set $C_{0}=3 N^{2}\left(\frac{2 d}{1-\epsilon}+1\right)^{\operatorname{diam}(\Lambda)+1}$. Thus we get that either relaxation happen sufficiently fast or all the sites keep being excited sufficiently often (uniformly in $E_{c}$ ).

But there does not exist such a bound uniform in $\epsilon$ or $N$.

\subsection{Return maps}

Let $\hat{x}=(\mathbf{t}, x) \in \Sigma_{N}^{+} \times M$. For $n=1, \ldots, \tau\left(t_{0}, x\right)$ define $C_{n}(\hat{x})=\left\{i \in \Lambda \mid\left(\pi^{s} \circ\right.\right.$ $\left.\left.\hat{f}^{n} \hat{x}\right)_{i}>E_{c}\right\}$, and $A(\hat{x})=\left(C_{1}(\hat{x}), \ldots, C_{\tau\left(t_{0}, x\right)}(\hat{x})\right)$. We call $A(\hat{x})$ the avalanche of the point $\hat{x}$. Let $\hat{M}:=\Sigma_{N}^{+} \times M$ and define an equivalence relation $\sim$ on $\hat{M}$ by

$$
\hat{x} \sim \hat{y} \Leftrightarrow A(\hat{x})=A(\hat{y}) .
$$

This gives a partition of $\hat{M}$. From the definition it is clear that $A(\hat{x})$ depends on $t_{0}$ and $x$ only. Hence partition elements are of the form $[i] \times M_{i j}$, where

$$
\forall i \in \Lambda: \bigcup_{j} M_{i j}=M
$$

and $[i]=\left\{\mathbf{t} \in \Sigma_{N}^{+} \mid t_{0}=i\right\}$ is the cylinder of the symbol $i$. We see that for each $i \in \Lambda$, the domains $M_{i 1}, M_{i 2}, \ldots$ are separated by segments of at most $N !^{\tau_{m}}=\exp \left(\tau_{m}\left(E_{c}, \epsilon, \Lambda\right) \log N !\right)$ hyperplanes. Hence we have a finite number of domains $M_{i 1}, \ldots, M_{i q_{i}}$ for each $i \in \Lambda$. By definition there is a unique avalanche for each partition element $[i] \times M_{i j}$. We denote this avalanche by $A_{i j}$. Its duration is $\tau_{i j}:=\tau(i, x)$, for $x \in M_{i j}$, and define its size to be $s_{i j}=\sum_{n=1}^{\tau_{i j}}\left|C_{n}\right|$.

We define the piecewise continuous map $F: \hat{M} \rightarrow \hat{M}$ by

$$
(\mathbf{t}, x) \mapsto\left(\sigma_{N}^{+} \mathbf{t}, F_{t_{0}} x\right)
$$

where $F_{i}(x):=f^{\tau(i, x)}\left(x+e_{i}\right)$. We define $F_{i j}:=\left.F_{i}\right|_{M_{i j}}$.

Remark 1. From a mathematical point of view the formulation $(\hat{M}, F)$ is a simplification compared to $\left(\Sigma_{N}^{+} \times \mathbb{R}_{\geq 0}^{N}, \hat{f}\right)$. However, the duration of avalanches are suppressed so that all avalanches have the same duration. This is not satisfactory from a physical point of view, and hence we call $(\hat{M}, F)$ the mathematical model and $\left(\Sigma_{N}^{+} \times \mathbb{R}_{\geq 0}^{N}, \hat{f}\right)$ the physical model. We will later make a rescaling of time in the physical model, so that the driving does not become infinitely slow in the thermodynamic limit. 
For each $x \in \mathbb{R}_{\geq 0}^{N}$ we define a matrix $Q(x)$ by

$$
Q_{k l}(x)= \begin{cases}\frac{1}{2 d} \theta\left(x_{l}-E_{c}\right) & \text { if } d_{\Lambda}(k, l)=1, \\ 0 & \text { otherwise }\end{cases}
$$

and a diagonal matrix $J(x)$ by $J_{k l}(x)=\left(1-(1-\epsilon) \theta\left(x_{l}-E_{c}\right)\right) \delta_{k l}$. Set

$$
S(x)=J(x)+(1-\epsilon) Q(x)
$$

and observe that $f(x)=S(x) x$. Let $x(1)=x+e_{i}$ and $x(n)=f(x(n-1))$ for $n \in\{2, \ldots, \tau(t, x)\}$. Then $F_{i}(x)=L_{i}\left(x+e_{i}\right)$, where

$$
L_{i}(x)=S(x(\tau(x, i))) \ldots S(x(1)) .
$$

If $x, y \in M_{i j}$, then $\tau(i, x)=\tau(i, y)$ and the same components of $x(n)$ and $y(n)$ are grater than $E_{c}$ for each $n=1, \ldots, \tau(t, x)$, so $L_{i}(x)=L_{i}(y)$. We define the linear map $L_{i j}:=L_{i}(x)$ for $x \in M_{i j}$. We get $\left.F_{i}\right|_{M_{i j}}(x)=L_{i j}\left(x+e_{i}\right)$.

Definition 1. A sequence $\left\{\left(i_{n}, j_{n}\right) \mid 1 \leq n \leq \theta\right\}$ is said to be admissible if

$$
\bigcap_{n=1}^{\theta}\left(F_{i_{n-1} j_{n-1}} \circ \cdots \circ F_{i_{1} j_{1}}\right)^{-1}\left(M_{i_{n} j_{n}}\right) \neq \emptyset,
$$

Theorem 5. For all $i, j\left\|L_{i j}\right\|_{1} \leq 1$. Moreover for every constant $c \in(0,1)$ there is a number $T \in \mathbb{N}$ such that for every $\theta>T$ and admissible sequence $\left\{\left(i_{n}, j_{n}\right) \mid 1 \leq n \leq \theta\right\}$ it holds:

$$
\left\|L_{i_{\theta} j_{\theta}} \ldots L_{i_{1} j_{1}}\right\|_{1}<c
$$

Proof. If $A$ is an $N \times N$ matrix we let $C_{k}(A)$ be its $k$-th column. Observe that for any matrices $A$ and $B$ we have the following formula:

$$
\left\|C_{k}(A B)\right\|_{1}=\sum_{l}\left\|C_{l}(A)\right\|_{1} B_{l k} .
$$

By the construction: $\left\|C_{k}(S(x))\right\|_{1} \leq 1$ for all $k \in \Lambda$. Hence $\left\|L_{i j}\right\|_{1} \leq 1$.

To prove the second statement we note that for $\epsilon>0$ the diagonal elements of the matrices $S(x)$ are non-zero and $\geq \epsilon$. Therefore

$$
(S(x(m)) S(x(m-1)))_{k l} \geq \epsilon \cdot \max \left\{S_{k l}(x(m)), S_{k l}(x(m-1))\right\} .
$$

Moreover, $S_{k l}(x(m))>0$ if $x(m)_{l}>0$ and $d_{\Lambda}(k, l)=1$. It follows that any admissible product $L_{i_{\theta} j_{\theta}} \ldots L_{i_{1} j_{1}}$ of length $\theta \geq n\left(E_{c}, \epsilon, \Lambda\right)$ is positive. By Proposition 1 there must be at least one column such that the sum over this column is less than 1, for some factor $L_{i_{t} j_{t}}$ and hence for the whole product. Therefore the sum over each column of any admissible product of length $2 n\left(E_{c}, \epsilon, \Lambda\right)$ must be less than 1 . Let $c_{0}<1$ be the maximal norm of all admissible products of 
length $2 n\left(E_{c}, \epsilon, \Lambda\right)$. For $k>k_{0}:=\left[\log c / \log c_{0}\right]+1$ we have $c_{0}^{k}<c$ and hence $T=2 k_{0} n\left(E_{c}, \epsilon, \Lambda\right)$ is the required number.

The above argument does not apply to the case $\epsilon=0$, and a different proof must be given for this case (which actually works in general as well). Take $\hat{x} \in \hat{M}$ and let $x(t) \in M$ be the projection of its orbit to $\hat{M}$. Denote $S(x(t))$ by $S_{t}(\hat{x})$, and let

$$
\tilde{S}_{t}(\hat{x})=S_{t}(\hat{x}) \cdots S_{0}(\hat{x}) .
$$

We make the following claims:

1. There exists $\bar{n} \in \mathbb{N}$ such that for all $l, m \in \Lambda$ and all $\hat{x} \in \hat{M}$ there is $t \leq \bar{n}$ such that $\left(\tilde{S}_{t}(\hat{x})\right)_{l m} \neq 0$.

2. For all $i \geq 0$ there exists $n_{i} \in \mathbb{N}$ such that $\left\|C_{m}\left(\tilde{S}_{t}(\hat{x})\right)\right\|_{1}<1$ for all $t \geq n_{i}$, $\hat{x} \in \hat{M}$ and all sites $m \in \Lambda$ with $d_{\Lambda}(m, \partial \Lambda) \leq i$.

The second claim for $i=\frac{1}{2} \operatorname{diam}(\Lambda)$ implies the statement of the theorem.

To see the first claim we fix $\hat{x}$ and let $U \subset \Lambda^{2}$ be the subset of the pairs $(l, m)$ with $\left(\tilde{S}_{t}\right)_{l m}=0$ for all sufficiently large $t$. By inductively applying (2) we see that the columns for $\tilde{S}_{t}(\hat{x})$ are non-zero for all $t \geq 0$. So for all $\beta \in \Lambda$ there is $\alpha \in \Lambda$ such that $(\alpha, \beta) \in \Lambda^{2} \backslash U$. Given sites $\alpha$ and $\beta$ we choose $t$ such that $\tilde{S}_{t}(\hat{x})_{\alpha \beta} \neq 0$. Consider now column $\alpha$ of the matrix $\tilde{S}_{t+1}(\hat{x})=S_{t+1}(\hat{x}) \tilde{S}_{t}(\hat{x})$. If $\alpha$ is stable, i.e. $x(t+1)_{\alpha} \leq E_{c}$, then $\left(S_{t+1}(\hat{x})\right)_{\alpha \alpha}>0$ and $\left(\tilde{S}_{t+1}(\hat{x})\right)_{\alpha \beta} \neq 0$, so we just repeat the argument. But the site $\alpha$ can not be stable for more than $n\left(E_{c}, 0, \Lambda\right)$ iterations. Hence we can with no loss of generality choose $t$ such that $x(t+1)_{\alpha}>E_{c}$. Then the column $\alpha$ of $S_{t+1}(\hat{x})$ has non-zero elements in all position that correspond to neighbors of $\alpha$. Hence we obtain that $\left(\alpha^{\prime}, \beta\right) \in \Lambda^{2} \backslash U$ for all $\alpha^{\prime}$ with $d_{\Lambda}\left(\alpha^{\prime}, \alpha\right)=1$. Any two points can be connected by a path of neighbors, so $U=\emptyset$, and the first claim follows. In fact, one can see that the bound $\bar{n}$ does not depend on a choice of $\hat{x}$ and satisfies: $\bar{n} \leq \operatorname{diam}(\Lambda) \cdot n\left(E_{c}, 0, \Lambda\right)$.

To prove the second claim let us note that if $\left\|C_{k}\left(\tilde{S}_{t}(\hat{x})\right)\right\|_{1}<1$, then $\left\|C_{k}\left(\tilde{S}_{t+1}(\hat{x})\right)\right\|_{1}<1$ because by (2): $\left\|C_{k}(A B)\right\| \leq \max _{l}\left\|C_{l}(A)\right\| \cdot\left\|C_{k}(B)\right\|$.

We will use induction on $i$ starting from $i=0$. Take $k \in \partial \Lambda$ and $t \leq \bar{n}$ such that $\left(\tilde{S}_{t}(\hat{x})\right)_{k k} \neq 0$. If $x(t+1)_{k}>E_{c}$, then $\left\|C_{k}\left(S_{t+1}(\hat{x})\right)\right\|_{1}<1$ and

$$
\left\|C_{k}\left(\tilde{S}_{t+1}(\hat{x})\right)\right\|_{1}=\sum_{l}\left\|C_{l}\left(S_{t+1}(\hat{x})\right)\right\|_{1}\left(\tilde{S}_{t}(\hat{x})\right)_{l k}<1,
$$

and so we have the desired inequality. If $x(t+1)_{k} \leq E_{c}$, then $\left(S_{t+1}(\hat{x})\right)_{k k}=1$ and hence $\left(\tilde{S}_{t+1}(\hat{x})\right)_{k k} \neq 0$. Then we repeat the argument. Since no site can be stable for more than $n\left(E_{c}, 0, \Lambda\right)$ successive time-steps we obtain the claim for $i=0$ with $n_{0}=n\left(E_{c}, 0, \Lambda\right)+\bar{n}$.

Consider now the case $i>0$. For a site $m \in \Lambda$ with $d(m, \partial \Lambda)=i$, we take $l \in \Lambda$ with $d_{\Lambda}(l, m)=1$ and $d_{\Lambda}(l, \partial \Lambda)=i-1$. By the first claim we find some $t \leq \bar{n}$ such that $\left(\tilde{S}_{t}(\hat{x})\right)_{l m} \neq \emptyset$, and by the induction hypothesis for $t^{\prime} \geq n_{i-1}$ we have:

$$
\left\|C_{l}\left(S_{t+t^{\prime}}(\hat{x}) \cdots S_{t+1}(\hat{x})\right)\right\|_{1}<1 .
$$

Using (2) we obtain $\left\|C_{m}\left(\tilde{S}_{t+t^{\prime}}(\hat{x})\right)\right\|_{1}<1$. We can choose $n_{i}=n_{i-1}+\bar{n}$. 
Lemma 6. Let $E_{c} \geq \epsilon /(1-\epsilon)$. Then for any $\hat{x} \in \hat{M}, n \in \mathbb{N}$ and $i, j \in C_{n}(\hat{x})$ we have $d_{\Lambda}(i, j) \neq 1$.

Proof. Take $\hat{x} \in M$ and let $E_{n}$ be the maximal energy of a site in $C_{n}(\hat{x})$. Clearly $E_{1} \leq E_{c}+1$ and

$$
E_{n+1} \leq \max \left\{\max \left\{\epsilon E_{n}, E_{c}\right\}+(1-\epsilon) E_{n}, E_{c}+1\right\} .
$$

From this we see by induction that

$$
E_{n} \leq \max \left\{\frac{E_{c}}{\epsilon}, E_{c}+1\right\}=\frac{E_{c}}{\epsilon},
$$

so $\epsilon E_{n} \leq E_{c}$ for all $n \in \mathbb{N}$, and this means that a site cannot be overcritical in two successive time-steps (for $\epsilon=0$ the above argument does not work, but the statement holds obviously).

All avalanches start with a single site. Let $C_{1}(\hat{x})=\{i\}$. Then $d(i, j)=1$ for all $j \in C_{2}(\hat{x})$. This implies that any two elements of $C_{2}(\hat{x})$ can be connected with a path of length 2 , so no two sites of $C_{2}(\hat{x})$ are nearest neighbors. If there exists a path of even length between two points in $\Lambda$, then all paths connecting these points are of even length. Therefore we can repeat the argument proving by induction that $d_{\Lambda}(i, j) \in 2 \mathbb{Z}$ for all $i, j \in C_{n}(\hat{x})$.

Proposition 7. The linear maps $L_{i j}$ are all invertible whenever $\epsilon \geq 1 / 2$ or $\epsilon>0$ and $E_{c} \geq \epsilon /(1-\epsilon)$. If we have $E_{c} \geq \epsilon /(1-\epsilon)$, then

$$
\operatorname{det} L_{i j}=\epsilon^{s_{i j}} .
$$

Proof. Take arbitrary $x \in \mathbb{R}_{\geq 0}^{N}$. First we observe that since the sum over each column of $Q(x)$ is less than or equal to 1 , we have $\|Q(x) v\|_{1} \leq\|v\|_{1}$ for each $v \in \mathbb{R}^{N}$. This implies that

$$
\begin{aligned}
\|S(x) v\|_{1} & =\|(J(x)+(1-\epsilon) Q(x)) v\|_{1} \\
& \geq\|J(x) v\|_{1}-(1-\epsilon)\|Q(x) v\|_{1} \\
& \geq(2 \epsilon-1)\|v\|_{1} .
\end{aligned}
$$

If $\epsilon>1 / 2$, then $S(x) v \neq 0$ for all $v \neq 0$, so we have invertibility.

For $\epsilon=1 / 2$ the claim follows since in the above chain of inequalities at least one is strict if $v \neq 0$. In fact, if $J(x) v=\epsilon v$, then $v_{i}=0$ for all relaxed sites $i$. We claim that the equality $Q(x) v=v$ is impossible. To see this denote by $\tilde{Q}$ the minor-matrix formed by the rows and columns of $Q(x)$, corresponding to exited sites, and denote by $\tilde{v}$ be the respective reduced vector. Then $\tilde{Q} \tilde{v}=\tilde{v}$.

Let $U$ be the set of overcritical sites $k$ with $v_{k}=\max v_{l}$ (we suppose it is positive, multiplying by -1 in the opposite case). Choose a boundary site $k \in U$, i.e. the number of neighbors $l$ to $k$ with $v_{l}=v_{k}$ is less than $2 d$. Then:

$$
v_{k}=\sum_{l} \tilde{Q}_{k l} v_{l}<v_{k} \sum_{l} \tilde{Q}_{k l} \leq v_{k} .
$$


Dynamics and entropy of SOC

This contradiction yields the result.

Finally consider the last statement about the case $E_{c} \geq \epsilon /(1-\epsilon)$. It is proved by reducing the matrix $S(x)$. If $x_{i} \leq E_{c}$, then column $C_{i}(S(x))$ equals $(0, \ldots, 0,1,0, \ldots 0)^{T}$, where the 1 is in the $i^{\text {th }}$ position. We can start the decomposition of $\operatorname{det} S(x)$ with column $i$, and hence we see that row $i$ and column $i$ can be removed from $S(x)$ without changing the determinant. We remove all rows and columns that correspond to relaxed sites. If $\rho(x)$ is the number of overcritical sites of $x$, we get a $\rho(x) \times \rho(x)$ matrix $S_{\text {red }}(x)$. If site $k$ is overcritical then $J_{k k}(x)=\epsilon$. If $E_{c} \geq \epsilon /(1-\epsilon)$, then it follows from Lemma [ 6 that all nearest neighbors of $k$ are relaxed. Hence column $k$ of $Q(x)$ has only zero entries. This shows that $S_{r e d}(x)=\operatorname{diag}(\epsilon, \ldots, \epsilon)$. Then

$$
\operatorname{det} S(x)=\operatorname{det} S_{r e d}(x)=\epsilon^{\rho(x)},
$$

so $\operatorname{det} L_{i j}=\epsilon^{s_{i j}}$.

Remark 2. In the original model of Zhang one has $\epsilon=0$ in which case $\operatorname{det} L_{i j}=$ 0 if $L_{i j} \neq 1$. But it is not true that non-trivial kernels can occur for $\epsilon=0$ only, contrary to what was stated in $[B C K]$. A simple counter-example is the case $N=2, E_{c}=1 / 3$ and $\epsilon=1 / 3$. For $x_{1}>0$ and $2 x_{1}+3 x_{2}<1$ we have:

$$
F_{1}\left(\left[\begin{array}{l}
x_{1} \\
x_{2}
\end{array}\right]\right)=\frac{1}{9}\left[\begin{array}{ll}
2 & 3 \\
2 & 3
\end{array}\right]\left[\begin{array}{c}
x_{1}+1 \\
x_{2}
\end{array}\right] \text {. }
$$

and so $\operatorname{det} L_{12}=0$.

Having non-degenerate maps in the model is more convenient from the point of view of mathematical tools (though from a physical viewpoint it can make no big difference between degenerate and close-to-degenerate systems). Fortunately, degenerations occur only for a negligible set of parameters.

Theorem 8. The maps $L_{i j}$ are invertible for almost all $\left(\epsilon, E_{c}\right)$. In fact, they are invertible for the parameters complimentary to the set $\Xi \subset[0,1) \times(0, \infty)$, which consists of a finite set of vertical intervals for fixed $d$ and $N$.

Proof. Fix an avalanche $A_{i j}$ and let $L_{i j}^{\epsilon}$ be the corresponding linear maps (we stress dependence on $\epsilon$ ). These maps are the compositions of elementary matrices $S^{\epsilon}(x(\tau(x, i))) \ldots S^{\epsilon}(x(1))$, with the factors from (11)

$$
S^{\epsilon}(x(t))=\mathbf{1}+(\epsilon-1)\left(\frac{d J}{d \epsilon}-Q\right)(x(t))
$$

being polynomial in $\epsilon$ and independent of the choice of $x=x(1) \in M_{i j}$. The condition $\operatorname{det} L_{i j}^{\epsilon}=0$ is equivalent to $\operatorname{det} S^{\epsilon}(x(t))=0$ for some $t$. Denoting by $\mathrm{Sp}_{-}(T)$ the negative part of the spectrum of $T$, we get: $\epsilon \in 1+\mathrm{Sp}_{-}\left(Q-\frac{d J}{d \epsilon}\right)^{-1}$.

There are only finite number of possibilities for the matrix $S^{\epsilon}(x)$ (though a countable number for their compositions $L_{i j}^{\epsilon}$, the length of which grow as $E_{c} \rightarrow$ $0)$. Thus we obtain $k=k(d, N)$ different values of $\epsilon$ for which $\operatorname{det} S^{\epsilon}(x)=0$ : $\left\{\epsilon_{a}\right\}_{a=1}^{k}$. For each $\epsilon_{a}$ there is the maximal value $E_{c}^{a}$ of $E_{c}$ (finite if $\epsilon_{a} \neq 0$ ), where the corresponding matrix $S^{\epsilon}(x)$ can appear in the avalanche. Thus the set of degenerate systems is $\left\{\left(\epsilon, E_{c}\right) \mid \epsilon=\epsilon_{a}, 0<E_{c}<E_{c}^{a}\right\}$.

By proposition $\left[7 \Xi\right.$ does not intersect the set $\{\epsilon \geq 1 / 2\} \cup\left\{E_{c} \geq \epsilon /(1-\epsilon)\right\}$. 
Boris Kruglikov, Martin Rypdal

\section{Removability of singularities and coding}

The map $F$ may be considered as a piecewise affine map $F: I \times M \rightarrow I \times M$, where $I=[0,1]$ and $F(t, x)=\left(N t \bmod 1, F_{[N t]}(x)\right)$. The map $t \mapsto N t \bmod 1$ is not conjugated to $\sigma_{N}^{+}$since the points $m / N^{k} \in I$ do not have unique representations in $\Sigma_{N}^{+}$. However the sets $\left\{m / N^{k}\right\} \times M \subset I \times M$ are singularities, and following the standard approach for piecewise affine maps, should be removed.

In some physical systems, like the Belykh family, the singularities propagate, intersecting themselves transversally. The Zhang model is not a general position system in this respect, because singularities $\left\{m / N^{k}\right\} \times M \subset I \times M=\hat{M}$ map into themselves, forming zero angle.

\subsection{Construction of attractors}

Define the (spatial) singularity set $S(F)=\cup_{i j} \partial M_{i j}$. Then $U=M \backslash S(F)$ consists of a collection of open connected sets $\mathcal{Z}=\{Z\}$. Let $U_{0}:=U$ and

$$
U_{n}:=\bigcup_{i \in \Lambda} F_{i}\left(U_{n-1}\right) \cap U
$$

We say that $x \in S(F)$ is a non-essential singularity of order $m$ if there exists $\epsilon>0$ and $m>0$ such that

$$
\operatorname{card}\left\{Z \in \mathcal{Z} \mid U_{n} \cap B_{\epsilon}(x) \cap Z \neq \emptyset\right\} \leq 1
$$

for all $n>m$. Denote the set of non-essential singularities of order $m$ by $N E S(F ; m)$ and let $N E S(F):=\cup_{m \geq 0} N E S(F ; m)$ be the set of all non-essential singularities. Define $E S(F)=S(F) \backslash N E S(F)$ to be the collection of essential singularities. Observe that there is a natural extension of $F$ to $V_{0}=U \cup$ $N E S(F)$. In the following we let $F$ denote the extended map. As above we define

$$
V_{n}:=\bigcup_{i \in \Lambda} F_{i}\left(V_{n-1}\right) \cap V_{0} .
$$

Let $\mathcal{X}=\cap_{n \geq 0} V_{n}$ and $\mathcal{D}=\Sigma_{N}^{+} \times \mathcal{X}$. Clearly $F(\mathcal{D})=\mathcal{D}$. The set $\mathcal{Y}=\overline{\mathcal{X}}$ is called the physical (or spatial) attractor of $F$, and $\mathcal{A}=\Sigma_{N}^{+} \times \mathcal{Y}=\overline{\mathcal{D}}$ is the extended attractor of $F$.

Proposition 9. $\left.F\right|_{\mathcal{D}}$ is continuous.

Proof. The set $\mathcal{D}$ intersects non-essential singularities only. Hence we must show that if $x$ is a non-essential singularity in $\mathcal{D}$, then the extension of each $F_{i}$ to $N E S(F)$ is continuous at the point $x$. Choose $m \in \mathbb{N}$ and $\varepsilon>0$ such that $B_{\varepsilon}(x) \cap U_{n}$ intersects only one partition element $Z \in \mathcal{Z}$ for $n>m$ and let $y \in B_{\varepsilon / 2}(x) \cap \mathcal{D}$. Then $B_{\varepsilon / 2}(y) \subset B_{\varepsilon}(x)$ and so $B_{\varepsilon / 2}(y) \cap U_{n}$ intersects the same partition element $Z$. So $x$ and $y$ are mapped by the same affine map $\left.F_{i}\right|_{Z}$ for each $i \in \Lambda$. The claim follows. 
Dynamics and entropy of SOC

In general, the map $F$ does not have a continuous extension to $\mathcal{A}$, but only to $\mathcal{A} \backslash\left(\Sigma_{N}^{+} \times E S(F)\right)$. Actually, if $x \in E S(F) \cap \mathcal{Y}$ lies on the boundary of several continuity partitions for $F_{i}$, then there are several extensions of $F$ to $(i, x)$. Thus we can continuously extend $F$ to $\mathcal{A}$ only when the essential singularities do not intersect the attractor (are removable).

\subsection{Symbolic Coding}

If the singularities can affect the dynamics only for a finite number of iterations, then the dynamics can be well approximated by a topological Markov chain.

Definition 2. We say that singularities are removable if there exists $m \in \mathbb{N}$ such that $S(F)=N E S(F, m)$.

The physically most relevant observables $\phi: \hat{M} \rightarrow \mathbb{R}$ are those that are determined by avalanches. We say that $\phi$ is an avalanche observable if it is constant on continuity domains $[i] \times M_{i j}$.

Theorem 10. If singularities are removable, then the map $F$ is well-defined and continuous on $\mathcal{A}$ and there is a topological Markov-chain $\left(\Sigma_{A}^{+}, \sigma_{A}^{+}\right)$and a continuous semi-conjugancy $g: \mathcal{A} \rightarrow \Sigma_{A}^{+}$such that for all $\hat{x}, \hat{y} \in \mathcal{A}$ and for all avalanche observables $\phi$ we have:

$$
g(\hat{x})=g(\hat{y}) \Rightarrow \phi\left(F^{n}(\hat{x})\right)=\phi\left(F^{n}(\hat{y})\right) \forall n \geq 0 .
$$

The Markov-chain is determined by a matrix A which has a maximal eigenvalue equal to $N$.

Remark 3. It is clear that all properties related to distribution of avalanche size, duration, area, etc. are invariant under a semi-conjugancy such as this. Observe that for each avalanche observable $\phi$ on $\mathcal{A}$, there is a unique observable $\phi^{\prime}: \Sigma_{A}^{+} \rightarrow \mathbb{R}$ such that $\phi=\phi^{\prime} \circ \mathrm{g}$. Suppose we have a measure $\mu$ on $\mathcal{A}$, and let $\nu=g_{*} \mu$. If $\phi$ is an avalanche observable on $\mathcal{A}$, then the statistical properties of $\phi$ with respect to $\mu$ are equivalent to the statistical properties of $\phi^{\prime}$ with respect to $\nu$. In this $\left(\sigma_{A}^{+}, \Sigma_{A}^{+}\right)$is a good approximation to $\left.F\right|_{\mathcal{A}}$. The coding gives estimates on entropy and growth of periodic points, but these estimates are asymptotically no better than what we get from the trivial semi-conjugancy $\hat{M} \rightarrow \Sigma_{N}^{+}$.

Proof. Singularities are removable so there exists an integer $m \in \mathbb{N}$ such that $\pi_{s} \circ F^{m}(\hat{M})$ only intersects trivial singularities. Let $X_{1}, \ldots, X_{s}$ be the closure of the connected components of $\pi_{s} \circ F^{m}(\hat{M}) . \quad F$ is well defined and continuous on these components. Let $Y_{1}, \ldots, Y_{s}$ be the intersections of the components $X_{1}, \ldots, X_{s}$ with $\mathcal{Y}$. We construct the partition $\mathcal{R}=\left\{[i] \times Y_{k}\right\}$ and enumerate it so that $\mathcal{R}=\left\{R_{1}, \ldots, R_{r}\right\}$, where $r=N s$.

Let $A=\left\|a_{i j}\right\|$ be the $r \times r$ matrix defined by the rule: $a_{i j}=1$ if $F\left(R_{i}\right) \cap R_{j} \neq$ $\emptyset$, and $a_{i j}=0$ otherwise. A sequence $R_{\omega_{0}} R_{\omega_{1}} \ldots$ is legal if $a_{\omega_{t-1} \omega_{t}}=1$ for all $t \in \mathbb{N}$. Define $g: \mathcal{A} \rightarrow \Sigma_{A}^{+}$by $g(\hat{x})=\left(\omega_{0} \omega_{1} \ldots \omega_{t} \ldots\right)$, where $F^{t}(\hat{x}) \in R_{\omega_{t}}$. 


\section{Boris Kruglikov, Martin Rypdal}

To prove that $g$ is surjective it suffices to show that for each legal sequence $R_{\omega_{0}} R_{\omega_{1}} \ldots$, there is a point $\hat{x} \in \pi_{s} \circ F^{m}(\hat{M})$ such that $F^{i}(\hat{x}) \in R_{\omega_{t}}$ for all $i \in \mathbb{N}$. Note that each $\omega$ can be written as a pair $(t, k)$, where $t \in\{1, \ldots, N\}$ and $k \in\{1, \ldots, s\}$. Hence we can write

$$
\bigcap_{n=0}^{\infty} F^{-n}\left(R_{\omega_{n}}\right)=\bigcap_{n=0}^{\infty} F^{-n}\left(\left[t_{n}\right] \times Y_{k_{n}}\right)=\{\mathbf{t}\} \times \bigcap_{n=0}^{\infty} F_{t_{0}}^{-1} \circ \cdots \circ F_{t_{n-1}}^{-1}\left(Y_{k_{n}}\right) .
$$

The continuous image of a connected set is connected, so for each $i=1, \ldots, N$ and each $k=1, \ldots, s$ there is a unique $l \in\{1, \ldots, s\}$ such that $F_{i}\left(X_{k}\right) \subset X_{l}$. This implies that we have a nested sequence

$$
Y_{0} \subset F_{t_{0}}^{-1}\left(Y_{k_{1}}\right) \subset F_{t_{0}}^{-1} \circ F_{t_{1}}^{-1}\left(Y_{k_{1}}\right) \subset \ldots
$$

and hence the intersection is non-empty.

It is clear that $g_{\mathcal{R}}$ is continuous (see $[\mathrm{R}$ for details). Since the partition $\mathcal{R}$ is a refinement of the continuity partition the conjugancy will be injective up to the classes of points that follow the same continuity domains. Hence if $\phi\left(F^{n} \hat{x}\right) \neq \phi\left(F^{n} \hat{y}\right)$ for some avalanche observable $\phi$ and some $n \geq 0$, then $g(\hat{x}) \neq g(\hat{y})$.

Remark 4. Suppose we modify the Zhang model by using a full shift $\left(\Sigma_{N}, \sigma_{N}\right)$ as the excitation factor. It is then possible that the modified map $F$ is injective on $\Sigma_{N} \times \mathcal{Y}$. Since we have strict attraction in the spatial factor after a fixed number of iterations it is clear that we can then obtain an injective coding, and hence a topological conjugancy. However, if we make this modification it is not clear that $\Sigma_{N} \times \mathcal{Y}$ equals the set

$$
\Omega=\overline{\bigcap_{n=-\infty}^{\infty} F^{n}\left(\Sigma_{N} \times M\right)} .
$$

In fact if the maps $\left.F_{i}\right|_{\mathcal{Y}}$ are all injective, then $\left.F\right|_{\Omega}$ is invertible, but $\left.F\right|_{\Sigma_{N} \times \mathcal{Y}}$ is typically non-invertible. The reason for this is that, due to contraction, a point $x \in \mathcal{Y}$ does not have preimages for all the maps $F_{i}$ and $F_{i}$ are invertible only on $F_{i}(\mathcal{Y}) \subset \mathcal{Y}$. So to obtain invertibility we must turn to the attractor $\Omega$. From a physical point of view the spatial attractor is of the great interest, so it is desirable to have an attractor which is a Cartesian product of the Bernoulli shift and the spatial attractor $\mathcal{Y}$.

We can always construct a coding of $\left.F\right|_{\mathcal{D}}$ (even in non-removable case) by choosing a partition $\mathcal{R}=\left\{R_{1}, \ldots R_{r}\right\}$, and taking $g_{\mathcal{R}}: \mathcal{D} \rightarrow\{1, \ldots, r\}^{\mathbb{N}}$ to be the map sending a point $\hat{x} \in \mathcal{D}$ to the unique sequence $\omega \in\{1, \ldots r\}^{\mathbb{N}}$ such that $F^{t}(\hat{x}) \in R_{\omega_{t}}$ for all $t \geq 0$. But there is no reason, however, to expect $g_{\mathcal{R}}(\mathcal{D})$ to be a topological Markov chain, cf. [BCK]. 
Dynamics and entropy of SOC

\section{Metric properties}

The natural volume on $\hat{M}$ is given by the product measure of the uniform Bernoulli measure on $\Sigma_{N}^{+}$and the Lebesgue measure on $M$. By iterating this measure (and averaging) we can construct SRB-measures. However it can happen that the measures constructed are supported on essential singularities, where it is not possible to define the dynamics in such a way that the measure is invariant. Hence we must give some conditions to ensure the existence of SRB-measures. If there is an SRB-measure it is characterized by the fact that its projection to $\Sigma_{N}^{+}$coincides with the uniform Bernoulli measure. From this it follows that any SRB-measure is a measure of maximal entropy. In situations where the system allows symbolic coding the SRB-measure corresponds to the Perry measure on the topological Markov chain $\Sigma_{A}^{+}$.

\subsection{Existence and characterization of SRB-measures}

Let $m=m^{u} \times m^{s}$, where $m^{u}=\mu_{\text {Ber }}$ is the uniform Bernoulli measure on $\Sigma_{N}^{+}$, and $m^{s}=\mu_{\text {Leb }}$ is the Lebesgue measure on $M$. We say that an invariant Borel probability measure $\mu$ on $\hat{M}$ has the SRB-property if there exists a measurable invariant set $G \subset \hat{M}$ such that

1. $m(G)>0$

2. $m^{u}\left(\pi_{u}(G)\right)=1$

3. All points $\hat{x} \in G$ are future generic with respect to $\mu$, i.e.

$$
\frac{1}{n} \sum_{t=0}^{n-1} \phi\left(F^{t} \hat{x}\right) \rightarrow \int \phi d \mu,
$$

for all $\hat{x} \in G$ and all continuous functions $\phi: \hat{M} \rightarrow \mathbb{R}$.

For Axiom A attractors one can ensure the existence of measures for which the set of generic points has full Lebesgue measure and this is equivalent to saying that the canonical family of conditional measures on the unstable manifolds are absolutely continuous with respect to the Lebesgue measure. For non-invertible maps one can in general only expect the set of generic points to have positive measure and hence it is unreasonable to require that $m(G)=1$. Condition 2 is (for physical reasons) important in the Zhang model. It means that the statistical properties do not depend on the choice of a generic sequence $\mathbf{t}$ of excitations. (This is always implicitly assumed in the numerical investigations of the Zhang model that can be found in the physical literature.) Moreover, condition 2 will be satisfied for the SRB-measures that can be constructed by iterating the measure $m$.

By a standard approach we can give conditions for existence of SRB-measures that hold if singularities are removable, but it is not known if these conditions hold in all non-removable situations. 


\section{Boris Kruglikov, Martin Rypdal}

Proposition 11. Let $\left(\epsilon, E_{c}\right)$ does not belong to the negligible set $\Xi$ of Theorem 8. If there exists $n \geq 0, C>0$ and $q>0$ such that

$$
\forall \delta>0, \forall t \geq 0: m\left(F^{-t}\left(\Sigma_{N}^{+} \times U_{\delta}(E S(F ; n))\right) \leq C \delta^{q},\right.
$$

then there exists a set $\mathcal{D} \subset \hat{M}$ (constructed in 2.1 ), which may intersect singularities, and a natural extension of $F$ to $\mathcal{D}$ such that $F(\mathcal{D})=\mathcal{D}$. Moreover the set $\mathcal{D}$ carries an $F$-invariant Borel probability measure with the SRB-property.

Remark 5. Proposition 11 is a simple modification of the result of Schmeling and Troubetzkoy $[S T]$. In their paper the conditions for existence are in general too restrictive for the Zhang model. In fact, in Example $A$ of $\sqrt{6}$ we show a situation where the SRB-measure constructed in [ST] does not exist, but we clearly have existence of a physically relevant measure. The reason for this paradox is that one in general remove all singular points on the construction of the attractor, even if there is a natural extension of $F$ to the points of singularity. (Proposition [1] obviously applies to this example since $E S(F ; 5)=\emptyset$.)

Proof. In $[\mathrm{ST}]$ it is shown that a piecewise smooth map $f$ with singularity set $S$ has a measure, not supported on singularities, such that the set of generic points has positive Lebesgue measure. They require that the following conditions are satisfied:

1. The restrictions of $f$ to each of its continuity domains are diffeomorphisms onto their image.

2. The second differentials $D^{2} f_{x}$ does not grow too fast close to singularities. (See [ST] for a more precise formulation.)

3. $f$ is hyperbolic. In this context this means that there are constants $C>0$ and $\lambda \in(0,1)$ such that for all $x \notin S$ there is a splitting of the tangent space at $x$ into subspaces $E^{+}(x)$ and $E^{-}(x)$. There are cones $C^{+}(x)$ and $C^{-}(x)$ around $E^{+}(x)$ and $E^{-}(x)$ that are invariant under $D f_{x}$ and $D f_{x}^{-1}$ respectively. The angles between the $C^{+}(x)$ and $C^{-}(x)$ are bounded away from zero, and for all points $x$ that do not intersect singularities in the first $n$ iterations it holds:

$$
\left\|D_{x} f^{n}(v)\right\| \geq C^{-1} \lambda^{-n}\|v\| \text { for } v \in C^{+}(x),
$$

and

$$
\left\|D_{x} f^{n}(v)\right\| \leq C \lambda^{n}\|v\| \text { for } v \in C^{-}(x) .
$$

4. There exists $C>0$ and $q>0$ such that $m\left(f^{-t}\left(U_{\varepsilon}(S)\right)\right) \leq C \varepsilon^{q}$ for all $\varepsilon>0$ and all $t \in \mathbb{N}$.

We apply this result to the map $\left.F\right|_{\Sigma_{N}^{+} \times\left(U_{n} \backslash E S(F ; n)\right)}$. The singularity set for this map is contained in $\Sigma_{N}^{+} \times E S(F)$, so by assumption condition 4 is satisfied. Condition 1 follows from Proposition [7 condition two is obviously satisfied since 
$F$ is piecewise affine and condition 3 follows from Theorem 5 with $E^{+}=\mathbb{R}^{1} \oplus 0$, $E^{-}=0 \oplus \mathbb{R}^{N}$ and $C^{ \pm}$being the regular cones around them (actually Theorem 5 ensures hyperbolicity for some iterate $F^{T}$, which implies the claim).

In [ST] the measures are constructed by iterating $m$, averaging and taking a weak limit. It is clear that, in the Zhang model, any measure obtained in this way will satisfy condition 2 in our definition of an SRB-measure.

If an SRB-measure exists it can be characterized by a number of different properties. From a physical perspective it is reasonable to require that a relevant invariant measure should preserve the uniform Bernoulli structure on $\Sigma_{N}^{+}$. This corresponds to the Lebesgue measure on $[0,1]$ in the alternative formulation of the map $F$, and hence to absolutely continuous measure conditional measures on the unstable space $[0,1]$.

Proposition 12. If $\mu$ is an SRB-measure on $\mathcal{D}$, then $\mu^{u}:=\left(\pi_{u}\right)_{*} \mu$ is the uniform Bernoulli measure on $\Sigma_{N}^{+}$.

Proof. There is a set $A=\pi_{u}(G)$ of full $m^{u}$-measure, such that all $\mathbf{t} \in A$ are generic with respect to $\mu^{u}$. Take a continuous function $\phi: \Sigma_{N}^{+} \rightarrow \mathbb{R}$. Then

$$
\int \phi d \mu^{u}=\lim _{n \rightarrow \infty} \frac{1}{n} \sum_{t=0}^{n-1} \phi\left(\left(\sigma_{\Lambda}^{+}\right)^{t} \mathbf{t}\right)=\int \phi d m^{u}
$$

where the left equality holds for $\mathbf{t} \in A$ and the right one for $\mathbf{t} \in B$ with $B \subset \Sigma_{N}^{+}$ a subset of full $m^{u}$-measure (from Birkhoff ergodic theorem). Since $A \cap B \neq \emptyset$, we get: $\int \phi d \mu^{u}=\int \phi d m^{u}$ for all continuous functions $\phi$.

\subsection{Measures of maximal entropy}

Suppose that there exists an invariant Borel probability measure $\mu$ on $\hat{M}$. Let $\mu^{u}:=\left(\pi_{u}\right)_{*} \mu$ and let $\left\{\nu_{\mathbf{t}}\right\}$ to be the canonical family of conditional measures on the fibers $\pi_{u}^{-1}(\{\mathbf{t}\})$. By the Abramov-Rokhlin formula

$$
h_{\mu}(F)=h_{\mu^{u}}\left(\sigma_{N}^{+}\right)+h_{\mu}\left(F \mid \sigma_{N}^{+}\right)
$$

where

$$
h_{\mu}\left(F \mid \sigma_{N}^{+} ; \mathcal{Q}\right)=\lim _{n \rightarrow \infty} \frac{1}{n} \int H_{\nu_{\mathbf{t}}}\left(\bigvee_{k=0}^{n-1}\left(F_{t_{k-1}} \circ \cdots \circ F_{t_{0}}\right)^{-1}(\mathcal{Q})\right) d \mu^{u}(\mathbf{t}),
$$

for a partition $\mathcal{Q}$ and

$$
h_{\mu}\left(F \mid \sigma_{N}^{+}\right)=\sup _{\mathcal{Q}} h_{\mu}\left(F \mid \sigma_{N}^{+} ; \mathcal{Q}\right),
$$

The supremum is taken over all finite measurable partitions $\mathcal{Q}$ of $\hat{M}$. This formula was originally proved for product measures by Abramov and Rokhlin [AR and extended to arbitrary skew products by Bogenschultz and Crauel [BC].

Below we use the notation $F_{\mathbf{t}}$ for the dynamics over a pre-fixed sequence $\mathbf{t}=\left(t_{0} t_{1} \ldots\right) \in \Sigma_{N}^{+}$. By $n$-the iteration we mean the map $F_{\mathbf{t}}^{n}=F_{t_{n-1}} \circ \cdots \circ F_{t_{0}}$. 
Theorem 13. If $\mu$ is an invariant Borel probability measure on $\hat{M}$, then $h_{\mu}(F)=$ $h_{\mu^{u}}\left(\sigma_{N}^{+}\right)$.

Proof. We prove the proposition by estimating $h_{\mu}\left(F \mid \sigma_{N}^{+}\right)$from above. Let $\mathcal{Q}$ be a partition of $M$ and

$$
\mathcal{Q}_{t_{0} \ldots t_{n-1}}:=\bigvee_{k=0}^{n-1}\left(F_{t_{k-1}} \circ \cdots \circ F_{t_{0}}\right)^{-1}(\mathcal{Q})=\bigvee_{k=0}^{n-1} F_{\mathbf{t}}^{-k}(\mathcal{Q})
$$

Fix $\varepsilon>0$ and choose the partition $\mathcal{Q}$ such that $h_{\mu}\left(F \mid \sigma_{N}^{+} ; \mathcal{Q}\right)+\varepsilon \geq h_{\mu}\left(F \mid \sigma_{N}^{+}\right)$. The maps $F_{i}$ are non-expanding so it follows from the Ruelle-Margulis inequality [KH] that

$$
\frac{1}{n} H_{\nu_{\mathbf{t}}}\left(\bigvee_{k=0}^{n-1} F_{\mathbf{t}}^{-k}(\mathcal{Q})\right) \longrightarrow h_{\nu_{\mathbf{t}}}\left(F_{\mathbf{t}}\right)=0
$$

Moreover the convergence is $\mu^{u}$-uniform and so the same holds for the integrals. Another way to see it is via the multiplicity notion of 4.2 (then $\mathcal{Q}$ should be subordinate to each continuity partition $\left.\left\{M_{i j} \mid j=1, \ldots, q_{i}\right\}\right)$ :

$$
h_{\mu}\left(F \mid \sigma_{N}^{+} ; \mathcal{Q}\right) \leq \lim _{n \rightarrow \infty} \frac{1}{n} \log \max _{|\mathbf{t}| \leq n} \operatorname{mult}\left(\mathcal{Q}_{t_{0} \ldots t_{n-1}} \cap \operatorname{supp}\left(\nu_{\mathbf{t}}\right)\right)
$$

Therefore $h_{\mu}\left(F \mid \sigma_{N}^{+}\right) \leq \epsilon$. Let $\epsilon \rightarrow 0$.

Remark 6. Theorem $[13$ is a partial case of Theorem 4 from [KR2].

We say that an invariant measure $\mu$ is maximal if $h_{\mu}(F)=\sup _{\nu} h_{\nu}(F)$, where the supremum is taken over all invariant Borel probability measures on $M$. It follows from Theorem 13 that $h_{\mu}(F) \leq \log N$. So if $h_{\text {top }}(F)>\log N$, the variational principle fails (this can happen for piece-wise affine systems, see [KR1, KR2]). But we show in 4.2 that the abnormal growth of $h_{\text {top }}(F)$ does not occur in the Zhang model, at least for generic values of parameters $E_{c}, \epsilon$.

Corollary 1. Any SRB-measure on $\mathcal{D}$ has entropy $h_{\mu}(F)=\log N$ and is hence a maximal measure.

Corollary 2. Suppose singularities are removable and that $\mu$ is an SRB-measure on $\mathcal{A}$. Let $g: \mathcal{A} \rightarrow \Sigma_{A}^{+}$be the semi-conjugacy constructed in the proof of Theorem 10. If $\left(\sigma_{A}^{+}, \Sigma_{A}^{+}\right)$is topologically transitive, then $g_{*} \mu$ is the Perry measure on $\Sigma_{A}^{+}$.

Proof. A transitive topological Markov chain has a unique measure of maximal entropy. This measure is called the Perry measure $[\mathrm{KH}]$. 
Dynamics and entropy of SOC

\subsection{Hyperbolic structure}

There are several ways to define Lyapunov exponents for the Zhang model. The Zhang model can be represented as a piecewise affine map, where Bernoulli shift is represented as the expanding map $t \mapsto N t \bmod 1$ of the interval (see 4.2 ). Hence it is clear that there is one positive Lyapunov exponent $\chi_{0}^{+}=\log N$. We define the other exponents by introducing the co-cycle $\mathcal{T}: \hat{M} \rightarrow G L(N, \mathbb{R})$, defined by $\mathcal{T}(\hat{x})=L_{i j}$, where $\hat{x} \in[i] \times M_{i j}$. For $\hat{x} \in \hat{M}$ and $v \in \mathbb{R}^{N} \backslash\{0\}$ we define

$$
\chi(\hat{x}, v)=\varlimsup_{n \rightarrow \infty} \frac{1}{n} \log \frac{\left\|\mathcal{T}\left(F^{n-1}(\hat{x})\right) \ldots \mathcal{T}(F(\hat{x})) \mathcal{T}(\hat{x}) v\right\|}{\|v\|} .
$$

It is a general fact that the function $\chi(\hat{x}, \cdot)$ takes at most $N$ different values $\chi_{1}^{-}(\hat{x}) \geq \cdots \geq \chi_{N}^{-}(\hat{x})$.

Proposition 14. For all $\hat{x} \in \hat{M}$ the Lyapunov spectrum is:

$$
\chi_{0}^{+}=\log N>0>\chi_{1}^{-}(\hat{x}) \geq \cdots \geq \chi_{N}^{-}(\hat{x})
$$

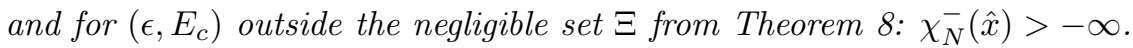

Proof. From Theorem $[5$ we know that there exists $T \in \mathbb{N}$ and $c \in(0,1)$ such that

$$
\left\|\mathcal{T}\left(F^{T-1}(\hat{x})\right) \ldots \mathcal{T}(F(\hat{x})) \mathcal{T}(\hat{x})\right\| \leq c
$$

for all $\hat{x} \in \hat{M}$. It immediately follows that $\chi(\hat{x}, v) \leq T^{-1} \log c<0$.

For $\epsilon \geq 1 / 2$ and arbitrary $E_{c}$ or for $\epsilon>0$ and $E_{c} \geq(1+\epsilon) /(1-\epsilon)$ all linear maps are invertible, and so for all $\hat{x} \in \hat{M}$ and all $v \in \mathbb{R}^{N} \backslash\{0\}$ we have $\chi(\hat{x}, v) \geq \log k$, where $k=\min _{i j} \min \operatorname{Sp}\left(L_{i j}\right)>0$.

If there exists a unique SRB-measure, then it follows from the Osceledec theorem that there are numbers $\chi_{1}^{-}, \ldots, \chi_{N}^{-}$such that $\chi_{i}(\hat{x})=\chi_{i}^{-}$for Lebesgue almost every $\hat{x} \in \hat{M}$. The numbers $\chi_{0}^{+}, \chi_{1}^{-}, \ldots, \chi_{N}^{-}$are the Lyapunov exponents of the Zhang model. From Proposition [14] it follows that the Zhang model is hyperbolic in the sense that the Lyapunov spectrum consists of:

$$
\chi_{0}^{+}=\log N>0>\chi_{1}^{-} \geq \cdots \geq \chi_{N}^{-}>-\infty .
$$

We see from Corollary 1 that the Pesin formula $h_{\mu}(F)=\chi^{+}$holds for any SRBmeasure. If there is no SRB-measure then the Lyapunov spectrum should be defined as functions on $M$ :

$$
\chi_{i}(x)=\int_{\Sigma_{N}^{+}} \chi_{i}(\mathbf{t}, x) d \mu_{\mathrm{Ber}},
$$

where $\mu_{\text {Ber }}$ is the uniform Bernoulli measure on $\Sigma_{N}^{+}$. 


\subsection{Entropy of physical vs. mathematical models}

We can reformulate the Zhang system as the map $\hat{f}: \hat{B} \rightarrow \hat{B}$, where $\hat{B}=$ $\cup_{i \geq 0} \hat{f}^{i}(\hat{M})$ is a compact $\hat{f}$-invariant subset of $\Sigma_{N}^{+} \times \mathbb{R}^{N}$. We wish to compare this to the induced transformation $F: \hat{M} \rightarrow \hat{M}$ (cf. Remark 1).

If $\mu$ is an $F$-invariant Borel probability measure on $\hat{M}$, then there is an associated $\hat{f}$-invariant Borel probability measure $\hat{\mu}$ on $\hat{B}$ (and vice versa). Abramov's theorem $([\mathrm{Br}])$ relates the entropies of both systems:

$$
h_{\hat{\mu}}(\hat{f})=h_{\mu}(F) \cdot \hat{\mu}(\hat{M}) .
$$

One does not need to assume ergodicity and can allow degenerations [DGS, as happens for the case of Zhang model. In ergodic situation by the recurrence theorem of $\mathrm{Kac}[\mathrm{Br}]$ for a $\mu$-generic point $\hat{x} \in \hat{M}$ :

$$
\frac{1}{\hat{\mu}(\hat{M})}=\lim _{n \rightarrow \infty} \frac{1}{n} \sum_{k=0}^{n-1} \tau\left(F^{k} \hat{x}\right),
$$

where $\tau(\hat{x})=\tau(i, x)$ is the avalanche time initiated by addition of $e_{i}$ to $x \in M$ (see 1.2). If $\mu=\mu_{\mathrm{SRB}}$ is a unique SRB-measure, the above point $\hat{x}$ can be chosen Lebesgue generic. The resulting limit is the average avalanche time $\bar{\tau}$ (we discuss it in more details in $\$ 7.17 .2$ ) and we obtain:

$$
h_{\hat{\mu}}(\hat{f})=h_{\mu}(F) / \bar{\tau} \quad \text { resp. } \quad h_{\hat{\mu}_{\mathrm{SRB}}}(\hat{f})=h_{\mu^{u}}\left(\sigma_{N}^{+}\right) / \bar{\tau} .
$$

In general non-ergodic situation to get equality (4) we should integrate the terms in right-hand side and then we again obtain the average avalanche size $\langle\tau\rangle$, but now it is the space-average. Substituting this into (3) we get:

$$
h_{\hat{\mu}}(\hat{f})=h_{\mu}(F) /\langle\tau\rangle \text {. }
$$

For SRB-measures this formula is indicated by the Ledrappier-Young theorem [LY], because we have only one positive Lyapunov exponent.

\section{Topological entropy}

To calculate the topological entropy of $F$ we established in KR2 a set of inequalities, using the technique developed by J. Buzzi [B1], B2] for piecewise expanding maps and piecewise isometries, see Appendix The contraction in the maps $F_{i j}$ provides difficulties, so several results were generalized to fit the framework of the Zhang model. It is not however true (as was widely believed, see [B2]) that the contraction does not contribute to topological (contrary to metric) entropy, the corresponding counter-example can be found in KR1. The Zhang model has a feature common to all such examples KR2, namely angular expansion, but still for most values of the parameters this abnormal increase of the entropy does not occur. 
Dynamics and entropy of SOC

\subsection{Growth of the number of continuity domains}

Let $\mathcal{P}=\left\{[i] \times M_{i j}\right\}$ be the partition of continuity for $F$, and enumerate the elements so that $\mathcal{P}=\left\{P_{1}, \ldots P_{r}\right\}$. Let

$$
\left[P_{a_{0}} \ldots P_{a_{n-1}}\right]:=\bigcap_{m=0}^{n-1} F^{-m}\left(P_{a_{m}}\right),
$$

and

$$
\mathcal{P}^{n}=\left\{\left[P_{a_{0}} \ldots P_{a_{n-1}}\right] \neq \emptyset \mid a_{m}=1, \ldots, r\right\} .
$$

We define the singularity entropy of $F$ by

$$
H_{\text {sing }}(F)=\lim _{n \rightarrow \infty} \frac{1}{n} \log \operatorname{card}\left(\mathcal{P}^{n}\right) .
$$

Remark 7. Define a map $g: \mathcal{A} \rightarrow \Sigma_{r}^{+}$by letting $g(\hat{x})$ be the unique sequence $a_{0} a_{1} \ldots$ such that $F^{n}(\hat{x}) \in P_{a_{n}}$. Then

$$
H_{\text {sing }}(F)=h_{\text {top }}\left(\sigma_{r}^{+} ; g(\mathcal{A})\right) .
$$

For piecewise affine expanding maps and piecewise isometries it is clear that this also equals the topological entropy, but due to the contraction, this is not obvious in the Zhang model. In addition, if singularities are not removable, the map $g$ has discontinuities.

Definition 3. Call a point $x \in S(F)$ an unstable singularity if for all $i$ and $k \neq l$ we have: $\lim _{y \rightarrow x} F_{i k}(y) \neq \lim _{y \rightarrow x} F_{i l}(y)$.

Theorem 15. If all singularities $S(F) \cap \mathcal{Y}$ are unstable, then $h_{\mathrm{top}}(F)=H_{\mathrm{sing}}(F)$.

We need the following technical lemma:

Lemma 16. If the singularities in $\mathcal{Y}$ are unstable, then there exists a constant $\gamma>0$ such that for all $\delta>0, x \in \mathcal{Y} \cap M_{i k}$ and $y \in \mathcal{Y} \cap M_{i l}, k \neq l$, we have:

$$
d(x, y)<\delta \Rightarrow d\left(F_{i}(x), F_{i}(y)\right)>\delta .
$$

Proof. Suppose that for all $\delta>0$ there exists $x \in M_{i k} \cap \mathcal{Y}$ and $y \in M_{i l} \cap \mathcal{Y}$ such that $d(x, y)<\delta$ and $d\left(F_{i k}(x), F_{i l}(y)\right) \leq \delta$. There exist sequences $\left\{x_{m}\right\} \subset$ $M_{i k}$ and $\left\{y_{m}\right\} \subset M_{i l}$ such that $d\left(x_{m}, y_{m}\right) \rightarrow 0$ and $d\left(F_{i k}\left(x_{m}\right), F_{i l}\left(y_{m}\right)\right) \rightarrow 0$. The sequence $\left\{x_{m}\right\}$ has a convergent subsequence $x_{m_{n}} \rightarrow z$. The point $z$ lies in $S(F)$ and $y_{m_{n}} \rightarrow z$. By the continuity of the maps $F_{i k}$ and $F_{i l}$ we have $F_{i k}\left(x_{m_{n}}\right) \rightarrow F_{i k}(z)$ and $F_{i l}\left(y_{m_{n}}\right) \rightarrow F_{i l}(z)$. Since the metric $d$ is continuous on $M \times M$ we have

$$
d\left(F_{i k}(z), F_{i l}(z)\right)=\lim _{n \rightarrow \infty} d\left(F_{i k}\left(x_{m_{n}}\right), F_{i l}\left(y_{m_{n}}\right)\right)=0 .
$$




\section{Boris Kruglikov, Martin Rypdal}

Hence $F_{i k}(z)=F_{i l}(z)$. Contradiction.

\section{Proof of Theorem 15. Let}

$$
\left[P_{a_{0}} \ldots P_{a_{n-1}}\right]=\left[i_{0} \ldots i_{n-1}\right] \times K
$$

where $K \subset M$ is a convex polygon. Fix $\delta>0$ and set $k=[\log 1 / \delta]$. Let $z_{1}, \ldots, z_{m(\delta)}$ be a $\delta$-spanning set for $K$. Chose $\mathbf{t} \in \Sigma_{N}^{+}$and define $N^{k}$ sequences

$$
\mathbf{s}_{r_{0} \ldots r_{k-1}}=\left(i_{0} \ldots i_{n-1} r_{0} \ldots r_{k-1} t_{n+k} t_{n+k-1} \ldots\right) \in \Sigma_{N}^{+} .
$$

Since the maps $F_{i j}$ are contracting it is clear that the set $\left\{\hat{x}=\left(\mathbf{s}_{r_{0} \ldots r_{k-1}}, z_{l}\right)\right\}$ is a $(n, \delta)$-spanning set for $\left[P_{a_{0}} \ldots P_{a_{n-1}}\right]$, and since the minimum number of balls needed to cover a convex polygon $K \subset M$ is bounded by $m(\delta) \leq C_{N} \delta^{-N}$ we see that the number of $(n, \delta)$-balls to cover $\left[P_{a_{0}} \ldots P_{a_{n-1}}\right]$ is bounded by $m(\delta) N^{k} \cdot \#\left\{\left[P_{a_{0}} \ldots P_{a_{n-1}}\right]\right\}$. Therefore we get an estimate for the number of $(n, \delta)$-balls to cover $\hat{M}$ and so

$$
h_{\text {top }}(F) \leq \lim _{n \rightarrow \infty} \frac{\log \left(C_{\delta} \delta^{-N} \operatorname{card}\left(\mathcal{P}^{n}\right)\right)}{n}=H_{\text {sing }}(F) .
$$

To see the opposite inequality let $A, B \in \mathcal{P}^{n}$ and take $\hat{x}_{1}=(\mathbf{t}, x) \in A$ and $\hat{x}_{2}=(\mathbf{s}, y) \in B$. Suppose that $A \neq B$ and that $t_{0}=s_{0}, \ldots, t_{n-1}=s_{n-1}$. Then there is $m<n$ such that $\pi_{s} \circ F^{m}(\hat{x}) \in M_{t_{m} k}$ and $\pi_{s} \circ F^{m}(\hat{y}) \in M_{t_{m} l}, k \neq l$. By Lemma 16 there is $\gamma>0$ such that for all $\xi<\gamma$ :

$$
\max \left\{d\left(\pi_{s} \circ F^{m}(\hat{x}), \pi_{s} \circ F^{m}(\hat{y})\right), d\left(\pi_{s} \circ F^{m+1}(\hat{x}), \pi_{s} \circ F^{m+1}(\hat{y})\right)\right\} \geq \xi .
$$

Therefore for $\delta$ sufficiently small, no $(n+1, \delta)$-ball can contain points of both $A$ and $B$ and the minimal $(n+1, \delta)$-spanning set has at least $\operatorname{card}\left(\mathcal{P}^{n}\right)$ elements. Then $h_{\text {top }}(F) \geq H_{\text {sing }}(F)$.

Theorem 17. For the Zhang models: $h_{\text {top }}(\hat{f})=H_{\text {sing }}(\hat{f}), h_{\text {top }}(F)=H_{\text {sing }}(F)$.

Proof. By Remark7 7 all quantities are topological entropies. The corresponding systems in the second equality are the Poincaré return maps for the transformations of the first equality. The map $F: \hat{M} \rightarrow \hat{M}$ is already the return map for $\hat{f}: \hat{B} \rightarrow \hat{B}$ by the very construction.

To achieve the same claim for the symbolic system we extend the partition $\mathcal{P}$ of $\hat{M}$ to a partition $\tilde{\mathcal{P}}$ of $\hat{B}$, which on $\Sigma_{N}^{+} \times\left(\mathbb{R}_{>0}^{N} \backslash M\right) \cap \hat{B}$ equals the product of the standard partition $\Sigma_{N}^{+}=\cup_{i}[i]$ and the partition of the spacial part by the hyperplanes $\left\{x_{i}=E_{c}\right\}$. Denote by $s \leq r+N\left(2^{N}-1\right)$ the number of elements of the new partition $\mathcal{P}$.

Let $\mathcal{B} \subset \hat{B}$ be the $\hat{f}$-invariant closure of $\mathcal{A}$ in $\Sigma_{N}^{+} \times \mathbb{R}_{\geq 0}^{N}$. Define a map $\hat{g}$ : $\mathcal{B} \rightarrow \Sigma_{s}^{+}$by letting $\hat{g}(\hat{x})$ be the unique sequence $b_{0} b_{1} \ldots$ such that $\hat{f}^{n}(\hat{x}) \in \tilde{\mathcal{P}}_{b_{n}}$. We wish to prove that

$$
h_{\text {top }}(\hat{f})=H_{\text {sing }}(\hat{f})=h_{\text {top }}\left(\sigma_{s}^{+} \mid \hat{g}(\mathcal{B})\right) .
$$


For this it is sufficient to check that the singularities $S(\hat{f}) \cap \pi_{s}(\mathcal{B})$ are unstable for $\hat{f}$ (the second equality follows from the definition).

Consider a singular point $x \in \mathbb{R}_{\geq 0}^{N}$. Let $y, z$ tend to $x$ by two different domains of the projected partition $\tilde{\mathcal{P}}$ in $\mathbb{R}_{\geq 0}^{N}$. Then we can subdivide $\{1, \ldots, N\}=A \cup B \cup C$, where $y_{i}=E_{c}+0, \bar{z}_{i}=E_{c}-0$ (with the obvious notations instead of limits) for $i \in A, y_{i}=E_{c}-0, z_{i}=E_{c}+0$ for $i \in B$ and $y_{i}, z_{i}$ belong to the same side of $E_{c}$ for $i \in C$. Then for $\tilde{S}=S(y)-S(z)$ we have:

$$
\tilde{S}_{i j}= \begin{cases}\epsilon-1, & \text { if } i=j \in A, \\ 1-\epsilon, & \text { if } i=j \in B, \\ \frac{1-\epsilon}{2 d}, & \text { if } d_{\Lambda}(i, j)=1, j \in A, \\ \frac{\epsilon-1}{2 d}, & \text { if } d_{\Lambda}(i, j)=1, j \in B, \\ 0 & \text { otherwise. }\end{cases}
$$

We should check that $\tilde{S} \cdot v \neq 0$ for any vector $v \in \mathbb{R}_{>0}^{N}$ with components $v_{i}=E_{c}$ for $i \in A \cup B$ (this implies that $x$ is unstable). Note that other components $v_{i}$, $i \in C$ do not contribute to the product.

Let $i$ be a site from $A$ with not all neighbors from $A$ (if the set $A$ is empty consider $B$ ). Denote the number of $A$-neighbors of $i$ by $k_{A}<2 d$ and the number of $B$-neighbors by $k_{B} \geq 0$. Then $(\tilde{S} \cdot v)_{i}=E_{c}\left(2 d-k_{A}+k_{B}\right) \frac{\epsilon-1}{2 d} \neq 0$.

Thus Theorem [15] implies [6). Moreover, the same reasons yield a more general statement. Namely, since $\hat{g}$ is a semi-conjugancy, we get:

$$
h_{\text {top }}(\hat{f} \mid \mathcal{K})=h_{\text {top }}\left(\sigma_{s}^{+} \mid \hat{g}(\mathcal{K})\right)
$$

for any subset $\mathcal{K} \subset \mathcal{B}$ (recall that $\hat{g}$ may have discontinuities, but our arguments are not injured by this fact). This subset needs not to be invariant, and in this case we should use Bowen's definition of entropy [Bo]. Thus Katok's entropy formula [K] implies that $h_{\hat{\mu}}(\hat{f})=h_{\hat{g}_{*} \hat{\mu}}\left(\sigma_{s}^{+}\right)$for all $\hat{f}$-invariant measures $\hat{\mu}$, which are not supported on singularities.

If the system $(\hat{M}, F)$ possesses a measure $\mu$ of maximal entropy, then by the obtained result the second claim of the theorem follows from (5) and Kac's theorem [PY]. In general, we can apply the above arguments to the partition of $\hat{M}$ by the subsets of equal return times and using the fact, that both returns of $\hat{f}$ and $\hat{g}$ have the same combinatorics, we get: $h_{\text {top }}(F)=h_{\text {top }}\left(\sigma_{r}^{+} \mid g(\mathcal{A})\right)$ (see, for instance, the loop equation approach $[\mathrm{Pt}]$ ).

\subsection{Evaluation of topological entropy}

It was predicted on the base of variational principle in $[\mathrm{BCK}$ that topological entropy of the Zhang model is $h_{\text {top }}(F)=\log N$. However this principle does not apply because the map is not well-defined (continuously) on the whole space (or thanks to non-compactness if we remove the singularities). In fact, there can be no invariant measures on the non-singular part at all.

While we support the claim that $h_{\mathrm{top}}(F)=\log N$, it will not be proved in full generality. We start with the asymptotic statement. 


\section{Boris Kruglikov, Martin Rypdal}

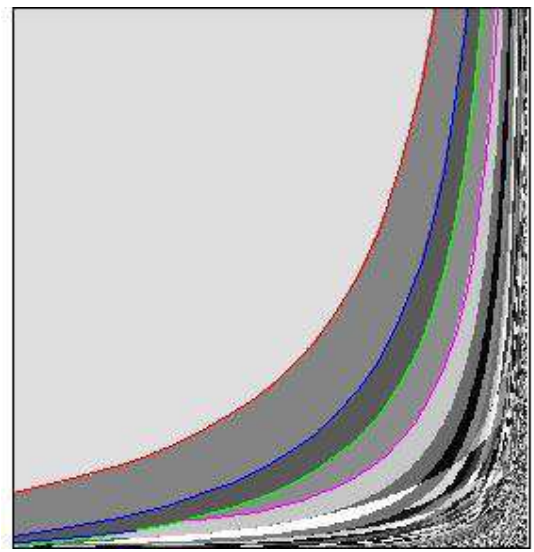

Figure 1: Shows the avalanche type domains on $\left(\epsilon, E_{c}\right)$-bifurcation diagram for $N=2$. The top domain is $E_{c}>\frac{1+\epsilon}{1-\epsilon}$. The line from infinity to the origin is $E_{c}=\frac{\epsilon}{1-\epsilon}$. We see infinitely many domains with different avalanches, accumulating to two of the axes.

Consider the bifurcation diagram on the $(E, \epsilon)$ strip $(0,+\infty) \times[0,1)$, where a point is critical if in its neighborhood dynamics of the Zhang model can experience avalanches of different types. Thus the strip is partitioned into different avalanche type domains. The partition depends on $L, d$. For $N=2$ the diagram is shown on Figure 1

Note that for all $L, d$ there is the top avalanche type domain representing the shortest avalanche time. For $N=2$ it is given by the relation $E>\frac{1+\epsilon}{1-\epsilon}$. Also note that some domains have $\epsilon$-projection strictly smaller than the interval $[0,1)$. The next statement concerns only the top domain and the avalanche type domains that are adjacent to the line $\epsilon=1$.

Theorem 18. For the Zhang model: $0 \leq h_{\mathrm{top}}(F)-\log N \leq \theta(E, \epsilon)$, where $\lim _{E_{c} \rightarrow \infty} \theta\left(E_{c}, \epsilon\right)=0$ ( $\epsilon$ fixed) and $\lim _{\epsilon \rightarrow 1} \theta\left(E_{c}, \epsilon\right)=0$. In the latter case $E_{c}$ changes accordingly with $\epsilon$ so that $\left(E_{c}, \epsilon\right)$ belongs to the same avalanche type domain (then $E_{c} \rightarrow \infty$, though this case differs from the former).

We can remove the $N$-rational points from $\Sigma_{N}^{+}$and then represent it as the subset of $I=[0,1]$ with points $n / N^{k}$ being deleted (this process does not change the topological entropy). In fact, we need to remove only points $n / N$ for the others will be deleted by inverse iterations of the map of $I \times X$ with the formula $F(t, x)=\left(N t, f_{[N t]}(x)\right)$. Thus we represent our system as a piece-wise affine partially hyperbolic map of the subset of $\mathbb{R}^{1+N}$. This is important for an application of the results from Appendix $\mathrm{A}$

The proof uses the notions of multiplicity and multiplicity entropy, due to G. Keller and J. Buzzi. Given a finite partition $\mathcal{Z}=\left\{Z_{k}\right\}$ of $\hat{M}$ we define

$$
\mathcal{Z}^{n}=\left\{\left[Z_{0} \ldots Z_{n-1}\right] \neq \emptyset\right\},
$$


where

$$
\left[Z_{0} \ldots Z_{n-1}\right]=Z_{0} \cap F^{-1}\left(Z_{1}\right) \cap \cdots \cap F^{1-n}\left(Z_{n-1}\right) .
$$

Define multiplicity of a partition by

$$
\operatorname{mult}(\mathcal{Z})=\max _{\hat{x} \in \hat{M}} \operatorname{card}\{Z \in \mathcal{Z} \mid \bar{Z} \ni x\},
$$

If $\mathcal{Z}$ is the continuity partition of the map $F$ we often denote the multiplicity of the $\mathcal{Z}$ by $\operatorname{mult}(F)$. Then it is clear that $\operatorname{mult}\left(F^{n}\right)=\operatorname{mult}\left(\mathcal{Z}^{n}\right)$. The multiplicity entropy of $F$ is (the limit exists by subadditivity, cf. $[\mathrm{KH}]$ )

$$
H_{\text {mult }}(F)=\sup _{\mathcal{Z}} \lim _{n \rightarrow \infty} \frac{1}{n} \log \operatorname{mult}\left(\mathcal{Z}^{n}\right),
$$

and we see that if $\mathcal{Z}$ is the continuity partition, then

$$
H_{\text {mult }}(F)=\lim _{n \rightarrow \infty} \frac{1}{n} \log \operatorname{mult}\left(F^{n}\right) .
$$

It is clear that $H_{\text {mult }}(F)=0$ if the singularities are removable, so $h_{\text {top }}(F)=$ $\log N$ by the result in Appendix $\mathrm{A}$ For big $E_{c} \gg 1$ the singularities are generally non-removable, but still we have the same effect asymptotically:

Proof of Theorem 18, We take $\theta\left(E_{c}, \epsilon\right)=H_{\text {mult }}(F)$. Since the singularities $t=n / N \in I$ of the map $\sigma_{N}^{+}: t \mapsto N t$ do not intersect in inverse iterations, the multiplicity growth is only due to the spacial maps $F_{i}: M \rightarrow M$. Thus using the notation $F_{\mathbf{t}}$ from Section 3.2 for the dynamics over a prefixed sequence of excitations we obtain $H_{\text {mult }}(F)=\sup _{\mathbf{t} \in \Sigma_{N}^{+}} H_{\text {mult }}\left(F_{\mathbf{t}}\right)$.

To show the first claim let us notice that when $\epsilon=$ const, but $E_{c} \rightarrow \infty$, then the avalanches map the critical part of the boundary $\left\{\exists i: x_{i}=E_{c}\right\} \subset \partial M$ far from $\partial M$, namely to the distance $\sim \gamma(\epsilon, L, d) E_{c}$, see Figures 16 for $N=2$ and 17 for $N=3$. To reach again the boundary and experience avalanche we need many shifts $F_{i}$ by the basic vectors $e_{i}$. Thus the singularity can meet only after big number of iterations. Since the initial picture of singularities has bounded multiplicity (for $\left(\epsilon, E_{c}\right)$ from the top avalanche type domain), the multiplicity decreases at least as $k / E_{c}$ so that it vanishes in the limit.

To prove the second claim we use the inequality $H_{\text {sing }}(F) \leq \sum \rho_{i}(F)$ from Appendix A.3. If the avalanche type domain is fixed, the number of compositions of matrices $S(x)$ in one avalanche (see 1.3 is bounded. Every such a matrix tends to identity when $\epsilon \rightarrow 1$. Thus all the linear parts $L_{i j}$ of avalanche maps $F_{i j}$ tend to identity and so angular expansions $\rho_{i}(F)$ tend to zero.

Notice that if $E_{c}$ is fixed, but $\epsilon \rightarrow 1$, then the number of avalanches has unlimited grow and the previous argument do not work. However, due to estimates of 1.2 on the maximal avalanche length $\tau_{m}$ we conclude that $\theta\left(E_{c}, \epsilon\right) \rightarrow 0$ as either $E_{c} \rightarrow \infty$ or $\epsilon \rightarrow 1$, both quantities being related by the constraint $E_{c} \geq N(1-\epsilon)^{-\frac{1}{2} \operatorname{diam}(\Lambda)-\sigma}$ for some $\sigma>0$ (we don't require, but allow $N \rightarrow \infty$ as well). This statement is stronger than in the theorem.

Now we are going to prove vanishing of $\theta\left(E_{c}, \epsilon\right)$ a.e. in the finite part. 


\section{Boris Kruglikov, Martin Rypdal}

Theorem 19. For generic $\left(E_{c}, \epsilon\right)$ we have: $h_{\mathrm{top}}(F)=\log N$.

We will prove the statement not only for the Zhang model, but also for nearly Zhang models. By this we mean the following. The map $F$ is a bundle over the Bernoulli shifts $\sigma_{N}^{+}$with factors $F_{i}$ being piece-wise affine partially contracting maps. That is $M=\cup_{j} M_{i j}$ and for $F_{i j}=\left.F_{i}\right|_{M_{i j}}$ we have $F_{i j}(x)=L_{i j}(x)+b_{i j}$, $b_{i j}=L_{i j} e_{i}$. We are going to make arbitrary small generic perturbations of the matrices $L_{i j}$ (still with spectrum within unit ball) and vectors $b_{i j}$ (one should care that $M_{i j}$ are mapped into $M$ ) and prove the statement for this modified system.

Due to round-off errors there is no much difference between the original and the perturbed systems in computer simulations. And from the point of view of the experiment such perturbations (instrument instability) are indispensable look at $[\mathrm{Ru}]$ for the discussion of physical relevance of variation of parameters as the noise.

Proof of Theorem 19] We claim that for generic $\left(E_{c}, \epsilon\right)$ the singularities do not multiply. Actually, some intersections of singularities are deformable as we vary the parameters, just by the transversality reasons, but the other disappear with small perturbations.

Namely, for a multi-index $\sigma=\left(\alpha_{1}, \ldots, \alpha_{k}\right), \alpha_{s}=\left(i_{s}, j_{s}\right)$ coding an orbit, denote $F_{\sigma}=F_{\alpha_{k}} \circ \cdots \circ F_{\alpha_{1}}$ the corresponding map along the orbit. The singularities of this map are: $\operatorname{Sing}\left(F_{\sigma}\right)=\cup_{r=1}^{k} F_{\sigma_{[r]}}^{-1} \operatorname{Sing}\left(F_{\alpha_{r}}\right)$, where $\sigma_{[r]}=\left(\alpha_{1}, \ldots, \alpha_{r}\right)$.

If $z_{0} \in \operatorname{Sing}\left(F_{\sigma}\right)$, then its orbit (with multi-possibilities due to singularities: mapping a singular point we extend the components of the map $F_{\sigma}$ in various ways) meets several singularity planes, i.e. for some cuts $\sigma_{s}=\sigma_{\left[r_{s}\right]}$ of $\sigma, s=$ $1, \ldots, m$, we have the following system:

$$
F_{\sigma_{1}}\left(z_{0}\right)=z_{1}, \ldots, F_{\sigma_{m}}\left(z_{0}\right)=z_{1}, l_{q_{1}}\left(z_{1}\right)=0, \ldots, l_{q_{m}}\left(z_{m}\right)=0
$$

where $l_{q_{s}}(z)$ are equations for the singularity hyperplanes of the corresponding map $F_{i}$ (there are also inequalities, which we don't mention). When $m \leq N$ there are occasions, when (8) has a solution continuously depending on $\left(E_{c}, \epsilon\right)$. However for $m>N$ this is no longer the case. In fact, considering nearly-Zhang models we see that for generic data the above system (8) is characterized by a collection of non-trivial polynomial in $\epsilon$ equations (for each $E_{c}$ ).

More precisely, the set of $\left(L_{i j}, b_{i j}\right)$ giving trivial polynomials has positive codimension and hence zero Lebesgue measure. Uniting these sets over all choices of multi-indices $\left(\sigma_{1}, \ldots, \sigma_{m}\right)$ we see that the complement has full measure and dimension and so a generic perturbation yields the data $\left(L_{i j}, b_{i j}\right)$ from it. Since non-trivial polynomials have only finite number of zeros, then for a generic nearly Zhang model and every $E_{c}$ there is a countable subset of $\{0 \leq \epsilon<1\}$, so that the corresponding systems (8) have no solutions. This means that multiplicity of $F_{\mathbf{t}}^{n}$ does not grow with $n$ and so $H_{\text {mult }}\left(F_{\mathbf{t}}\right)=0$.

Now let us look to the Zhang model. We restrict for simplicity of exposition to the case of two sites $N=2$. In this case the linear parts of the affine maps 
are compositions of $L_{1}=\mathbf{1}+(\epsilon-1) A_{1}$ and $L_{2}=\mathbf{1}+(\epsilon-1) A_{2}$ with

$$
A_{1}=\left[\begin{array}{cc}
1 & 0 \\
-1 / 2 & 0
\end{array}\right], \quad A_{2}=J^{-1} A_{1} J=\left[\begin{array}{cc}
0 & -1 / 2 \\
0 & 1
\end{array}\right], \text { where } J=\left[\begin{array}{cc}
0 & 1 \\
-1 & 0
\end{array}\right]
$$

(we exclude the obvious matrix 1, see Example A for details). Notice that $\operatorname{det} L_{1}=\operatorname{det} L_{2}=\epsilon$.

Suppose that (8) has a continuous solution in some domain of $\left(E_{c}, \epsilon\right)$. Then it is algebraic in $\epsilon$ and linear in $E_{c}$ (the latter is because the singularity lines within one avalanche type domain shift with velocity 1 in the direction of either $e_{1}=\left(\begin{array}{l}1 \\ 0\end{array}\right)$ or $e_{2}=\left(\begin{array}{l}0 \\ 1\end{array}\right)$. Differentiating this by $E_{c}$ we obtain:

$$
L_{\rho_{1}}\left(z_{0}^{\prime}\right)=z_{1}^{\prime}, \ldots, L_{\rho_{m}}\left(z_{0}^{\prime}\right)=z_{m}^{\prime},
$$

where $L_{\rho_{i}}=L_{\rho_{i, t_{i}}} \circ \cdots \circ L_{\rho_{i, 1}}$ for $\rho_{i}=\left(\rho_{i, 1}, \ldots, \rho_{i, t_{i}}\right)$ is the linear part of $F_{\sigma_{i}}$ ( $\rho_{i}$ is different from $\sigma_{i}$ because $d F_{\sigma_{i}}$ is a composition of several maps $L_{s}$ ). The points $z_{k}, 1 \leq k \leq m$, are constrained to the singularity lines and we can suppose these are the lines $\left\{x_{1}=E_{c}\right\}$ or $\left\{x_{2}=E_{c}\right\}$ (all other singularities are mapped to them within one avalanche). Thus $z_{k}^{\prime}=v_{k}+\psi_{k} w_{k}$, where $v_{k}=e_{1}$ or $e_{2}$ and $w_{i}=J v_{i}$ is the other basic vector, $\psi_{k}$ being an unknown scalar.

Let $\xi_{k}=L_{\rho_{k}}^{-1}\left(v_{k}\right), \zeta_{k}=L_{\rho_{k}}^{-1}\left(w_{k}\right)$; these vectors depend meromorphically on $\epsilon$. System (9) is solvable iff the affine lines $\xi_{k}+\psi_{k} \zeta_{k}, 1 \leq k \leq m$, in $\mathbb{R}^{2}$ have a common point. We can suppose $m=3$.

Denote by $\Omega(\xi, \eta)=\langle J \xi, \eta\rangle$ the standard symplectic form on $\mathbb{R}^{2}$. The above 3 lines intersect jointly iff

$$
\Omega\left(\xi_{1}, \zeta_{1}\right) \Omega\left(\zeta_{2}, \zeta_{3}\right)+\Omega\left(\xi_{2}, \zeta_{2}\right) \Omega\left(\zeta_{3}, \zeta_{1}\right)+\Omega\left(\xi_{3}, \zeta_{3}\right) \Omega\left(\zeta_{1}, \zeta_{2}\right)=0 .
$$

Dividing by $\prod_{k=1}^{3} \Omega\left(\xi_{k}, \zeta_{k}\right)$ and using the fact that $\Omega\left(\xi_{k}, \zeta_{k}\right)=\operatorname{det}\left(L_{\rho_{k}}^{-1}\right)=$ $\epsilon^{-\left|\rho_{k}\right|}$ we get the equivalent equation:

$$
\Omega\left(\eta_{1}, \eta_{2}\right)+\Omega\left(\eta_{2}, \eta_{3}\right)+\Omega\left(\eta_{3}, \eta_{1}\right)=0
$$

Here $\eta_{k}=\epsilon^{-\left|\rho_{k}\right|} L_{\rho_{k}}^{-1} w_{k}=\tilde{L}_{\rho_{k}} w_{k}$, where $\tilde{L}_{\tau}=\tilde{L}_{\tau_{1}} \circ \cdots \circ \tilde{L}_{\tau_{t}}$ for $\tau=\left(\tau_{1}, \ldots, \tau_{t}\right)$ and $\tilde{L}_{k}=\epsilon^{-1} L_{k}^{-1}=\mathbf{1}+(\epsilon-1) \tilde{A}_{k}$ is the adjunct matrix for $L_{k}$, which gives

$$
\tilde{A}_{1}=\left[\begin{array}{cc}
0 & 0 \\
1 / 2 & 1
\end{array}\right], \quad \tilde{A}_{2}=\left[\begin{array}{cc}
1 & 1 / 2 \\
0 & 0
\end{array}\right] .
$$

Now equation (10) holds iff there exist 3 not simultaneously zero numbers $\beta_{1}, \beta_{2}, \beta_{3}$ such that $\beta_{1}+\beta_{2}+\beta_{3}=0$ and $\beta_{1} \eta_{1}+\beta_{2} \eta_{2}+\beta_{3} \eta_{3}=0$. Since $\eta_{k}$ is a polynomial matrix of degree $\left|\rho_{k}\right|$ and the products of $A_{t}$ are always proportional to $e_{1}$ or $e_{2}$ (depending on the left-most factor) this last equation is never satisfied if the multi-indices $\rho_{k}$ are different.

Remark 8. To support the usage of nearly-Zhang models note that the whole paradigm of SOC should allow generic perturbations of the data, for if there is a fine tuning of parameters, the model is unappropriate for physical explanation (of course, we should pass to the thermodynamic limit, but in practice this only means some large finite parameters). 


\section{Boris Kruglikov, Martin Rypdal}

Our computer experiments did not expose any exponential growth of multiplicity in the Zhang model (though we see growth in complications of singularities), so we suggest that $H_{\text {mult }}(F)=0$ and hence $h_{\text {top }}(F)=\log N$ always. In addition, by the above discussion we can disregard these exceptional values of $\left(E_{c}, \epsilon\right)$ even if there are any. This finishes discussion of topological entropy.

\section{Geometry of the attractor}

The construction of the spacial attractor $\mathcal{Y}$ can be interpreted as an iterated function system (IFS), where the maps $F_{i}$ are not affine as usually considered, but piecewise affine. Hence one might expect that attractors $\mathcal{Y}$ are fractal, but with various size characteristics, like dimension and measure, depending on parameters $E_{c}$ and $\epsilon$.

\subsection{Fractal structure}

Computer experiments show that in certain cases the spacial attractor $\mathcal{Y}$ has fractal structure, see e.g. Figures 2 and 3 . We clearly see that $\mathcal{Y}$ consists of self-similar pieces. However the pieces overlap, making evaluation of the fractal dimension difficult. So we can provide only estimates of the attractor's size.

Nevertheless we observe from our experiments that Hausdorff dimension $\operatorname{dim}_{\mathrm{H}}(\mathcal{Y})$ and the Lebesgue measure $\mu_{\mathrm{Leb}}(\mathcal{Y})$ of the attractor grow piece-wise monotonically with $E_{c}$ and $\epsilon$. Thus the following effects occur in steps:

- The dimension and the measure of $\mathcal{Y}$ vanish.

- $\operatorname{dim}_{\mathrm{H}}(\mathcal{Y})$ is positive, while the measure is zero.

- Both $\operatorname{dim}_{\mathrm{H}}(\mathcal{Y})$ and $\mu_{\mathrm{Leb}}(\mathcal{Y})$ are positive.

- The attractor $\mathcal{Y}$ contains an interior point.

We will demonstrate the dimensional part in the next section, while we disregard the observation about the measure. The reason for this is that $\mu_{\text {Leb }}$ is not physically motivated and we should look for an SRB-measure.

The experiments show that such a measure exists and has support lying strictly inside $\mathcal{Y}$. (See Figure 4 and Figure 5 In Figure 4 the attractor $\mathcal{Y}$ is shown for the case $N=2, E_{c}=20$ and $\epsilon=2 / 3$, and Figure 5 shows the orbit of a random initial condition. The latter corresponds to the support of the SRB-measure.) This is possible because the contraction rate of $f_{\mathbf{t}}$ is smaller for the exceptional sequences $\mathbf{t} \in \Sigma_{N}^{+}$, than for a generic one. Thus study of the IFS-attractor does not lead to conclusions about ergodicity or uniqueness of the SRB-measure. Still it provides an information about spacial distribution of the orbits in the Zhang dynamics. 
Dynamics and entropy of SOC

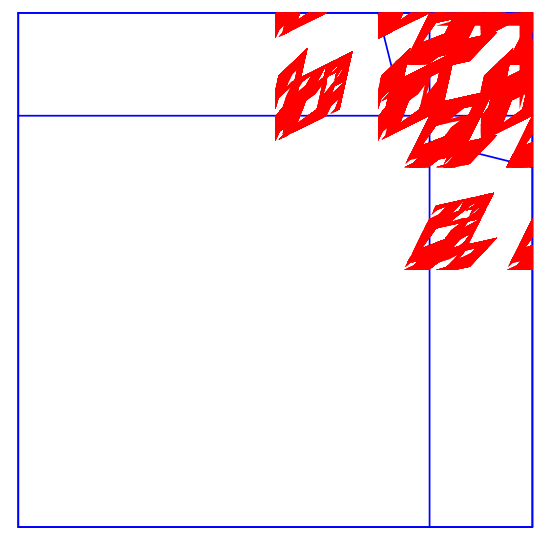

Figure 2: Shows the set $U_{10}$ for $N=2, E_{c}=5$ and $\epsilon=1 / 2$.

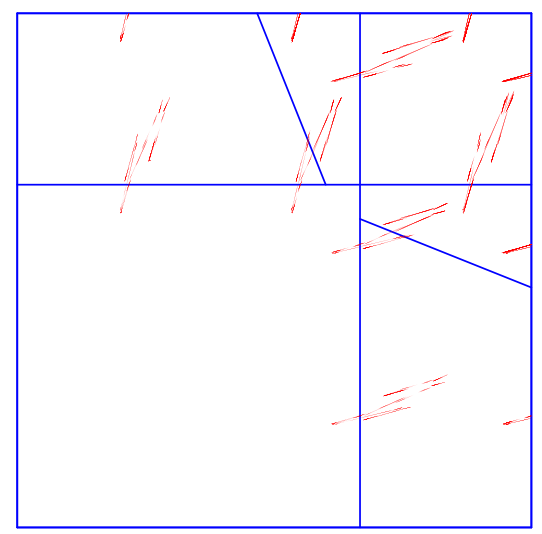

Figure 3: Shows the set $U_{10}$ for $N=2, E_{c}=3$ and $\epsilon=1 / 5$. 
Boris Kruglikov, Martin Rypdal

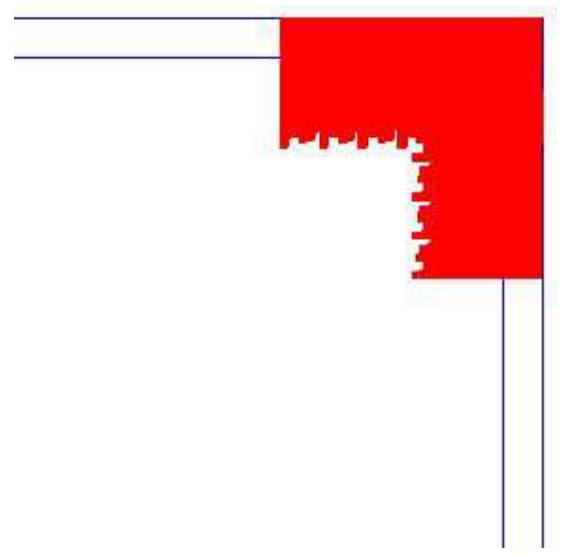

Figure 4: Shows the set $\mathcal{Y}$ in the top right corner of $M$ for $N=2, E_{c}=20$ and $\epsilon=2 / 3$. The dimension of $\mathcal{Y}$ is 2 in this example.

\subsection{Dimensional study of the attractor}

The fractal properties of $\mathcal{Y}$ do not hold for all values of parameters $\left(E_{c}, \epsilon\right)$. An example where $\mathcal{Y}$ has integer dimension is shown in Figure 4

It was noted in $\mathrm{BCK}$ that Hausdorff (fractal) dimension of the attractor is about to increase as $E_{c}$ grows. The arguments were the following: For bigger $E_{c}$ the contraction rate decreases, so the theory of iterated function system (IFS) implies increasing of the Hausdorff dimension

$$
\mathfrak{D}_{\mathcal{Y}}\left(\epsilon, E_{c}\right)=\operatorname{dim}_{\mathrm{H}}(\mathcal{Y})
$$

as a function of $E_{c}$. While this seems to be true, the statement does not hold in precise sense. For instance, for $N=2, E_{c} \in\left[\frac{1+\epsilon}{1-\epsilon}, \frac{2}{1-\epsilon}\right]$ the attractor is the set of 3 points, while it seems to have non-zero dimension for other parameters (computer simulations clearly show this).

The problem is with the framework of IFS, where usually only conformal maps are considered and certain regularity of their mapping graph and overlaps is assumed. However we will show validity of the claim in the asymptotic sense:

Theorem 20. With fixed $d, L$ and generic $\epsilon$ we have: $\lim _{E_{c} \rightarrow \infty} \mathfrak{D}_{\mathcal{Y}}\left(\epsilon, E_{c}\right)=N$. Moreover, $\mathfrak{D}_{\mathcal{Y}}\left(\epsilon, E_{c}\right)=N$ for big values $E_{c} \gg 1 / \epsilon$.

On the other hand for all $\epsilon \in[0,1)$ it holds: $\lim _{E_{c} \rightarrow 0} \mathfrak{D}_{\mathcal{Y}}\left(\epsilon, E_{c}\right)=0$.

In the above statement "generic" means both full Lebesgue measure and second Baire category. In fact, the equality holds for all $\epsilon$ outside a countable set. It seems though that the limit statement is valid for all $\epsilon$. Thus we see that $\mathfrak{D}_{\mathcal{Y}}\left(\epsilon, E_{c}\right)$ is not strictly monotone in $E_{c}$ for its large values as one might expect 
Dynamics and entropy of SOC

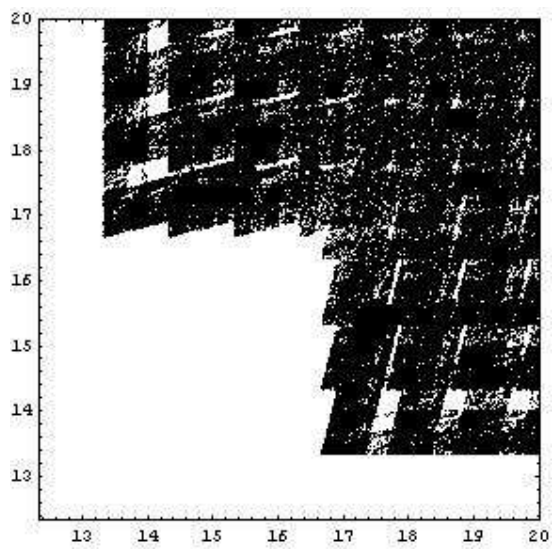

Figure 5: Shows an orbit of a randomly chosen point (i.e. the support of the SRBmeasure) for $N=2, E_{c}=13$ and $\epsilon=2 / 3$.

from the arguments cited before the theorem.

Proof. Consider first the statement about big energies $E_{c} \gg 1 / \epsilon$. Let us start by demonstrating the idea of the proof on the example $N=2$, see Figure 16

The image of the vertical continuity domain $M_{13}$ of height 1 adjusted to the right-top corner is a trapezium with the slope depending on $\epsilon$ (see (11) of Section 6 for the numeration of domains). It is thin - of constant length of horizontal section equal $\epsilon$ near its bottom side, but long - with diameter approximately equal $E_{c} \frac{1-\epsilon^{2}}{4}$. Thus if we shift $\sim C / \epsilon$ times this domain up and then all of the shifted images horizontally to the right, so that its first coordinate satisfies the inequality $E_{c}-1<x_{1} \leq E_{c}$, then these shifts cover an open domain, including a unit square, in the vertical strip $K_{1}=\left\{x_{1} \in\left(E_{c}-1, E_{c}\right]\right\}$ (note that we can leave a copy of the domain since this corresponds to the shift - dropping of energy $\left(x_{1}, x_{2}\right) \mapsto\left(x_{1}, x_{2}+1\right)$ on $M_{13} \subset K_{1}$ before the avalanche). An easy calculation shows that such shifts cover the whole upper part of $K_{1}$, strictly including the continuity domain $M_{13} \subset K_{1}$ adjusted to $\left(E_{c}, E_{c}\right)$ and covering a vertical part of $M_{12}$. A similar scenario happen to the second coordinate. Thus in iterating the dynamics we will always have two continuity domains $M_{13}$ and $M_{23}$ adjusted to $\left(E_{c}, E_{c}\right)$ and the adjacent parts of $M_{12}, M_{22}$ lying in the attractor.

For general $L$ and $d$ we observe the same picture: With generic $\epsilon$ a continuity domain $M_{i j}$ adjusted to the upper-most corner is mapped under avalanche-map $F_{i j}$ to the trapezoid-like polyhedron with irrational slopes. Its shifts cover then an open domain in each of the strips $K_{i}=\left\{x_{i} \in\left(E_{c}-1, E_{c}\right]\right\}$ and so after more shifts - the upper part of this strip, whence the statement.

We illustrate this process on Figure 17 The 3 domains adjusted to the corner $\left(E_{c}, E_{c}, E_{c}\right)$ are mapped into interior of the cube and they have different 
Boris Kruglikov, Martin Rypdal

irrational slopes (we picture them of zero thickness that corresponds to large values of $E_{c} \gg 1$ ), so that their shifts cover a big open domain near the faces adjusted to the above corner.

Consider now the second statement, $E_{c} \ll 1$. To estimate the fractal dimension from above we use the generalization of the Moran's formula from Appendix B. It implies that if the IFS $f_{1}, \ldots, f_{N}$ satisfies $\left\|f_{i}\right\| \leq \delta$, then the Hausdorff dimension of the attractor admits the following estimate: $\mathfrak{D}_{\mathcal{Y}} \leq \log N \vartheta / \log \frac{1}{\delta}$, where $\vartheta$ is the maximal multiplicity of the continuity partitions for $f_{i}$.

Now we claim that as $E_{c} \rightarrow 0$ we have: $\delta=\max \left\|f_{i}\right\| \leq\left(E_{c}\right)^{\sigma}$ for some $\sigma>0$. To see this let us estimate the maximal duration of the avalanche $\bar{\tau}_{m}=\max _{(i, x) \in \Lambda \times M} \tau(i, x)$. This quantity tends to $\infty$ as $E_{c} \rightarrow 0$, but not as fast as $\tau_{m}\left(E_{c}, \epsilon, \Lambda\right) \sim C_{1} / E_{c}$ (see 1.2). Namely we state that $\bar{\tau}_{m} \sim C_{2} \log 1 / E_{c}$. Actually, if we drop energy 1 to an arbitrary site from a configuration in $M$, then in a finite $E_{c}$-independent time all the sites become overcritical. They remain overcritical, while the system does not loose a substantial amount of energy. During this process the total energy is dissipating in geometric progression with an average contraction rate $1-\frac{1-\epsilon}{N}<1$. So the duration of this stage has asymptotic $C_{3} \log \left(1 / E_{c}\right)$. The remaining time to finish the avalanche has a smaller asymptotic.

By $1.2 n\left(E_{c}, \epsilon, \Lambda\right)<C_{0}$ for small $E_{c}$. Thus the proof of Theorem 5 implies that for certain $E_{c}$-independent constant $C_{n}=k C_{0}$ for each sequence of $C_{n}$ steps in the avalanche process the product of the corresponding $S$-matrices will have norm $\leq c(\epsilon)<1$, which is a uniform estimate in $E_{c} \ll 1$. The number of steps in one avalanche grows as $\log 1 / E_{c}$. Therefore $\delta \leq C_{4} c(\epsilon)^{C_{n}^{-1} \log 1 / E_{c}} \leq$ $\exp \left(-\sigma \log 1 / E_{c}\right)$, where $\sigma=\frac{1}{2} C_{n}^{-1} \log \frac{1}{c(\epsilon)}$ as was claimed in the estimate.

Next we claim that $\vartheta \leq \varphi\left(E_{c}\right)$ with $\varphi=o\left(\left(1 / E_{c}\right)^{v}\right) \forall v>0$. In fact, we described above the avalanche process for small energies. The first stage is finite and contributes only a bounded number of singularity hyperplanes. In its seconds stage all of the sites are excited, so there the corresponding number of singularity hyperplanes equals the duration. The last stage is shorter of time $\psi=o\left(\log 1 / E_{c}\right)$, but the number of singularity hyperplanes grows faster, but still is bounded by $e^{\psi \log N !}=o\left(\left(1 / E_{c}\right)^{v}\right)$ (see 1.3$)$ for any $v>0$.

Finally $\mathfrak{D}_{\mathcal{Y}}\left(\epsilon, E_{c}\right) \leq \log N \vartheta / \log \frac{1}{\delta} \leq \frac{C^{\prime}+\log \varphi}{\sigma \log 1 / E_{c}} \rightarrow 0$ as $E_{c} \rightarrow 0$.

Corollary 3. For big $E_{c} \gg 1 / \epsilon$ the system $(\mathcal{A}, F)$ is not topologically transitive.

Proof. Suppose $(\mathbf{t}, x)$ is a point with the dense orbit in $\mathcal{A}$. We know that for big $E_{c}$ the Lebesgue measure of the spatial part $\mathcal{Y}$ of the attractor $\mathcal{A}$ is a positive number $\omega>0$. It follows from Theorem 15 that under iterations with fixed excitation sequence $\mathbf{t}$ the volume of the spatial part $M$ decreases in geometric progression with the number of avalanches. Thus after a finite number of steps it becomes less than $\omega$. This iteration will be still a finite number of polyhedra, so that its closure does not coincide with $\mathcal{Y}$. Since it contains all the points $\pi_{s}\left(F^{n}(\mathbf{t}, x)\right)$, we obtain contradiction.

In the case $N=2$ and $\epsilon>1 / 2$, the value of $E_{c}$ starting from which 
Dynamics and entropy of SOC

$\mathfrak{D}_{\mathcal{Y}}\left(\epsilon, E_{c}\right)=2$ can be calculated precisely because even one shift of the sloped strip mentioned in the above proof overlaps with itself and is sufficient for obtaining an open domain in the attractor. This condition $\epsilon>1 / 2$ together with $E_{c} \gg 1$ from the theorem ideologically coincide with the sufficient conditions for invertibility of the differentials of avalanche maps (Proposition 17). This makes an indication of a relation between this invertibility and fractality of the attractor in the spirit of Ledrappier-Young formula [LY]. This latter is however unappropriate in our situation.

Note on the usage of the Ledrappier-Young formula. This formula, essentially used in $\mathrm{BCK}$ in the study of the Zhang model, cannot be used for the map $F: \mathcal{D} \rightarrow \mathcal{D}$ since this map is never invertible (in loc. cit. it was applied to $F^{-1}$ ). In addition to invertibility the Ledrappier-Young theorem is based on the SRB-property. For the map $F^{-1}$ this property is equivalent to absolute continuity of the stable foliation for $F$ w.r.t. the measure $\mu$. If the measure has fractal support this cannot happen. Therefore all formulas based on this property may turn to be wrong. We demonstrate this in Example A of $\$[6$

On the other hand, as we have just shown in the theorem, in thermodynamic limit $E_{c} \rightarrow \infty$ the fractality is lost and so the absolute continuity property is restored (but only for the geometric attractor, the support of SRBmeasure is smaller!). This however does not help with non-invertibility of the factor $\left(\Sigma_{N}^{+}, \sigma_{N}^{+}\right)$. Even if we change this factor to invertible two-sided sequences $\left(\Sigma_{N}, \sigma_{N}\right)$, the system remains non-invertible since not all points of the attractor (which can be quite fat) admit negative iterations (Remark 4). In addition, a new negative Lyapunov exponent $-\log N$ in the first factor appears and the formulas exploited in $\mathrm{BCK}$ become completely inadequate.

\section{Examples}

In the examples below we consider the one-dimensional Zhang model with two sites, $N=2$.

Example A: A computation shows that for $E_{c} \geq(1+\epsilon) /(1-\epsilon)$ we have six domains of continuity $[i] \times M_{i j}, i=1,2$. The domains $M_{1 j}$ are given by

$$
\begin{aligned}
& M_{11}=\left\{x \in\left[0, E_{c}\right]^{2} \mid x_{1}+1 \leq E_{c}\right\} \\
& M_{12}=\left\{x \in\left[0, E_{c}\right]^{2} \mid x_{1}+1>E_{c} \text { and }(1-\epsilon)\left(x_{1}+1\right) / 2+x_{2} \leq E_{c}\right\} \\
& M_{13}=\left\{x \in\left[0, E_{c}\right]^{2} \mid x_{1}+1>E_{c} \text { and }(1-\epsilon)\left(x_{1}+1\right) / 2+x_{2}>E_{c}\right\}
\end{aligned}
$$

and the domains $M_{2 j}$ are symmetric to these. The maps $F_{i j}$ are of the form $F_{i j}(x)=L_{i j}\left(x+e_{i}\right)$, where

$$
L_{11}=\left[\begin{array}{ll}
1 & 0 \\
0 & 1
\end{array}\right] \quad L_{12}=\left[\begin{array}{cc}
\epsilon & 0 \\
\frac{1-\epsilon}{2} & 1
\end{array}\right] \quad L_{13}=\left[\begin{array}{cc}
\left(\frac{1+\epsilon}{2}\right)^{2} & \frac{1-\epsilon}{2} \\
\epsilon \frac{1-\epsilon}{2} & \epsilon
\end{array}\right]
$$

and 
Boris Kruglikov, Martin Rypdal
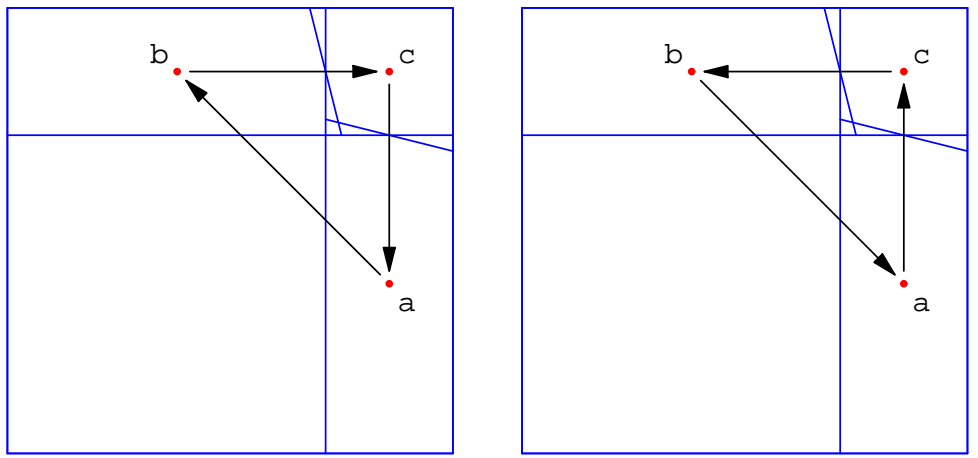

Figure 6: The figure shows the physical attractor $\mathcal{Y}=\{a, b, c\}$ and the maps $F_{1}\left|\mathcal{Y}, F_{2}\right| \mathcal{Y}$ for $E_{c}=7 / 2$ and $\epsilon=1 / 2$. The arrows on the picture to the left shows how the points of $\mathcal{Y}$ are mapped under $F_{1}$, and the picture on the right shows how the points are mapped under $F_{2}$.

$$
L_{11}=\left[\begin{array}{ll}
1 & 0 \\
0 & 1
\end{array}\right] \quad L_{12}=\left[\begin{array}{cc}
1 & \frac{1-\epsilon}{2} \\
0 & \epsilon
\end{array}\right] \quad L_{13}=\left[\begin{array}{cc}
\epsilon & \epsilon \frac{1-\epsilon}{2} \\
\frac{1-\epsilon}{2} & \left(\frac{1+\epsilon}{2}\right)^{2}
\end{array}\right]
$$

The maps $F_{11}$ and $F_{21}$ correspond to avalanches of size 0 , the maps $F_{12}$ and $F_{22}$ correspond to avalanches of size 1 , and the maps $F_{13}$ and $F_{23}$ correspond to avalanches of size 2 .

It was discovered in $\mathrm{BCK}$ that the physical attractor $\mathcal{Y}$ has the following simple structure:

$$
\mathcal{Y}=\left\{\left(\frac{1+\epsilon}{1-\epsilon}, \frac{\epsilon}{2-\epsilon}\right),\left(\frac{\epsilon}{2-\epsilon}, \frac{1+\epsilon}{1-\epsilon}\right),\left(\frac{1+\epsilon}{1-\epsilon}, \frac{1+\epsilon}{1-\epsilon}\right)\right\}
$$

for $E_{c} \in\left[\frac{1+\epsilon}{1-\epsilon}, \frac{2}{1-\epsilon}\right]$ We denote these points by $a, b, c$ so that $\mathcal{Y}=\{a, b, c\}$. The maps $F_{1} \mid \mathcal{Y}$ and $F_{2} \mid \mathcal{Y}$ are permutations of $\mathcal{Y}$ :

$$
F_{1}\left|\mathcal{Y}=\left(\begin{array}{lll}
a & b & c \\
b & c & a
\end{array}\right), F_{2}\right| \mathcal{Y}=\left(\begin{array}{lll}
a & b & c \\
c & a & b
\end{array}\right)
$$

Figure 6 shows the physical attractor $\mathcal{Y}$ and the maps $\left.F_{1}\right|_{Y}$ and $\left.F_{2}\right|_{Y}$ for $E_{c}=7 / 2$ and $\epsilon=1 / 2$. We construct a partition $\mathcal{R}=\left\{R_{1}, \ldots, R_{6}\right\}$ of $\Sigma_{N}^{+} \times Y$ by

$$
\begin{array}{lll}
R_{1}=[1] \times\{a\} & R_{2}=[1] \times\{b\} & R_{3}=[1] \times\{c\} \\
R_{4}=[2] \times\{a\} & R_{5}=[2] \times\{b\} & R_{6}=[2] \times\{c\}
\end{array}
$$

We let $A=\left\|a_{i j}\right\|$ be the $6 \times 6$ matrix where $a_{i j}=1$ if $F\left(R_{i}\right) \cap R_{j} \neq \emptyset$ and 
$a_{i j}=0$ otherwise. It is easy to verify that

$$
A=\left[\begin{array}{llllll}
0 & 1 & 0 & 0 & 1 & 0 \\
0 & 0 & 1 & 0 & 0 & 1 \\
1 & 0 & 0 & 1 & 0 & 0 \\
0 & 0 & 1 & 0 & 0 & 1 \\
1 & 0 & 0 & 1 & 0 & 0 \\
0 & 1 & 0 & 0 & 1 & 0
\end{array}\right]
$$

It is clear that $g_{\mathcal{R}}: \mathcal{A} \rightarrow \Sigma_{A}^{+}$is a topological conjugancy of the maps $\left.F\right|_{\mathcal{A}}$ and $\sigma_{A}^{+}$. The matrix $A$ is transitive and $\operatorname{Sp}(A)=\{-1,-1,0,0,0,2\}$. Hence $h_{\text {top }}\left(\left.F\right|_{\mathcal{A}}\right)=\log 2$.

If $\mu$ is the SRB-measure on $\mathcal{A}$, with $\left(\pi_{u}\right)_{*} \mu$ being the uniform Bernoulli measure on $\Sigma_{N}^{+}$, then $\left(g_{\mathcal{R}}\right)_{*} \mu$ is the Perry measure on $\Sigma_{A}^{+}$. With respect to this measure it is easy to see that the average avalanche size is $\bar{s}_{0}=1$. It then follows from that the sum of the negative Lyapunov-exponents is $\chi_{1}+\chi_{2}=\log \epsilon$. For instance we see that for $E_{c}=11 / 2$ and $\epsilon=2 / 3$ we have $h_{\mu}\left(\left.F\right|_{\mathcal{A}}\right)>\left|\chi_{1}\right|+\left|\chi_{2}\right|$. So even though $F_{1} \mid \mathcal{Y}$ and $F_{2} \mid \mathcal{Y}$ are invertible, the Ruelle inequality (and therefore the Pesin formula) cannot be reversed (though this was argued in [BCK]).

We also remark that for $E_{c}=(1+\epsilon) /(1-\epsilon)$ we have $\mathcal{Y} \subset S(F)$, so the standard construction of SRB-measure will fail in this case. Still there is clearly a natural invariant measure.

The example with a trivial physical attractor can be generalized to all $N$ for $d=1$. Then $\mathcal{Y}$ consists of $N+1$ points $z_{0}, z_{1}, \ldots, z_{N}$ given by

$$
z_{n}=\frac{1+\epsilon}{1-\epsilon}(1,1, \ldots, 1)-e_{n}
$$

where $e_{0}=0$ and $e_{1}, \ldots, e_{N}$ is the standard basis in $\mathbb{R}^{N}$.

Example B: For $E_{c}=\epsilon=1 / 3$ the dynamics is very simple. The map $F_{1}$ has two domains of continuity: $M_{11}=\{(x, y) \in M \mid y \leq-2 x / 3+1 / 3\}$ and $M_{12}=M \backslash M_{11}$. The domains of continuity for $F_{2}$ are given by symmetry. The maps are given by $F_{11}(x)=L_{11}\left(x+e_{1}\right)$ and $F_{12}(x)=L_{12}\left(x+e_{1}\right)$, where

$$
L_{11}=\frac{1}{9}\left[\begin{array}{ll}
2 & 3 \\
2 & 3
\end{array}\right] \text { and } L_{12}=\frac{2}{27}\left[\begin{array}{ll}
2 & 3 \\
2 & 3
\end{array}\right] .
$$

We see that all four $F_{i j}$ are mappings to the diagonal line in $M$. In fact, $F_{11}\left(M_{11}\right)=F_{21}\left(M_{21}\right)=\left[p_{1}, p_{2}\right]$, where $p_{1}=(2 / 9,2 / 9)$ and $p_{2}=(1 / 3,1 / 3)$. The interval is contained in $M_{12} \cap M_{22}$ since the two lines of singularity intersect the diagonal in the point $(1 / 5,1 / 5)$. The images of $M_{12}$ and $M_{22}$ also coincide and is an interval $\left[p_{1}, p_{3}\right] \subset\left[p_{1}, p_{2}\right]$, where $p_{3}=(22 / 81,22 / 81)$. This shows that singularities are removable after one iteration. It is easy to see that for all $\mathbf{t} \in \Sigma_{N}^{+}$the dynamics will contract to the fixed point $P=(4 / 17,4 / 17)$. Hence the attractor of the system is $\mathcal{A}=\Sigma_{N}^{+} \times\{P\}$. The dynamics on the attractor is of course conjugated to $\sigma_{N}^{+}$. 


\section{Boris Kruglikov, Martin Rypdal}



Figure 7: The region in the $\left(\epsilon, E_{c}\right)$-plane where the avalanches are the same as for $E_{c}=\epsilon=1 / 3$. The point $(1 / 3,1 / 3)$ is shown in the interior of the region.

The example can easily be extended to a neighborhood of $(1 / 3,1 / 3)$ in the $\left(\epsilon, E_{c}\right)$-plane. In the region

$$
\max \left\{\frac{1-2 \epsilon+13 \epsilon^{2}}{5+12 \epsilon-13 \epsilon^{2}}, \frac{\epsilon\left(7 \epsilon^{2}-6 \epsilon+3\right)}{7 \epsilon^{3}-10 \epsilon^{2}+7 \epsilon-4}\right\} \leq E_{c} \leq \min \left\{\frac{\epsilon}{1-\epsilon}, \frac{1-2 \epsilon+5 \epsilon^{2}}{1+4 \epsilon-5 \epsilon^{2}}\right\}
$$

we have the same avalanches as for $E_{c}=\epsilon=1 / 3$. This region is shown in Figure 7 For all points in the region the maps depend continuously on $\epsilon$ and the lines of singularity depend continuously on $\epsilon$ and $E_{c}$. The condition for an atomic spacial attractor is that the images of the domains do not intersect the singularities. For $E_{c}=\epsilon=1 / 3$ the images are bounded away from the lines of singularity, and hence there exists an open neighborhood of $(1 / 3,1 / 3)$ in the $\left(\epsilon, E_{c}\right)$-plane where the same holds, i.e. the attractor is of the form $\Sigma_{N}^{+} \times\{P\}$ (where $P$ is a point in $M$ ) and the dynamics is conjugated to $\sigma_{N}^{+}$.

Example C: Let us consider $E_{c}=1 / 3$ and $\epsilon=1 / 2$. In this case there are 28 domains of continuity and 28 corresponding maps. A computer program is written to compute the sets $U_{n}$. The program uses exact calculations of the edges of the polygons that make up $U_{n}$, and hence it can be used to give rigorous "proof by computer" of removability of singularities. By using the program we obtain that singularities are removable. In fact $U_{5} \cap \mathcal{S}=\emptyset$. The set $U_{5}$ consists of 13 connected components. Figure 8 shows the set $U_{5}$ and the lines of singularity, and Figure 9 is a schematic illustration of how these connected components are situated with respect to the lines of singularity. The intersection of the connected components of $U_{5}$ with $\mathcal{Y}$ are denoted by $Y_{1}, \ldots, Y_{13}$. Then we construct the partition $\mathcal{R}=\left\{[i] \times Y_{j}\right\}$, and enumerate the 


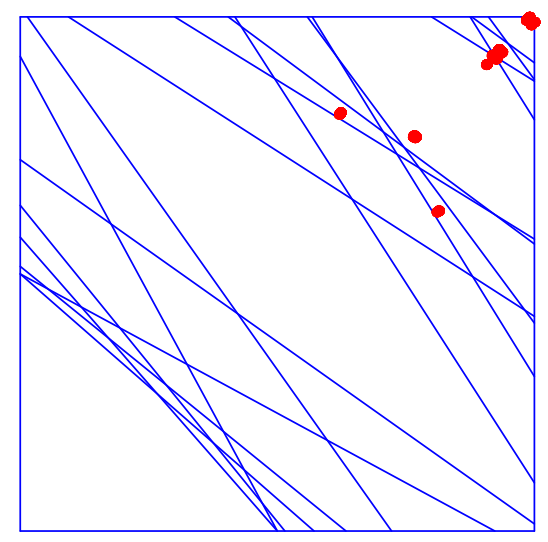

Figure 8: Shows the set $U_{5}$ for $N=2, E_{c}=1 / 3$ and $\epsilon=1 / 2$. The points on the attractor are magnified in order to make them visible in the figure, and hence it looks as if they intersect singularities, but in fact they do not.

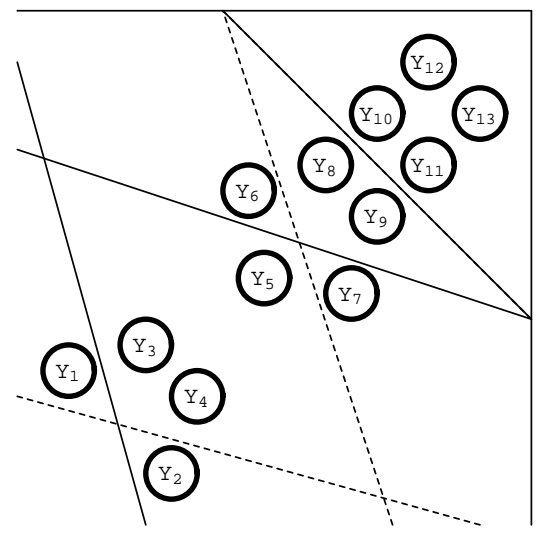

Figure 9: Shows how the 13 spatial partition elements are situated with respect to the lines of singularity. 


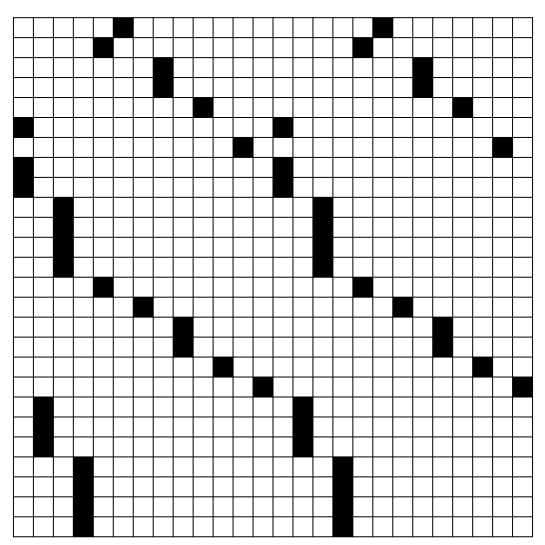

Figure 10: Shows the matrix $A$ for the coding of Example C. Black squares are 1-s and white squares are $0-$ s.

elements so that $\mathcal{R}=\left\{R_{1}, \ldots, R_{26}\right\}$, where

$$
\begin{aligned}
& R_{1}=[1] \times Y_{1} \quad R_{3}=[1] \times Y_{2} \quad \ldots \quad R_{25}=[1] \times Y_{13} \\
& R_{2}=[2] \times Y_{1} \quad R_{4}=[2] \times Y_{2} \quad \ldots \quad R_{26}=[2] \times Y_{13} .
\end{aligned}
$$

We construct the $26 \times 26$ matrix $A=\left\|a_{i j}\right\|$ by letting $a_{i j}=1$ if $F\left(R_{i}\right) \cap R_{j} \neq \emptyset$, and $a_{i j}=0$ otherwise. After making the computations, the matrix $A$ becomes as shown in Figure 10 The black squares represent ones and white squares represent zeros. Direct computation shows that the matrix $A$ is transitive.

Since singularities are removable the map $g_{\mathcal{R}}: \mathcal{A} \rightarrow \Sigma_{\mathcal{A}}^{+}$is an avalanche conjugancy between $F_{\mathcal{A}}$ and $\sigma_{A}^{+}$. The SRB-measure projects to the Perry measure on $\Sigma_{A}^{+}$, so it is possible to calculate properties such as average avalanche size. In this example a computation gives $\bar{s}_{0}=123 / 17$. The spectral radius of the matrix $A$ is 2 , and hence $h_{\text {top }}\left(\sigma_{A}^{+}\right)=\log 2$.

Example D: In the previous examples singularities are removable. This is however not always the case. Figure 11] shows the set $U_{20}$ for $N=2, E_{c}=7$ and $\epsilon=1 / 2$. This is an example where singularities are non-removable. We will in the following show that singularities are non-removable for $E_{c}=(3+\epsilon) /(1-\epsilon)$.

Since $(3+\epsilon) /(1-\epsilon)>(1+\epsilon) /(1-\epsilon)$, the domains of continuity are given by the formulas presented in Example A. Take the point $p=\left(E_{c}, E_{c}-1\right) \in M_{13}$. See Figure12 Observe that $p$ lies on the horizontal line $x_{1}=E_{c}-1$ and hence $p \in S(F)$. Clearly $p$ is in the interior of $M_{13}$, so

$$
F_{1}(p)=F_{13}\left(\frac{3+\epsilon}{1-\epsilon}, \frac{3+\epsilon}{1-\epsilon}-1\right)=\left(\frac{3+\epsilon}{1-\epsilon}-1, \frac{3+\epsilon}{1-\epsilon}-2\right)=p-(1,2) .
$$

Denote $q_{1}:=F_{1}(p)$ and observe the it lies on the singularity line $x_{2}=E_{c}-1$. On the other hand $q_{1}$ is in the interior of $M_{21}$, so $F_{2}\left(q_{1}\right)=p-(1,1)$. Denote 


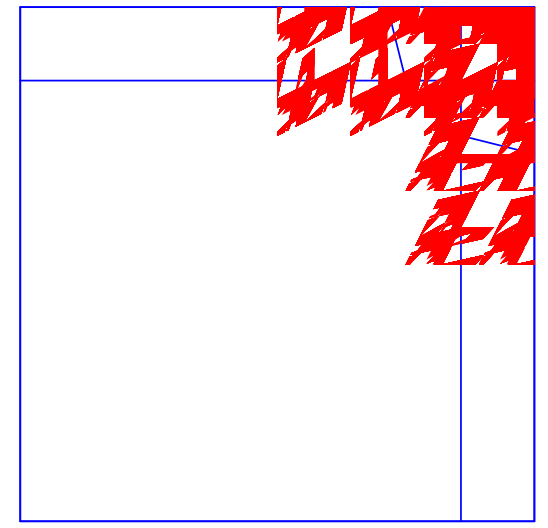

Figure 11: Shows the set $U_{20}$ for $E_{c}=7$ and $\epsilon=1 / 2$. In this example singularities are non-removable.

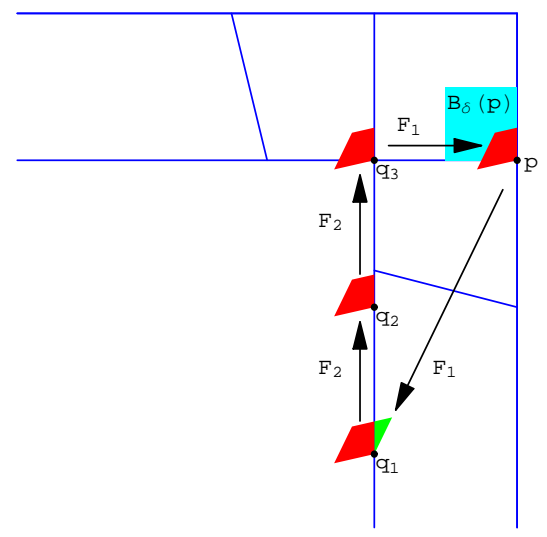

Figure 12: Illustration of the fact that singularities are non-removable for $E_{c}=7$ and $\epsilon=1 / 2$. 
$q_{2}:=F_{2}\left(q_{1}\right)$. This point also lies in the interior of $M_{21}$. Let $q_{3}:=F_{2}\left(q_{2}\right)=$ $p-(1,0)$. It is clear that $F_{11}\left(q_{3}\right)=p$, and in this sense $p$ is a periodic point. However, $F_{1}\left(q_{3}\right)$ is not well defined since $q_{3} \in \partial M_{12} \cap \partial M_{13}$.

In the following we let $\left\langle z_{1}, \ldots, z_{n}\right\rangle$ denote the open convex polygon with edges $z_{1}, \ldots, z_{n}$. Define $B_{\delta}(p)=\langle p, p+(0, \delta), p+(-\delta, \delta), p+(-\delta, 0)\rangle$. For small $\delta>0$ we have $B_{\delta}(p) \in M_{13}$, and hence

$$
F_{1}\left(B_{\delta}(p)\right)=F_{13}\left(B_{\delta}(p)\right)=\left\langle q_{1}, q_{1}+\delta a, q_{1}+\delta b, q_{1}+\delta c\right\rangle,
$$

where

$$
a=\left(\frac{1-\epsilon}{2}, \epsilon\right), b=\left(\frac{1-4 \epsilon-\epsilon^{2}}{4}, \frac{1+\epsilon}{2} \epsilon\right), c=\left(-\left(\frac{1+\epsilon}{2}\right)^{2},-\frac{1-\epsilon}{2} \epsilon\right) .
$$

The polygon $F_{1}\left(B_{\delta}(p)\right)$ intersects the singularity line $x_{1}=E_{c}-1$. See Figure [12. A simple computation shows that

$$
F_{1}\left(B_{\delta}(p)\right) \cap M_{11}=\left\langle q_{1}, q_{1}+\delta a^{\prime}, q_{1}+\delta b, q_{1}+\delta c\right\rangle,
$$

where

$$
a^{\prime}=\left(0,\left(\frac{2 \epsilon}{1+\epsilon}\right)^{2}\right)
$$

It then follows from the above discussion that

$$
F_{1} \circ F_{2}^{2}\left(F_{1}\left(B_{\delta}(p)\right) \cap M_{11}\right)=\left\langle p, p+\delta a^{\prime}, p+\delta b, p+\delta c\right\rangle .
$$

It is then easy to verify that for all $\delta>0$ there is $\gamma>0$ such that $B_{\gamma}(p) \subset$ $\pi_{s} \circ F^{4}\left(\Sigma_{N}^{+} \times B_{\delta}(p)\right)$. So for each $n \in \mathbb{N}$ there is $\delta_{n}>0$ with $B_{\delta_{n}}(p) \subset U_{n}$. The image of $B_{\delta_{n}}(p)$ under $F_{1}$ intersects singularities, and its closure contains the point $q_{1}$, which thus is an essential singularity. Clearly the points $p, q_{2}$ and $q_{3}$ are also essential singularities.

\section{Statistical properties}

In order to evaluate the entropy and Lyapunov spectrum of the physical model in the thermodynamic limit we need to derive several estimates for the asymptotic behavior of observables like avalanche size, avalanche duration and "waitingtime" between avalanches. The results are derived using only the uniform Bernoulli measure on $\Sigma_{N}^{+}$, and hence hold for any SRB-measure and for timeaverages.

In 77.3 we define the thermodynamic limit as the double limit $E_{c} \rightarrow \infty$, $L=\sqrt[d]{N} \rightarrow \infty$, contrary to [BCK], where the thermodynamic limit is defined as the limit $L \rightarrow \infty$ only. This is important as the quasi-classical limit since equivalently means a fixed energy, but the energy quantum of Section $1 \delta \rightarrow 0$. As $E_{c} \rightarrow \infty$ we must make a scaling of time in the physical model. Otherwise the influx of energy to the system will go to zero. With this new scaling we show that the entropy goes to zero and the Lyapunov spectrum is collapsing.

We do not provide strict mathematical proofs, but still think important to include the discussion of our results from the physical point of view. 
Dynamics and entropy of SOC

\subsection{Statistics of observables}

Let $\tau$ be the coordinate measuring the duration of avalanche and let $\omega$ correspond to the interval between avalanches (minimal value 1). We will also study the observable $s$ - the avalanche size (defined in 1.3). While in the first case we consider only actual avalanches, so that $\tau>0$, in the second we make distinction between $s_{0}$ - all avalanches including the trivial case of under-critical state $(s=0)$ and $s_{+}-$the actual avalanches, so that $s_{+}>0$.

The reason for introducing two different avalanche size observables is the following: $s_{0}$ plays a crucial role in mathematical investigation of the model (see 97.4 , while $s_{+}$is important from physical perspective. In 17.3 we will see that physical observables should allow a thermodynamic limit.

Denote by $\bar{\tau}, \bar{\omega}, \bar{s}_{0}, \bar{s}_{+}$the corresponding mean time-average quantities, each of which is a function on the space-factor $M$ and is defined as follows:

$$
\bar{\sigma}(x)=\int_{\Sigma_{N}^{+}} \lim _{k \rightarrow \infty} \frac{1}{k} \sum_{i=0}^{k-1} \sigma\left(\hat{f}^{i}(\mathbf{t}, x)\right) d \mu_{\mathrm{Ber}}
$$

with $\mu_{\text {Ber }}$ being the Bernoulli measure on the one-sided shifts (by Birkhoff ergodic theorem the time-average limit exists almost everywhere and is measurable). This function is invariant in the sense: $\bar{\sigma}(x)=\frac{1}{N} \sum_{i=1}^{N} \bar{\sigma}(\hat{f}(i, x))$.

Whenever the system is ergodic with respect to an invariant measure $\mu$ the function $\bar{\sigma}$ is constant $\mu$-a.e. and equals the space (ensemble) average

$$
\langle\sigma\rangle_{\mu}=\int_{\Sigma_{N}^{+} \times M} \sigma d \mu .
$$

If the system has a unique invariant SRB-measure the function $\bar{\sigma}$ is constant $\mu_{\mathrm{Ber}} \times \mu_{\mathrm{Leb}}-$ a.e. and equals the space (ensemble) average

$$
\langle\sigma\rangle=\int_{\Sigma_{N}^{+} \times M} \sigma d \mu_{\mathrm{SRB}} .
$$

We will need the maximal values of these observables in a sequel, which we denote by $\tau_{\max }, \omega_{\max }$ and $s_{\max }=\max s_{0}=\max s_{+}$respectively. We shall calculate their asymptotics in $L$ and $E_{c}$.

Denote by $\varphi_{1} \sim \varphi_{2}$ the asymptotic equivalence relation meaning that the ratio $\varphi_{1} / \varphi_{2}$ has subexponential grow/decay. We denote the equivalence by $\approx$, when the limit of the ratio is 1 .

Lemma 21. For $E_{c} \gg 1$ the maximal avalanche time and size have the asymptotics: $\tau_{\max } \sim L^{\gamma_{\tau}}, \omega_{\max }=L^{d} E_{c}, s_{\max } \sim L^{d+\gamma_{s}}$, where $\gamma_{\tau}=\gamma_{s}=1$ for $d=1$ and $1<\gamma_{\tau}, \gamma_{s}<d$ for $d>1$.

Proof. Consider at first the simple case $d=1$. The maximum avalanche duration and size are achieved when all sites contribute to the avalanche, i.e. 


\section{Boris Kruglikov, Martin Rypdal}

their energies are sufficiently big and there is one site with energy greater than $E_{c}-1$ to initiate the avalanche. Actually, if some site has small energy, it will serve as a boundary and the avalanche wave reflects from it (to be explained below).

Assuming $E_{c}>\frac{\epsilon}{1-\epsilon}$ we know from Lemma [ that in the avalanche process each site, whenever overcritical, relaxes until in the next step it receives a sufficient portion of energy to become overcritical and relax etc. In other words, the sites blink, being under- and over-critical in turn. But in this process they make overcritical their neighbors and the process propagates as a wave, with only difference that its front excites new sites, while in the traversed region there remain blinking overcritical sites.

This wave spreads along the interval $B_{L}^{1}=[1, L] \subset \mathbb{Z}$ towards its boundary and then it reflects from it, bearing now relaxation. In fact, as the front wave reaches the boundary it losses a substantial part of the energy on the boundary sites, which thus cannot be recovered and remain undercritical for the rest of this avalanche. They influence their neighbors to stop being critical and so forth. Thus we obtain the reflected wave that, in contrast with the first one, turns overcritical sites into relaxed. When the wave hits itself, the avalanche process stops.

It is clear that the duration of this avalanche is $\tau_{\max } \approx L$. The number of involved sites corresponds to the area of the triangle with a side $B_{L}^{1}=[1, L]$ and height $L / 2$ (recall that each site is overcritical only half time of its blinking period), whence $s_{\max } \approx L^{2} / 4$.

Consider now the case of dimension $d>1$. Here the scenario of maximal avalanche is more complicated and consists of three stages (the proof is similar to the case $d=1$, but quite lengthy and will be suppressed). Again the maximum avalanche duration and size are achieved when all sites contribute to the avalanche, though now if some isolated site has smaller energy it serves as a boundary only once but then on the next several waves it receives the required portion of energy and follows the general scheme of motion.

At the first stage a site is excited and it initiates the rhombus-shape wave (a cube in the Manhattan metric, see Figure 13) that spreads to the boundary of the cube $B_{L}^{d}=[1, L]^{d} \subset \mathbb{Z}^{d}$ (in time $\approx L / 2$ if the center of the rhombus is placed near the center of the cube). The second stage begins as the wave reaches the boundary face and reflects from it (See Figure 14). The reflected wave is almost momentary overthrown by the coming overcritical wave, which again reflects from the boundary, come now deeper into the interior of the cube, but is overthrown too etc. If one looks along the boundary face, the reflected wave travel along towards a vertex with preserved form (like a soliton) and then disappears into this vertex (there occur strong interactions with other waves in this corner). But if one looks into the perpendicular direction, the collection of reflected waves oscillates (each reflected wave enters deeper and deeper into the cube) contributing to the avalanche duration the sum $1+2+3+\ldots$, which stops with the end of the second stage (we do not specify the sum precisely because after some oscillations the wave front becomes more and more eroded by the interactions between overcritical and relaxing waves; this impairs the sum and 
Dynamics and entropy of SOC

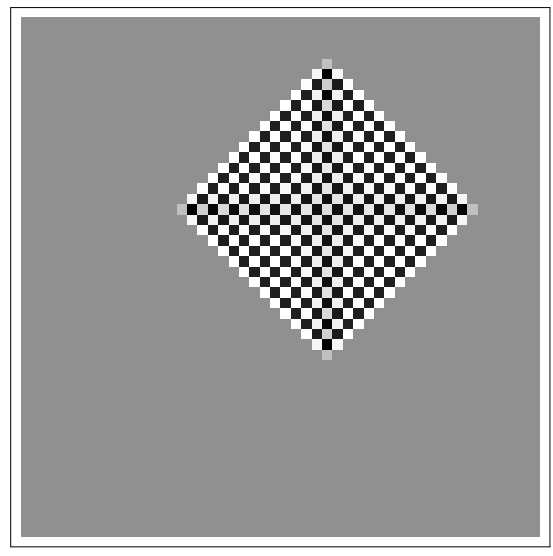

Figure 13: The picture is a "snapshot" of the lattice $\Lambda$ for $d=2, L=10, E_{c}=7$ and $\epsilon=1 / 2$. A single site in the marginally stable configuration has been exited, and a great avalanche is unfolding in a rhombus shape. This is the first stage of this avalanche. The white squares are overcritical sites, the gray squares are sites with energy just bellow $E_{c}$ and the black squares have energy approximately equal $\epsilon E_{c}$.

decreases the exponent, but not too drastically).

The third stage begins as the main body of the overcritical sites becomes disconnected and the avalanches behaves like worms crawling along the high energy fractal-like collection of states. We illustrate this in Figure 15] From the description of the maximal avalanche process it is clear that the asymptotic exponents $\gamma_{\tau}, \gamma_{s}$ do not depend on the energy $E_{c} \gg 1$. Let us denote (the sequences are increasing, so the limits exist):

$$
\gamma_{\tau}=\lim _{L \rightarrow \infty} \frac{\log \tau_{\max }}{\log L} \quad \text { and } \quad d+\gamma_{s}=\lim _{L \rightarrow \infty} \frac{\log s_{\max }}{\log L} .
$$

Duration of the first stage of avalanche is $\sim L$. The above arguments show that the exponent $\gamma_{\tau}^{\prime}$ of the second stage is $>1$. The last stage can only increase it: $\gamma_{\tau} \geq \gamma_{\tau}^{\prime}$. To see that $\gamma_{\tau}<d$ we note that in average the number of critical sites on the boundary is about $\kappa_{L} \sim L^{\gamma_{\kappa}}$ with $0<\gamma_{\kappa}<d$. Thus we have a constant flow of energy out of the system with the average speed $>\frac{1-\epsilon}{2 d} \kappa_{L} E_{c}$, and the inequality $E_{\mathrm{tot}} \leq L^{d} E_{c}$ proves the claim.

To estimate the maximal avalanche size exponent $\gamma_{s}$ consider again the second stage of the above scenario. The number of involved sites corresponds to the volume of the prism $\Pi_{L}$ over the cube $B_{L}^{d}$ with height $\ell \approx \frac{1}{2} \tau_{\max } \sim L^{\gamma_{\tau}^{\prime}}$, i.e.

$$
L^{d+\gamma_{s}^{\prime}} \sim \operatorname{Vol}_{d+1}\left(\Pi_{L}\right)=\frac{\ell}{d+1} \cdot \operatorname{Vol}_{d}\left(B_{L}^{d}\right) \sim L^{d+\gamma_{\tau}^{\prime}} .
$$

Thus $\gamma_{s} \geq \gamma_{s}^{\prime}=\gamma_{\tau}^{\prime}>1$. Inequality $\gamma_{s}<d$ follows from the inequality from above for the duration exponent $\gamma_{\tau}$.

The maximal value of the waiting time is obvious. 


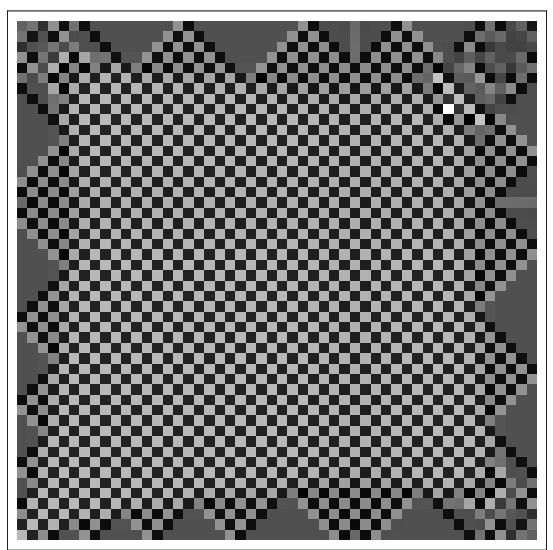

Figure 14: The picture shows a "snapshot" of the second stage of the avalanche shown in Figure 13 We can see the well-shaped (soliton-like) waves of energy near the boundary and observe how their form begins being eroded near the vertices.

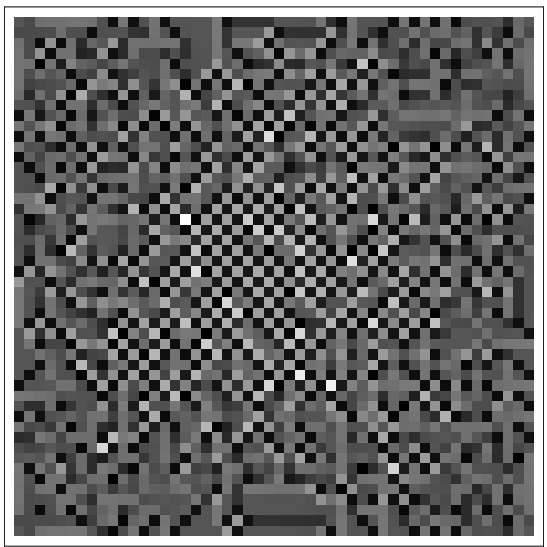

Figure 15: The picture shows a "snapshot" of the third stage of the avalanche shown in Figure 13 The energy configuration has a fractal structure where the avalanche can sustain in regions of high energy. 
Dynamics and entropy of SOC

\subsection{Asymptotic of the statistical data}

Now we can study statistics of the avalanche data asymptotically (as for the thermodynamic limit).

We will need the following technical statement (informal only for $E_{c}>1$ ):

Lemma 22. Almost every (w.r.t. a random excitation sequence) spatial trajectory returns to the cube $B_{0}=\left(E_{c}-1, E_{c}\right]^{N}$.

Proof. It follows from Proposition 4 that $K=\left\{x \in M \mid \exists i: x_{i} \in\left(E_{c}-1 ; E_{c}\right]\right\}$ is a return set, i.e. every trajectory $F^{n}(\mathbf{t}, x)$ meets $\Sigma_{N}^{+} \times K$. Partition the spatial part $\mathcal{Y}$ of the attractor according to the hyperplanes collection $\mathcal{H}=$ $\cup_{i, m \in \mathbb{N}}\left\{x_{i}=E_{c}-m\right\}$.

Each such a part can be shifted by an excitation sequence to the cube $B_{0}$. The probability of all such sequences (where the avalanche starts from the set $B_{0}$ of maximal energy in all sites) is positive. Let $\rho>0$ denote the minimum of these probabilities over the finite set of all partition elements of $Y$ by $\mathcal{H}$. Then the probability of not entering $B_{0}$ in $k$ successive avalanches is less than $(1-\rho)^{k}$.

Therefore since the number of avalanches tend to infinity as we iterate the dynamics, the measure of trajectories staying away from $B_{0}$ is zero.

Theorem 23. We have the following asymptotic estimates valid as $E_{c} \rightarrow \infty$ (and $N \gg 1$ fixed) or $L=\sqrt[d]{N} \rightarrow \infty$ (and $E_{c} \gg 1$ fixed):

(i) $\bar{\tau} \sim L^{\gamma_{\tau}}$;

(ii) $\bar{\omega} \sim E_{c}$;

(iii) $\bar{s}_{0} \sim L^{d+\gamma_{s}} / E_{c}$;

(iv) $\bar{s}_{+} \sim L^{d+\gamma_{s}}$,

where $\gamma_{\tau}, \gamma_{s}$ are the same exponents as in Lemma21 (thus $\gamma_{\omega}=\lim _{L \rightarrow \infty} \frac{\log \bar{\omega}}{\log L}=0$ ).

Proof. The maximal avalanche size is achieved for a certain configuration of states $V \subset M$, which we can bound as follows: $U_{1} \subset V \subset U_{d}$, where

$$
U_{j}=\left\{x \in M \mid \forall i:\left(1-j \frac{1-\epsilon}{2 d}\right) E_{c}<x_{i} \leq E_{c} \text { and } \exists i_{0}: x_{i_{0}}>E_{c}-1\right\} .
$$

Denote also $\tilde{U}_{j}=\left\{x \in M \mid \forall i:\left(1-j \frac{1-\epsilon}{2 d}\right) E_{c}<x_{i} \leq E_{c}\right\} \supset U_{j}$.

To estimate the measure of the sites leading to the maximal avalanche we consider preimages of $\Sigma_{N}^{+} \times \tilde{U}_{j}$ under the map $F$. It is clear that one needs $k_{\epsilon} \in\left[\frac{2}{1-\epsilon}, \frac{2 d}{1-\epsilon}\right]$ different backwards iterations $F^{-i_{s}}, s=1, \ldots, k_{\epsilon}$, to cover the spatial attractor $\mathcal{Y}$. Since the measure $\mu$ is $F$-invariant, we get for its $\pi_{s}$-pushforward: $\mu_{s}\left(\tilde{U}_{j}\right) \approx \rho_{j} / k_{\epsilon}$, which is $E_{c}$-independent. Thus we get the same exponent $\left(d+\gamma_{s}\right)$ for $\bar{s}_{+}$as for $s_{\max }$.

The same arguments yield the asymptotic of $\bar{\tau}$.

To obtain the asymptotic of $\bar{\omega}$ in $L$ we note that since the amount of lost energy is $<C L^{\gamma_{\kappa}+\delta} E_{c}$ (where $\gamma_{\kappa}<d$ is the quantity from the proof of Lemma 

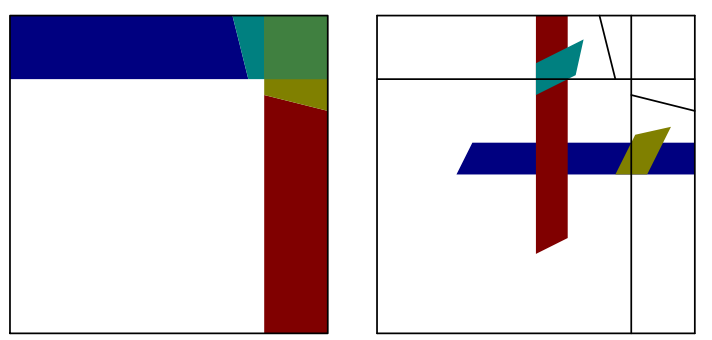

Figure 16: The figure illustrates how the continuity domains are mapped under $F_{1}$ and $F_{2}$ for $N=2$ and $E_{c}>\epsilon /(1-\epsilon)$.
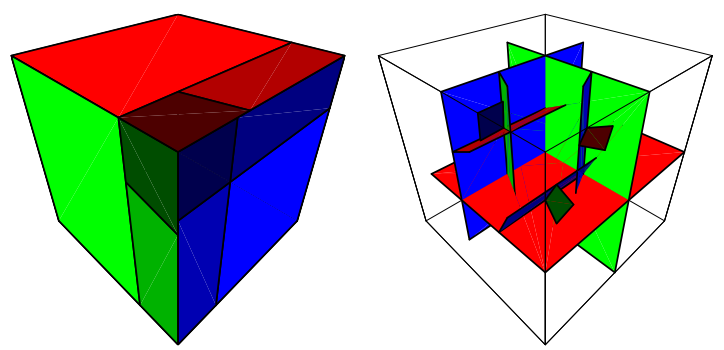

Figure 17: The figure illustrates how the faces of the continuity domains are mapped under $F_{1}, F_{2}$ and $F_{3}$ for $N=3$ and $E_{c}>\epsilon /(1-\epsilon)$. The point of view is at $\left(E_{c}, E_{c}, E_{c}\right)$.

211), the average remained energy in a site of configuration obtained from a maximal one after an avalanche is $\left(L^{d} E_{c}-C L^{\gamma_{\kappa}+\delta} E_{c}\right) / L^{d} \approx E_{c}$. Thus the waiting time does not grow with $L$.

The asymptotic of $\bar{\omega}$ in $E_{c}$ is quite different: If $N$ is fixed but $E_{c}$ grows, then any state from $\partial M$ becomes at distance $\theta_{N} \cdot E_{c}$ after some relatively small number of iterations.

Let us first demonstrate the idea in the simple case $N=2$. For critical energy $E_{c}>\epsilon /(1-\epsilon)$ the picture of avalanches is shown on Figure [16 We see that in a few steps of the dynamics the configuration becomes far from $\partial M$, i.e. it strongly contracts in all directions. Actually, it is possible to imagine the situations when the point is mapped to the vertical strip and then is shifted horizontally for a long time by excitations of the first site, but probability of this event exponentially goes to zero as $E_{c} \rightarrow \infty$. Thus in a relatively short time the point from $\partial M$ is mapped into the square $\left[0, \theta_{2} E_{c}\right]^{2}$, where the constant $\theta_{2}$ is $E_{c}$-independent. To achieve the boundary $\partial M$ again it needs $\sim\left(1-\theta_{2}\right) 2 E_{c}$ random excitations.

The similar picture happens for $N=3$, see Figure 17 In the general case Theorem 5 insures that after some (few) number of steps we get strong con- 
Dynamics and entropy of SOC

traction, so that the point becomes in the cube $\left[0, \theta_{N} E_{c}\right]^{N}$, i.e. far from the boundary $\partial M$. Thus we need $\sim\left(1-\theta_{N}\right) N E_{c}$ excitations to make it overcritical and this implies the claim. Note that the asymptotic for $\bar{\omega}$ is not for the double limit $E_{c} \rightarrow \infty, L \rightarrow \infty$, but for two partial limits only.

To obtain the estimate for $\bar{s}_{0}$ we need to estimate the conditional measure $\mu_{s}\left(U_{j} \mid \tilde{U}_{j}\right)$, which coincides with the probability that a randomly chosen configuration $x \in \tilde{U}_{j}$ and site $i$ satisfy: $x_{i}>E_{c}-1$. This probability is $\approx b_{1} / E_{c}$. Thus $\mu\left(\Sigma_{N}^{+} \times V\right) \sim \sigma_{\epsilon} / E_{c}$ and the mean avalanche size is $\left\langle s_{0}\right\rangle \sim L^{d+\gamma_{s}} / E_{c}$.

This is however the space-average (the arguments below work also for $\bar{s}_{+}$, $\bar{\tau})$. We would obtain the same for the time-average of Lebesgue a.e.-initial condition if we have an SRB-measure, or for $\mu$-a.e. if we have an ergodic measure $\mu$. But we cannot guarantee existence of an SRB-measure or an ergodic measure. However, for a non-ergodic measure, we can decompose $\mathcal{A}$ into ergodic components, where the Birkhoff theorem works. By Lemma 22 each ergodic component (with non-trivial contribution) intersects in its spatial part the topenergy cube $B_{0}$ and so for each of it the same asymptotic of space-averages holds with universal exponents but maybe different coefficients. Therefore we obtain the required asymptotic for $\bar{s}_{0}$.

Another way to get the last asymptotic is via the formula $\bar{s}_{0}=\frac{\bar{\omega} \cdot 0+1 \cdot \bar{s}_{+}}{\bar{\omega}+1}$.

For $d=1, E_{c} \in\left[\frac{1+\epsilon}{1-\epsilon}, \frac{2}{1-\epsilon}\right]$ we already have $\bar{\tau} \sim N=L^{1}$ as the theorem states, but $\bar{s}_{0} \sim N=L^{1}, N \rightarrow \infty$, which shows in the last respect the critical energy $E_{c}=2 /(1-\epsilon)$ is small.

In [BCK] the estimate $\gamma_{\tau}>1$ was predicted for all $d$, while this is a feature of the cases $d>1$. In the latter cases the analytic calculation of exact values of exponents $\gamma_{\tau}, \gamma_{s}$ is a difficult problem.

Remark 9. The difference between cases $d=1$ and $d>1$ demonstrated in the theorem is known in the physical literature. The former case is usually considered as the trivial SOC-model.

\subsection{Thermodynamic limit}

By thermodynamic limit of an observable $\phi$ we understand the double limit

$$
[\phi]_{\infty}=\lim _{\substack{L \rightarrow \infty \\ E_{c} \rightarrow \infty}} \phi
$$

if it exists. It is assumed that for physical observables this limit exists. In BCK only limit $L \rightarrow \infty$ was considered, though then the value of energy $E_{c}$ could serve as an essential parameter, which is not desirable in the SOC-paradigm. However it was suggested there that consideration of $E_{c} \rightarrow \infty$ can be helpful.

As an example of non-physical observable we expose $s_{0}$ (in $\$ 7.1$ we called it mathematically relevant): The double limit does not exists because the repeated limits are different:

$$
0=\lim _{L \rightarrow \infty} \lim _{E_{c} \rightarrow \infty} \bar{s}_{0} \neq \lim _{E_{c} \rightarrow \infty} \lim _{L \rightarrow \infty} \bar{s}_{0}=+\infty .
$$




\section{Boris Kruglikov, Martin Rypdal}

But $s_{+}$and $\tau$ are good physical observables, for the thermodynamic limits exist:

$$
[\bar{\tau}]_{\infty}=\infty, \quad\left[\bar{s}_{+}\right]_{\infty}=\infty
$$

From 3.4 (use iteration arguments in the topological case) and we obtain:

$$
\left[h_{\text {top }}(\hat{f})\right]_{\infty}=\left[h_{\text {top }}(F) / \bar{\tau}\right]_{\infty}=0 \quad \text { and } \quad\left[h_{\text {top }}(F)\right]_{\infty}=\infty .
$$

But with $\omega$ the situation is different because the proof (rather than the vague statement of part (ii)) of Theorem [23] implies: $\lim _{L \rightarrow \infty} \lim _{E_{c} \rightarrow \infty} \bar{\omega}=\infty$, while $\lim _{E_{c} \rightarrow \infty} \lim _{L \rightarrow \infty} \bar{\omega}$ is finite. Since $\omega$ is definitely physically relevant observable one needs the following reparametrization: $\omega \mapsto \omega / E_{c}$, which corresponds to contraction of the waiting time via the following ansatz:

We let the energy quantum added at a unit time to the system equal $\delta=\hbar$ (instead of 1 as before), but speed up time in the waiting intervals respectively: $E_{c}=E_{0} / \hbar, \omega_{\text {new }}=\omega \hbar$. Then the thermodynamic limit (the space part $M$ can be quantized similarly via $L=[l / \hbar]$ with $l$ a finite length) corresponds to the quasi-classical limit $\hbar \rightarrow 0$.

The duration of avalanche was suppressed in our definition of dynamics to length one. So if we want to find the entropy of the physical system, where each step of avalanche has time-duration one, we should multiply it by the probability of dropping energy into the system. For every trajectory this equals $\frac{\bar{\omega}}{\bar{\omega}+\bar{\tau}}$. This ratio behaves differently as $L \rightarrow \infty$ or $E_{c} \rightarrow \infty$, so we need the reparametrization described above.

In 4.2 we calculated the entropy of the "return" Zhang model $h_{\text {top }}(F)=$ $\log N$ (this was proved almost surely, but even with the possible entropy growth for some exceptional parameters the thermodynamic limit below is unaltered). But after reparametrization it changes. Denoting by $h^{\text {Zhang }}$ the entropy of the reparametrized system we get:

$$
h_{\mu}^{\text {Zhang }}=h_{\mu^{u}}\left(\sigma_{N}^{+}\right) \cdot\left\langle\frac{\bar{\omega}_{\text {new }}}{\bar{\omega}_{\text {new }}+\bar{\tau}}\right\rangle, \quad h_{\text {top }}^{\text {Zhang }}=d \cdot \log L \cdot\left(\frac{\bar{\omega}_{\text {new }}}{\bar{\omega}_{\text {new }}+\bar{\tau}}\right)_{\max } .
$$

This implies:

$$
\left[h_{\mu}^{\text {Zhang }}\right]_{\infty}=\left[h_{\text {top }}^{\text {Zhang }}\right]_{\infty}=0 .
$$

Therefore the expanding property is lost in the thermodynamic limit for the original physical system, as was already noticed in $\mathrm{BCK}$ for a bit different situation.

Remark 10. Notice that in the reparametrized system $\bar{\omega}_{n e w} \ll \bar{\tau}$, which is counter-intuitive for certain SOC-examples (sandpile, earthquakes etc, where one expects $\bar{\omega} \gg \bar{\tau}$ ). This indicates that the Zhang system should be modified by introducing the local contraction of time depending on the avalanche size or speed. We will not consider such gradient-type models here. 
Dynamics and entropy of SOC

\subsection{Lyapunov spectrum}

In $\$ 3.3$ we showed that the Zhang model is hyperbolic with one positive exponent and the remainder of the spectrum negative. This hyperbolicity is lost in the thermodynamic limit.

Proposition 24. For $E_{c} \geq \epsilon /(1-\epsilon)$ we have $\sum_{i=1}^{N} \chi_{i}^{-}=\bar{s}_{0} \log \epsilon$.

Proof. We cannot ensure the existence of an SRB-measure, so both the Lyapunov exponents and $\bar{s}_{0}$ should be seen as functions on $M$. From the general theory of Lyapunov exponents we know that

$$
\sum_{i=1}^{N} \chi_{i}^{-}(\hat{x})=\lim _{n \rightarrow \infty} \frac{1}{n} \sum_{t=1}^{n-1} \log \operatorname{det} \mathcal{T}\left(F^{t} \hat{x}\right) .
$$

From Proposition 7 we know that the formula $\operatorname{det}\left(L_{i j}\right)=\epsilon^{s_{i j}}$ holds for $E_{c} \geq$ $\epsilon /(1-\epsilon)$. Hence

$$
\begin{aligned}
\sum_{i=1}^{N} \chi_{i}^{-}(x) & =\sum_{i=1}^{N} \int_{\Sigma_{N}^{+}} \chi_{i}^{-}(\mathbf{t}, x) d \mu_{\mathrm{Ber}} \\
& =\int_{\Sigma_{N}^{+}} \sum_{i=1}^{N} \chi_{i}^{-}(\mathbf{t}, x) d \mu_{\mathrm{Ber}} \\
& =\int_{\Sigma_{N}^{+}} \lim _{n \rightarrow \infty} \frac{1}{n} \sum_{t=0}^{n-1} \log (\operatorname{det} \mathcal{T})\left(F^{t}(\mathbf{t}, x)\right) d \mu_{\mathrm{Ber}} \\
& =\int_{\Sigma_{N}^{+}} \lim _{n \rightarrow \infty} \frac{1}{n} \sum_{t=0}^{n-1} s(\mathbf{t}, x) d \mu_{\mathrm{Ber}} \log \epsilon \\
& =\bar{s}_{0}(x) \log \epsilon .
\end{aligned}
$$

Corollary 4. $\left|\bar{\chi}^{-}\right|=\frac{\bar{s}_{0}}{N} \log \frac{1}{\epsilon} \rightarrow 0$ as $E_{c} \rightarrow \infty$, but $\left|\bar{\chi}^{-}\right| \rightarrow \infty$ as $L \rightarrow \infty$.

Thus we should study differently the following cases:

1. $E_{c} \rightarrow \infty$, but $L$ (and $d$ ) fixed. Since $\lim _{E_{c} \rightarrow \infty} \bar{s}_{0}=0$, the negative part of the Lyapunov spectrum collapses: $\lim _{E_{c} \rightarrow \infty} \chi_{i}^{-}=0$ for every $1 \leq i \leq N$. The hyperbolicity is lost, but the positive exponent $\chi_{0}^{+}=\log N$ survives. In particular, the entropy does not collapses.

2. $L \rightarrow \infty$, but $E_{c} \gg 1$ fixed. Here only a bounded piece $\chi_{1}^{-}, \ldots, \chi_{k}^{-}$of the Lyapunov spectrum collapses, $k=$ const. But the number of elements of this spectrum grows and in average $\left|\bar{\chi}^{-}\right| \rightarrow \infty$. In particular, $\left|\chi^{-}\right|_{\max } \rightarrow \infty$ as $L \rightarrow \infty$. Again, the positive exponent $\chi_{0}$ and entropy are preserved and though 
Boris Kruglikov, Martin Rypdal

we loose hyperbolicity there are many non-degenerate Oscelledec modes. Moreover they prevail over collapsing modes and so essentially the hyperbolicity is preserved as well.

3. Reparametrized model. This was introduced in 77.3 and require the renormalization: multiplication of waiting time by the function $\frac{\bar{\omega}}{\bar{\omega}+\bar{\tau}}$ along the trajectory. In this case the Lyapunov spectrum collapses to zero in any limit $E_{c} \rightarrow \infty$ and $L \rightarrow \infty$ and the hyperbolicity is completely lost.

Thus the exponential grow of the statistics is suppressed and we can observe power law statistic as is the basic idea of SOC-phenomenon. The corresponding SOC-exponents are related to the asymptotic of the Lyapunov spectrum (as discussed in $[\underline{\mathrm{BCK}})$, but are difficult to calculate analytically.

\section{Conclusion}

It is of importance to the paradigm of SOC to understand the mechanisms behind the behavior one observes numerically in the Zhang model and other sandpile models, and the aim of this paper is to provide a first step to a rigorous mathematical understanding of the dynamics of Zhang model.

Due to the singularities and non-invertibility of the model there existed very few applicable results, and hence we had to modify known results and develop some new methods in order to describe the dynamical properties of the model.

The result of this work is that the singularities play a modest role in the sense they do not change the main dynamical characteristics. However the effects of the singularities can be seen in the rich fractal structure of the spacial attractor.

Our analysis allows to take the thermodynamic limit of the main dynamical quantities, showing that the entropy vanishes and the Lyapunov-spectrum collapses after re-scaling the model. From a physical point of view this is interesting. Typically chaotic dynamics (positive entropy and positive Lyapunov exponents) is an indication of exponential speeds of mixing (short decay of correlations) $\mathrm{Ba}$, which is not compatible with the power-law statistics of the SOC-hypothesis. The loss of hyperbolicity in the thermodynamic limit hence supports the critical behavior observed numerically [J], Z, GD.

To conclude: We have shown that the Zhang model is a chaotic hyperbolic dynamical system, where all the entropy is produced by the random driving of the system and, due to singularities, the orbit structure is richer than for a topological Markov chain. The hyperbolicity indicates that under weak conditions there exists an SRB-measure (self-organization). In the thermodynamic limit the hyperbolicity is lost and we may expect power-law statistics (criticality). In practice the systems that are studied have finite size and finite critical energy. Hence they are chaotic, but with small entropy. The SOC-hypothesis is that these weakly chaotic systems have SRB-measures with the rates of convergence to these measures being exponential but slow compared to a unit step in an avalanche, causing the prevailing of power-law statistics. 
Dynamics and entropy of SOC

Finally note that modifications of the Zhang model are possible. For instance, different amounts of energy $\delta_{i}$ can be added to different sites $i \in \Lambda$ in the excitation process. This corresponds to the rectangular form of $\Lambda \subset \mathbb{Z}^{d}$ and uniform quantum of energy $\delta=\hbar$. We can also consider other spacial configurations $\Lambda$. In this way nearly Zhang models considered in Section 4.2 are natural. Another approach is to use a random amount of energy, i.e. stochastic $\delta: \Lambda \rightarrow \mathbb{R}_{+}$(this idea was used in the plasma physics $[\mathrm{KK}]$ ), which can also be presented as a skew product with piecewise affine fibers, but now over a solenoidal system. In all these theories the ideas from the present paper work well (though the thermodynamic limit will be sensitive to the form of $\Lambda$ ).

\section{A Topological entropy of piecewise affine maps}

\section{A.1. The Buzzi theorem and its generalization}

The statement as it is done in B1] does not apply to our situation. In B2] Buzzi noted that it extends to isometries and contractions. In fact, the assertion holds always, but since we cannot make a simple reference we write an adapted proof for the convenience of the reader.

Theorem 25. Let $X \subset \mathbb{R}^{d}$ be a bounded polytope and $f: X \rightarrow X$ a piecewise affine map. Then

$$
H_{\text {sing }}(f) \leq \lambda^{+}(f)+H_{\text {mult }}(f) \text {, }
$$

where

$$
\lambda^{+}(f)=\varlimsup_{n \rightarrow \infty} \sup _{x \in X} \frac{1}{n} \max _{k} \log \left\|\Lambda^{k} d_{x} f^{n}\right\| .
$$

Proof. Let $\mathcal{P}=\left\{P_{i}\right\}$ be the continuity partition and $f_{i}:=\left.f\right|_{P_{i}}$ be affine maps. Fix $\epsilon>0$ and $T=T(\epsilon) \geq \frac{d}{\epsilon} \log (\sqrt{d}+1)$ such that for $n \geq T$ we have:

$$
\operatorname{mult}\left(\mathcal{P}^{n}\right) \leq \exp \left(\left(H_{\text {mult }}(f)+\epsilon\right) n\right),\left\|\Lambda d f^{n}\right\| \leq \exp \left(\left(\lambda^{+}(f)+\epsilon\right) n\right) .
$$

Take $r=r(\epsilon)$ to be compatible with the partition $\mathcal{P}^{T}$ (for $f^{T}$ ), i.e. any $r$-ball intersects maximally mult $\left(\mathcal{P}^{T}\right)$ partition elements.

We will prove that each non-empty cylinder $C(\mathbf{a})=\left[P_{a_{0}} \ldots P_{a_{l T-1}}\right]$ of length $|\mathbf{a}|=l T$ can be partitioned into a collection $Q(\mathbf{a})=\{W\}$ satisfying the following properties:

1. $\sum_{|\mathbf{a}|=l T} \operatorname{card}(Q(\mathbf{a})) \leq C_{0} \exp \left(\left(\lambda^{+}(f)+H_{\text {mult }}(f)+3 \epsilon\right) l T\right)$

2. $\operatorname{diam}\left(f_{a_{l T-1}} \circ \cdots \circ f_{a_{0}}(W)\right) \leq r$.

Let us prove this claim by induction assuming it holds for some $l \geq 0$. The base of induction is obvious and $C_{0}$ is the minimal cardinality of an $r / 2$-ball cover.

Take a partition element $W \in Q(\mathbf{a})$ that is used to cover the cylinder $\left[P_{a_{0}} \ldots P_{a_{l T-1}}\right]$. By the induction hypothesis it has diameter less than $r$, so 
it can be continued to cover a non-empty cylinder of length $(l+1) T$ in at most $\operatorname{mult}\left(\mathcal{P}^{T}\right)$ ways. So to cover the cylinders $\left[P_{a_{0}} \ldots P_{a_{l T-1}} P_{b_{0}} \ldots P_{b_{T-1}}\right]$ we make a division of $W$ :

$$
W=\bigcup_{i=1}^{\gamma} W_{i}^{\prime}, \gamma \leq \operatorname{mult}\left(\mathcal{P}^{T}\right) .
$$

Let

$$
W_{i}^{\prime \prime}=f_{a_{T l-1}} \circ \cdots \circ f_{a_{0}}\left(W_{i}^{\prime}\right)
$$

and

$$
W_{i}^{\prime \prime \prime}=f_{b_{T-1}} \circ \cdots \circ f_{b_{0}}\left(W_{i}^{\prime \prime}\right) .
$$

By the assumption $\operatorname{diam}\left(W_{i}^{\prime \prime}\right)<r$ for all $i=1, \ldots, \gamma$, but the sets $W_{i}^{\prime \prime \prime}$ may have greater diameter. We need to divide the sets $W_{i}^{\prime \prime \prime}$ so that they have diameter less than $r$, and then pull this refinement back to the partition of sets $W_{i}^{\prime}$.

Let $L$ be the differential of $f_{b_{T-1}} \circ \cdots \circ f_{b_{0}}$ on $W_{i}^{\prime \prime}$. We can assume that $L$ is symmetric and take $\left\{e_{k}\right\}$ to be a basis of eigenvectors corresponding to eigenvalues $\lambda_{1}, \ldots, \lambda_{d}$. Let $\left\{v_{k}\right\}$ be a basis in the vector subspace corresponding to $W_{i}^{\prime \prime \prime}$. We can choose this basis to be orthonormal and triangular with respect to $\left\{e_{k}\right\}$. Divide $W_{i}^{\prime \prime \prime}$ by the hyperplanes

$$
\psi_{j}(x) \stackrel{\text { def }}{=}\left\langle v_{j}, x\right\rangle=p \frac{r}{\sqrt{d}}, \quad p \in \mathbb{Z}, \quad j=1, \ldots, d .
$$

This defines cells $\tilde{W}$ of diameter less than $r$. Since $\psi_{j}\left(W_{i}^{\prime \prime \prime}\right)=\psi_{j}\left(L\left(W_{i}^{\prime \prime}\right)\right)$ has diam $\leq\left|\lambda_{i}\right| r$, the number of cells $\tilde{W}$ needed to cover $W_{i}^{\prime \prime \prime}$ is less than or equal to

$$
(\sqrt{d}+1)^{d}\left|\lambda_{1}\right|^{+} \ldots\left|\lambda_{d}\right|^{+} \leq(\sqrt{d}+1)^{d}\left\|\Lambda d f^{n}\right\| \leq \exp \left(\left(\lambda^{+}(f)+2 \epsilon\right) T\right),
$$

where $\left|\lambda_{i}\right|^{+}=\max \left\{\left|\lambda_{i}\right|, 1\right\}$.

Therefore the total cardinality of the new partition is less than or equal to

$$
\begin{array}{r}
\operatorname{mult}\left(\mathcal{P}^{T}\right) \exp \left(\left(\lambda^{+}(f)+2 \epsilon\right) T\right) \exp \left(\left(\lambda^{+}(f)+H_{\text {mult }}(f)+3 \epsilon\right) l T\right) \\
\leq \exp \left(\left(\lambda^{+}(f)+H_{\text {mult }}(f)+3 \epsilon\right)(l+1) T\right) .
\end{array}
$$

This proves the statement.

The theorem holds as well for most degenerate piece-wise affine systems, but there can be problems with $H_{\text {mult }}(f)$. Namely the latter is not defined if the image of continuity domain contains a boundary face of a continuity domain. But if we assume the image and the faces always meet transversally, no problems occur and the above theorem applies literally.

In degenerate cases of the Zhang model, the above requirement holds for most parameters. For instance, if $N=2$ and $\epsilon=0$, then all $E_{c} \notin \mathbb{Z}$ satisfy the request. For integer $E_{c}$ the theory fails, but one can look just to the whole image set, which has dimension $<N$ : its Poincaré return map is piece-wise affine and it satisfies the requirements. In the above example $N=2, \epsilon=0$ the dynamics is confined to two one dimensional lines, whence the multiplicity entropy is zero (by dimensional reasons) and $h_{\mathrm{top}}(F)=\log 2$ in this case. 
Dynamics and entropy of SOC

\section{A.2. Entropy of conformal piecewise affine skew-products}

We say that an affine map is conformal modulo degenerations if the image on some subspace transversal to the kernel is mapped conformally to its image. A piecewise affine map is said to be conformal modulo degenerations if all its affine components are conformal modulo degenerations. We will assume that degenerations satisfy the transversality requirement of A.1

In [KR1] we noticed that $H_{\text {mult }}=0$ for piece-wise affine conformal maps. This easily extends to allow degenerations. Now we consider a more general situation of skew-product systems of Zhang's type.

Theorem 26. Let $f_{i}: X \rightarrow X$ be piece-wise affine non-strictly contracting and conformal modulo degenerations, $i=0, \ldots, N-1$. Define $F: \Sigma_{N}^{+} \times X \rightarrow \Sigma_{N}^{+} \times X$ by the formula $F(\mathbf{t}, x)=\left(\sigma_{N}^{+}(\mathbf{t}), f_{t_{0}}(x)\right), \mathbf{t}=t_{0} t_{1} \cdots \in \Sigma_{N}^{+}, x \in X \subset \mathbb{R}^{d}$. Then we have: $h_{\mathrm{top}}(F)=\log N$ (so that a-posteriori the variational principle holds).

Remark 11. For $N=2$ and $\epsilon=0$ the affine components have rank 1, and hence Theorem [26] shows $h_{\mathrm{top}}(F)=\log N$. The same holds for $E_{c}=\epsilon=1 / 3$.

Proof. It follows from Theorem 25 that it suffices to prove that $H_{\text {mult }}(F)=0$.

To achieve the desired equality note that preimages of the time-like singularity planes $\{t=n / N\}$ never intersect under inverse iterations of $F(t, x)=$ $\left(N t \bmod 1, f_{[N t]}(x)\right)$. Thus $H_{\text {mult }}(F)=\sup _{\mathbf{t}} H_{\text {mult }}\left(f_{\mathbf{t}}\right)$. We will prove that $H_{\text {mult }}\left(f_{\mathbf{t}}\right)=0$ for all $\mathbf{t} \in \Sigma_{N}^{+}$.

A piecewise affine map can be considered as an ordered triple $(X, \mathcal{P}, f)$, where $X$ is a polytope in $\mathbb{R}^{d}, \mathcal{P}=\left\{P_{i}\right\}$ is a partition of $X$ made up of pairwise disjoined polytopes (with certain faces of boundary included, so that the whole boundary is distributed between polytopes) and $f_{i}:=\left.f\right|_{P_{i}}: P_{i} \rightarrow X$ are affine maps. We let $X^{\prime}=\cup \operatorname{Int}\left(P_{i}\right)$ and $\operatorname{Sing}(f)=X \backslash X^{\prime}$.

For a piecewise affine map $(X, \mathcal{P}, f)$ and a point $x \in X$ construct a piecewise affine map $\left(X_{x}, \mathcal{P}_{x}, f_{x}\right)$, called the differential of $f$ at $x$, by letting

1. $X_{x}=\left\{y \in \mathbb{R}^{d} \mid \exists \epsilon_{0}>0\right.$ s.t. $\left.\forall \epsilon \in\left(0, \epsilon_{0}\right): x+\epsilon y \in X\right\} \subset \mathbb{R}^{d}$ is the tangent cone to $X$.

2. $\mathcal{P}_{x}$ is the partition of $X_{x}$ consisting of non-empty sets

$$
P_{x}=\left\{y \in \mathbb{R}^{d} \mid \exists \epsilon_{0}>0 \text { s.t. } \forall \epsilon \in\left(0, \epsilon_{0}\right): x+\epsilon y \in P\right\},
$$

where $P \in \mathcal{P}$.

3. $f_{x}: P_{x} \rightarrow X_{f(x)}$ is the collection of maps

$$
f_{x}(y)=\lim _{\epsilon \rightarrow 0^{+}} \frac{f(x+\epsilon y)-\lim _{\delta \rightarrow 0^{+}} f(x+\delta y)}{\epsilon}, \quad P_{x} \in \mathcal{P}_{x} .
$$

Consider iterated differentials and denote $f_{x_{1}, \ldots, x_{n}}:=\left(\ldots\left(f_{x_{1}}\right)_{x_{2}} \ldots\right)_{x_{n}}$. Note that $\left(f_{\mathbf{t}}^{n}\right)_{x}=\left(f_{t_{n-1}}\right)_{f_{\mathbf{t}}^{n}(x)} \circ \ldots\left(f_{t_{1}}\right)_{f_{\mathbf{t}}^{1}(x)} \circ\left(f_{t_{0}}\right)_{x}$. 
Boris Kruglikov, Martin Rypdal

Proposition 27. There exists a constant $C \in \mathbb{R}_{+}$such that for any subspace $W \subset \mathbb{R}^{d}$ and any $\left(x_{1}, \ldots, x_{r}\right) \in X \times W^{r-1}$ we have:

$$
\operatorname{mult}\left(\left(\left.f_{\mathbf{t}}^{n}\right|_{W}\right)_{x_{1}, \ldots, x_{r}}\right) \leq C\left(\sup _{V \subset \mathbb{R}^{d}} \sup _{y_{1}, \ldots, y_{r+1}} \operatorname{mult}\left(\left.f_{\mathbf{t}}\right|_{V}\right)_{y_{1}, \ldots, y_{r+1}}\right)^{n}, \quad \forall n \geq 0,
$$

where the collection of points $\left(y_{1}, \ldots, y_{r+1}\right)$ runs over $X \times V^{r}$ with the condition $\operatorname{rank}\left(y_{2}, \ldots, y_{r+1}\right)+\operatorname{codim} V=\operatorname{rank}\left(x_{2}, \ldots, x_{r}\right)+\operatorname{codim} W+1$.

The theorem follows from this, because for

$$
\begin{gathered}
\mu(r)=\varlimsup_{n \rightarrow \infty} \frac{1}{n} \log _{\substack{y_{1} \in X, y_{2} \ldots, y_{r} \in V \subset \mathbb{R}^{d} \\
\operatorname{rank}\left(y_{2}, \ldots, y_{r}\right)+\operatorname{codim} V=r-1}} \operatorname{sult}\left(\left.f_{\mathbf{t}}^{n}\right|_{V}\right)_{y_{1}, \ldots, y_{r}} \\
\end{gathered}
$$

we have: $H_{\text {sing }}\left(f_{\mathbf{t}}\right)=\mu(1) \leq \mu(2) \leq \cdots \leq \mu(d+1)=0$.

To prove the proposition note that when $r=0$ we have (in this case we do not need $W, V)$ :

$$
\operatorname{mult}\left(f_{\mathbf{t}}^{n}\right) \leq\left(\sup _{x \in X} \operatorname{mult}\left(f_{\mathbf{t}}\right)_{x}\right)^{n}=\left(\max _{0 \leq j<N} \sup _{x \in X} \operatorname{mult}\left(f_{j}\right)_{x}\right)^{n} .
$$

Let $r \geq 1$. Consider the continuity partition $\mathcal{P}_{x_{1}, \ldots, x_{r}}^{\mathbf{t}}$ for $\left(f_{\mathbf{t}}\right)_{x_{1}, \ldots, x_{r}}$, which is just the continuity partition $\mathcal{P}_{x_{1}, \ldots, x_{r}}^{\left(t_{0}\right)}$ of $\left(f_{t_{0}}\right)_{x_{1}, \ldots, x_{r}}$ (one iteration), and let $\mathcal{P}_{x_{1}, \ldots, x_{r}}^{n, \mathbf{t}}$ for $\left(f_{\mathbf{t}}^{n}\right)_{x_{1}, \ldots, x_{r}}$ be the iterated partition. Note that the latter is the collection of all non-empty intersections

$P^{t_{0}} \cap\left(f_{t_{0}}\right)_{P^{t_{0}}}^{-1}\left(P^{t_{1}}\right) \cap\left(f_{t_{0}}\right)_{P^{t_{0}}}^{-1}\left(f_{t_{1}}\right)_{P^{t_{1}}}^{-1}\left(P^{t_{2}}\right) \cap \cdots \cap\left(f_{t_{0}}\right)_{P^{t_{0}}}^{-1} \ldots\left(f_{t_{n-1}}\right)_{P^{t_{n-1}}}^{-1}\left(P^{t_{n}}\right)$,

where $P^{t_{i}}$ are elements of $\mathcal{P}_{x_{1}, \ldots, x_{r}}^{\left(t_{i}\right)}$ and $f_{P}$ denotes the restriction of the (differential of the) map to the corresponding continuity domain. Every element of these partitions is invariant under the shift by vectors from $\operatorname{span}\left(x_{2}, \ldots, x_{r}\right)$. Therefore it intersects the unit sphere $S_{1}\left(x_{2}, \ldots, x_{r}\right)^{\perp}$ in the orthogonal complement. Consider the induced partition on the sphere and refine it so that every element has diameter no greater than $\varepsilon$. Denote by $n\left(\mathcal{P}_{x_{1}, \ldots, x_{r}}^{n, \mathbf{t}}, \varepsilon\right)$ the minimal cardinality of such a refinement. Let also $m\left(\mathcal{P}_{x_{1}, \ldots, x_{r}}^{\left(t_{i}\right)}, \varepsilon\right)$ be the maximal number of elements of $\mathcal{P}_{x_{1}, \ldots, x_{r}}^{\left(t_{i}\right)}$ that an $\varepsilon$-ball $B(y, \varepsilon) \cap S_{1}^{\perp}$ of $S_{1}\left(x_{2}, \ldots, x_{r}\right)^{\perp}$ can meet.

Denote by $n(\mathcal{P} \cap W, \varepsilon), m(\mathcal{P} \cap W, \varepsilon)$ the corresponding quantities in the subspace $W$. Then from the above formula for the iterated partition:

$$
n\left(\mathcal{P}_{x_{1}, \ldots, x_{r}}^{n+1, \mathbf{t}} \cap W, \varepsilon\right) \leq n\left(\mathcal{P}_{x_{1}, \ldots, x_{r}}^{n, \mathbf{t}} \cap W, \varepsilon\right) \cdot m\left(\mathcal{P}_{y_{1}, \ldots, y_{r}}^{\left(t_{n+1}\right)} \cap V, \varepsilon\right),
$$

where $y_{1}=f_{\mathbf{t}}^{n}\left(x_{1}\right), y_{2}=f_{\mathbf{t}}^{n \prime}\left(x_{2}\right), \ldots, y_{r}=f_{\mathbf{t}}^{n \prime}\left(x_{r}\right)$ and $V=f_{\mathbf{t}}^{n \prime}(W)$ with $f_{\mathbf{t}}^{n \prime}=\left(f_{\mathbf{t}}^{n}\right)_{x_{1}, \ldots, x_{r}}$. Therefore

$$
n\left(\mathcal{P}_{x_{1}, \ldots, x_{r}}^{n+1, \mathbf{t}} \cap W, \varepsilon\right) \leq n\left(\mathcal{P}_{x_{1}, \ldots, x_{r}}^{n, \mathbf{t}} \cap W, \varepsilon\right) \cdot \sup _{V} \sup _{y_{1}, \ldots, y_{r}} m\left(\mathcal{P}_{y_{1}, \ldots, y_{r}}^{\left(t_{n+1}\right)} \cap V, \varepsilon\right),
$$


where the supremum is taken over all $V \subset \mathbb{R}^{d}$ and $\left(y_{1}, \ldots, y_{r}\right) \in X \times V^{r-1}$ such that codimension of $\left\langle y_{2} \ldots, y_{r}\right\rangle$ in $V$ equals codimension of $\left\langle x_{2} \ldots, x_{r}\right\rangle$ in $W$.

Since for a fixed $\varepsilon$ the number $n\left(\mathcal{P}_{x_{1}, \ldots, x_{r}}^{t_{0}}, \varepsilon\right)$ is finite and

$$
m\left(\mathcal{P}_{y_{1}, \ldots, y_{r}}^{\left(t_{i}\right)} \cap V, \varepsilon\right) \leq \sup _{y \in S_{1}\left(y_{1}, \ldots, y_{r}\right)^{\perp} \cap V}\left|\mathcal{P}_{y_{1}, \ldots, y_{r}}^{\left(t_{i}\right)} \cap B(y, \varepsilon) \cap V\right|,
$$

the claim follows from the following statement. Fix $i \in[0, N)$.

Lemma 28. There exists $\varepsilon>0$ (depending only on i) such that for all $V \subset$ $\mathbb{R}^{d}$ and all $\left(y_{1}, \ldots, y_{r}\right) \in X \times V^{r-1}$, with $\operatorname{rank}\left(y_{2}, \ldots, y_{r}\right)<\operatorname{dim} V$, and $y \in$ $S_{1}\left(y_{2}, \ldots, y_{r}\right)^{\perp} \cap V$ there exists $y^{\prime} \in\left(y_{2}, \ldots, y_{r}\right)^{\perp} \cap V$ satisfying:

$$
\left|\mathcal{P}_{y_{1}, \ldots, y_{r}}^{(i)} \cap B(y, \varepsilon) \cap V\right| \leq \operatorname{mult}\left(\left(\left.f_{i}\right|_{V}\right)_{y_{1}, \ldots, y_{r}, y^{\prime}}\right) .
$$

This statement, modulo our notations and restrictions to $V$, is proved in [B2]. The proposition and hence the theorem follow.

\section{A.3. Estimates on entropy by angular expansion rates}

It is possible to estimate the effect of angular expansion on the topological entropy of a piecewise affine map $f: X \rightarrow X$ by its spherizations. Define the piecewise smooth map $d_{x}^{(s)} f: S T_{x} X \rightarrow S T_{f(x)} X$ given at $x \in X^{\prime}$ by the formula

$$
d_{x}^{(s)} f(v)=\frac{d_{x} f(v)}{\left\|d_{x} f(v)\right\|} .
$$

For $x \in \operatorname{Sing}(f)$ and $v \notin T_{x} \operatorname{Sing}(f)$ (the tangent cone) we let $d_{x}^{(s)} f(v)=$ $\lim _{\epsilon \rightarrow+0} d_{x+\epsilon v}^{(s)} f(v)$. For other $(x, v) \in S T X$ the map is not defined. The angular expansion of $f$ is exactly the expansion in the fibers of its spherization.

If $d_{x} f$ is degenerate we restrict to the orthogonal component of its kernel, and consider the map

$$
\left.d_{x} f\right|_{\operatorname{Ker}\left(d_{x} f\right)^{\perp}}: \operatorname{Ker}\left(d_{x} f\right)^{\perp} \rightarrow \operatorname{Im}\left(d_{x} f\right) .
$$

Then the map $S_{x}(f)=\left.d_{x}^{(s)} f\right|_{\operatorname{Ker}\left(d_{x} f\right)^{\perp}}: S \operatorname{Ker}\left(d_{x} f\right)^{\perp} \rightarrow S \operatorname{Im}\left(d_{x} f\right)$ between $\left(\operatorname{rank}\left(d_{x} f\right)-1\right)$-dimensional spheres is given by the formula

$$
v \mapsto \frac{\left.d_{x} f\right|_{\operatorname{Ker}\left(d_{x} f\right)^{\perp}}(v)}{\left\|\left.d_{x} f\right|_{\operatorname{Ker}\left(d_{x} f\right)^{\perp}}(v)\right\|} .
$$

For $i<d$ we define

$$
\rho_{i}(f)=\varlimsup_{n \rightarrow \infty} \frac{1}{n} \sup _{(x, v)} \max _{0 \leq k \leq i} \log \left\|\Lambda^{k} d_{v} S_{x}\left(f^{n}\right)\right\| .
$$

Let $m_{*}=\min _{x} \operatorname{dim} \operatorname{Ker}\left(d_{x} f\right)$ and $d_{*}=d-m_{*}=\max _{x} \operatorname{rank}\left(d_{x} f\right)$, where $d$ is the dimension of $X$. The numbers $\rho_{i}(f)$ can be non-zero only for $i<d_{*}$. 


\section{Boris Kruglikov, Martin Rypdal}

We have: $\rho_{0}(f)=0$. The number $\rho_{1}(f)$ measures the maximal exponential rate with which angles can increase under the map $f$. The numbers $\rho_{i}(f)$ for $i<d$ measure the maximal rate of expansion of the restrictions to $i$-dimensional spheres. If $f$ is conformal, then $\rho_{i}(f)=0$ for all $i$.

Theorem 29 ([KR2]). For piece-wise affine maps $H_{\text {mult }}(f) \leq \sum_{i=1}^{d_{*}-1} \rho_{i}(f)$.

We define the maximal expansion rate

$$
\lambda_{\max }(f)=\varlimsup_{n \rightarrow \infty} \sup _{x} \frac{1}{n} \log \left\|d_{x} f^{n}\right\|,
$$

and the minimal finite expansion rate

$$
\lambda_{\min }(f)=-\varlimsup_{n \rightarrow \infty} \sup _{x} \frac{1}{n} \log \left\|\left(\left.d_{x} f^{n}\right|_{\operatorname{Ker}\left(d_{x} f^{n}\right)^{\perp}}\right)^{-1}\right\| .
$$

In [KR2] we show that

$$
\rho_{i}(f) \leq i\left(\lambda_{\max }(f)-\lambda_{\min }(f)\right) .
$$

This gives the following result (the same bound holds for $h_{\text {top }}(f)$ ):

Theorem 30. For a piecewise affine map $f$ it holds:

$$
H_{\text {sing }}(f) \leq \lambda^{+}(f)+\frac{d_{*}\left(d_{*}-1\right)}{2}\left(\lambda_{\max }(f)-\lambda_{\min }(f)\right) .
$$

\section{B. Generalization of the Moran formula}

Consider an IFS $\left(M^{d}, f_{1}, \ldots, f_{N}\right)$ on a Riemannian manifold $M$, where the maps $f_{i}$ can possess singularities, but we assume that they are mild in a sense that the number of continuity domains is finite, any of them has piece-wise smooth boundary and the map, restricted to any of the domains, smoothly extends to the adjacent singularities (this is the case of the Zhang model).

Remark that the IFS can be interpreted as the dynamical system $\left(\Sigma_{N}^{+} \times\right.$ $M, F), \hat{f}(\mathbf{t}, x)=\left(\sigma_{N}^{+} \mathbf{t}, f_{t_{0}} x\right)$. Attractor $\mathcal{Y}$ of the IFS can be defined via the attractor of the extended system $F$, which has the form $\mathcal{A}=\Sigma_{N}^{+} \times \mathcal{Y}$.

Let $\|\cdot\|$ be the norm on $T M$ generated by the metric on $M$. Denote

$$
s_{i}^{+}=\max _{x \in M}\left\|d_{x} f_{i}\right\|, \quad s_{i}^{-}=\left(\max _{x \in M}\left\|d_{x} f_{i}^{-1}\right\|\right)^{-1} .
$$

We assume that the maps are non-degenerate (this is just for simplicity of arguments) and strictly contracting, so that $0<s_{i}^{-} \leq s_{i}^{+}<1$.

Let $\eta=\max _{x \in M} \#\left\{i \mid x=f_{i}\left(y_{i}\right)\right.$ for some $\left.y_{i} \in \mathcal{Y}\right\}$ be the maximal multiplicity of overlaps and $\varkappa_{i}=\max _{x \in \mathcal{Y}} \#\left\{y \in \mathcal{Y} \mid x=f_{i}(y)\right\}$ be the maximal multiplicity of self-overlaps (we assume it is finite) on the attractor. Denote also by $\vartheta_{i}$ the multiplicity of the continuity partition for $f_{i} \mid \mathcal{y}$, i.e. the maximal number of continuity domains intersecting the attractor and meeting at one point of it. 
Theorem 31. Let $\underline{D}=\alpha, \bar{D}=\beta$ be the solutions of the equations

$$
\sum_{i=1}^{N} \frac{1}{\varkappa_{i}}\left|s_{i}^{-}\right|^{\alpha}=\eta, \quad \sum_{i=1}^{N} \vartheta_{i}\left|s_{i}^{+}\right|^{\beta}=1 .
$$

Then the Hausdorff dimension of the attractor satisfies:

$$
\underline{D} \leq \operatorname{dim}_{H}(\mathcal{Y}) \leq \bar{D} .
$$

In addition to Hausdorff dimension we will need some other dimensional characteristics (see [P2 for details). Denote by $\mathfrak{N}(X, \delta)$ the minimal cardinality of covers of $X$ by balls of radius $\delta$. Then the lower and upper box dimensions are defined by the formula:

$$
\underline{\operatorname{dim}}_{B}(X)=\varliminf_{\delta \rightarrow+0} \log \mathfrak{N}(X, \delta) / \log \frac{1}{\delta}, \quad \overline{\operatorname{dim}}_{B}(X)=\varlimsup_{\delta \rightarrow+0} \log \mathfrak{N}(X, \delta) / \log \frac{1}{\delta} .
$$

When these quantities are equal, their value is also called fractal dimension.

Consider a Borel probability measure $\mu \in \mathcal{M}(X)$ (an SRB-measure on the attractor can be taken in the SOC-context if it exists). The upper and lower pointwise dimensions are defined then as

$$
\underline{d}_{\mu}(x)=\varliminf_{\delta \rightarrow+0} \log \mu(B(x, \delta)) / \log \delta, \quad \bar{d}_{\mu}(x)=\varlimsup_{\delta \rightarrow+0} \log \mu(B(x, \delta)) / \log \delta .
$$

When they are equal and constant a.e. the measure $\mu$ is called exact-dimensional. This is precisely the case, when $\operatorname{supp} \mu=X$ and we have equality in the general chain of inequalities (together with (13) below):

$$
\text { ess. inf } \underline{d}_{\mu}(x) \leq \operatorname{dim}_{\mathrm{H}}(X) \leq \underline{\operatorname{dim}}_{B}(X) \leq \overline{\operatorname{dim}}_{B}(X),
$$

where by essential infimum we mean its upper bound taken over all subsets $U \subset X$ of measure 1 (and similar for ess. $\sup \bar{d}_{\mu}(x)$ ). The last two inequalities are known and the first one follows from the inequality ess. inf $\underline{d}_{\mu}(x) \leq \operatorname{dim}_{\mathrm{H}}(\mu)$ ([P2] $)$, where $\operatorname{dim}_{H}(\mu)=\lim _{\delta \rightarrow+0} \inf \left\{\operatorname{dim}_{H}(Z) \mid \mu(Z)>1-\delta\right\}$.

Other dimensional characteristics of the measure are defined similarly and satisfy:

$$
\underline{\operatorname{dim}}_{B}(\mu) \leq \overline{\operatorname{dim}}_{B}(\mu) \leq \operatorname{ess} \cdot \sup \bar{d}_{\mu}(x)
$$

Note that $\overline{\operatorname{dim}}_{B}(\mu) \leq \overline{\operatorname{dim}}_{B}(X)$, while the quantities ess. $\sup \bar{d}_{\mu}(x)$ and $\overline{\operatorname{dim}}_{B}(X)$ are in general incomparable.

The known formulas for the Hausdorff and other dimensions are generalizations of Moran's result ( $\underline{\mathrm{M}}, \underline{\mathrm{H}})$ ) and are based on the Bowen's equation (using the idea of coding); in this case one usually obtains exact-dimensionality [P2]. In the SOC-context coding becomes problematic in the presence of singularities (unless the properties of the SRB-measure are clarified) and thus we cannot easily establish exact-dimensionality or formula for the dimension.

We prove instead the inequality of the theorem for all the various dimensions from (12) and (13), which we denote just by $\operatorname{dim}(\mathcal{Y})$ :

$$
\underline{D} \leq \operatorname{dim}(\mathcal{Y}) \leq \bar{D} .
$$


Proof. Let us consider at first the upper box dimension $\overline{\operatorname{dim}}_{B}(\mathcal{Y})$. The function $\mathfrak{N}$ satisfies the inequalities:

$$
\frac{1}{\varkappa_{i}} \mathfrak{N}\left(\mathcal{Y}, \delta / s_{i}^{-}\right) \leq \mathfrak{N}\left(f_{i}(\mathcal{Y}), \delta\right) \leq \vartheta_{i} \mathfrak{N}\left(\mathcal{Y}, \delta / s_{i}^{+}\right)
$$

The inequality from above is obtained as follows. Let $\mathcal{S}=\left\{x_{j}\right\}$ be a $\delta$-spanning set, i.e. a collection of points from $X$ with $U_{\delta}(\mathcal{S})=X$. Then $f_{i}(\mathcal{S})=\left\{f_{i}\left(x_{j}\right)\right\}$ may fail to be a $\delta \cdot s_{i}^{+}$-spanning set thanks to singularities. Whenever $\delta \ll 1$, every $\delta$-ball intersects maximally $\vartheta_{i}$ domains of continuity for $f_{i}$ meeting $\mathcal{Y}$. Then we need to add maximally $\vartheta_{i}$ points for each ball $U_{\delta}\left(x_{j}\right)$ intersecting singularities. The inequality from below is proved similarly.

Now we have:

$$
\mathcal{Y}=f_{1}(\mathcal{Y}) \cup \cdots \cup f_{N}(\mathcal{Y})
$$

and the same for $U_{\delta}$-neighborhoods. This implies:

$$
\mathfrak{N}(\mathcal{Y}, \delta) \leq \sum_{i=1}^{N} \mathfrak{N}\left(f_{i}(\mathcal{Y}), \delta\right) \leq \sum_{i=1}^{N} \vartheta_{i} \mathfrak{N}\left(\mathcal{Y}, \delta / s_{i}^{+}\right)
$$

Denote $\sigma(\delta)=\mathfrak{N}(\mathcal{Y}, \delta) \delta^{\overline{\operatorname{dim}}_{B}(\mathcal{Y})}$. This functions grows sub-polynomially:

$$
\varlimsup_{\delta \rightarrow+0} \frac{\log \sigma(\delta)}{\log 1 / \delta}=0
$$

Lemma 32. Let $\lambda_{i}>1$ be some numbers and $p_{i}>0$ be some probabilities, $\sum_{i=1}^{N} p_{i}=1$. Then (15) implies:

$$
\varliminf_{\delta \rightarrow+0} \frac{\sum_{i=1}^{N} p_{i} \sigma\left(\lambda_{i} \delta\right)}{\sigma(\delta)} \leq 1 .
$$

Proof. Suppose the lower limit is $>\kappa>1$. Then for every sufficiently small $\delta$ there exists $i \in[1, N]$ such that $\sigma\left(\lambda_{i} \delta\right) \geq \kappa \sigma(\delta)$.

Denote $\bar{\lambda}=\max _{1 \leq i \leq N} \lambda_{i}$. Let $C=\max _{\delta \in[1 / \bar{\lambda}, 1]} \sigma(\delta)$. Then:

$$
\sigma(\delta) \leq \frac{1}{\kappa} \sigma\left(\lambda_{i_{1}} \delta\right) \leq \frac{1}{\kappa^{2}} \sigma\left(\lambda_{i_{1}} \lambda_{i_{2}} \delta\right) \leq \cdots \leq \frac{1}{\kappa^{s(\delta)}} \sigma\left(\lambda_{i_{1}} \ldots \lambda_{i_{s(\delta)}} \delta\right),
$$

where $s(\delta)$ is the first number such that $\lambda_{i_{1}} \ldots \lambda_{i_{s(\delta)}} \delta \in[1 / \bar{\lambda}, 1]$. This number can be estimated as follows: $s(\delta) \geq-\log \delta / \log \bar{\lambda}-1$, whence:

$$
\frac{\log \sigma(\delta)}{\log 1 / \delta} \leq \frac{\log C-s(\delta) \log \kappa}{\log 1 / \delta} \leq \frac{\log (C \kappa)}{\log 1 / \delta}-\frac{\log \kappa}{\log \bar{\lambda}}
$$

Therefore $\overline{\lim }_{\delta \rightarrow+0} \frac{\log \sigma(\delta)}{\log 1 / \delta} \leq-\frac{\log \kappa}{\log \lambda}<0$ and we get a contradiction. This proves the lemma. 
Now to obtain the inequality from above for $\overline{\operatorname{dim}}_{B}(\mathcal{Y})$ divide (14) by $\mathfrak{N}(\mathcal{Y}, \delta)$. Denoting $\varpi=\sum_{i=1}^{N} \vartheta_{i}\left|s_{i}^{+}\right|^{\overline{\operatorname{dim}}_{B}(\mathcal{Y})}, \lambda_{i}=1 / s_{i}^{+}$and $p_{i}=\vartheta_{i}\left|s_{i}^{+}\right|^{\overline{\operatorname{dim}}_{B}(\mathcal{Y})} / \varpi$ we get:

$$
\frac{1}{\varpi} \leq \sum_{i=1}^{N} p_{i} \frac{\sigma\left(\lambda_{i} \delta\right)}{\sigma(\delta)} .
$$

Thus Lemma 32 implies that $1 / \varpi \leq 1$ or

$$
1 \leq \sum_{i=1}^{N} \vartheta_{i}\left|s_{i}^{+}\right|^{\operatorname{dim} \mathcal{Y}}
$$

and the first claim $\overline{\operatorname{dim}}_{B}(\mathcal{Y}) \leq \bar{D}$ follows from the contraction $\left|s_{i}^{+}\right|<1$. The same arguments show another statement that $\bar{d}_{\mu}(x) \leq \bar{D}$.

The inequality from below follows from

$$
\mathfrak{N}(\mathcal{Y}, \delta) \geq \frac{1}{\eta} \sum_{i=1}^{N} \mathfrak{N}\left(f_{i}(\mathcal{Y}), \delta\right) \geq \frac{1}{\eta} \sum_{i=1}^{N} \frac{1}{\varkappa_{i}} \mathfrak{N}\left(\mathcal{Y}, \delta / s_{i}^{-}\right),
$$

which implies $\sum_{i=1}^{N} \frac{1}{\varkappa_{i}}\left|s_{i}^{-}\right| \underline{\operatorname{dim}}_{B}(\mathcal{Y}) \leq \eta$ and the same for the lower pointwise dimension: $\underline{d}_{\mu}(x) \geq \underline{D}$ a.e.

In this case we should define

$$
\sigma(\delta)=\mathfrak{N}(\mathcal{Y}, \delta) \delta \underline{\operatorname{dim}}_{B}(\mathcal{Y}) \text { or } \sigma(\delta)=(\text { ess. inf }-\log \mu(B(x, \delta))) \delta \underline{\operatorname{dim}}_{B}(\mu)
$$

respectively and use

Lemma 33. Let $\lambda_{i}>1$ be some numbers and $p_{i}>0$ be some probabilities, $\sum_{i=1}^{N} p_{i}=1$. Then:

$$
\varliminf_{\delta \rightarrow+0} \frac{\log \sigma(\delta)}{\log 1 / \delta}=0 \Longrightarrow \varlimsup_{\delta \rightarrow+0} \frac{\sum_{i=1}^{N} p_{i} \sigma\left(\lambda_{i} \delta\right)}{\sigma(\delta)} \geq 1 .
$$

This is proved similarly to Lemma 32 The inequalities for the Hausdorff dimension follows now from (12).

Acknowledgements. We thank J. Schmeling and Y. Pesin for several stimulating discussions and references. M. Rypdal thanks E. Mjølhus for some useful questions and comments. We are grateful to the organizers of the Clay Mathematics Institute/MSRI Workshop on Recent Progress in Dynamics (2004), where we finished the final stage of the paper.

\section{References}

[AR] L. Abramov, V. Rokhlin, The entropy of a skew product of measure-preserving transformations, Amer. Math. Soc. Transl. Ser. 2, 48 (1966), 255-265. 


\section{Boris Kruglikov, Martin Rypdal}

[Ba] V. Baladi, Spectrum and Statistical Properties of Chaotic Dynamics, Proc. 3rd European Congress of Mathematics, Birkhauser (2001), 203-224.

[B1] J. Buzzi, Intrinsic ergodicity of affine maps in $[0,1]^{d}$, Mh. Math. 124 (1997), 97-118.

[B2] J. Buzzi, Piecewise isometries have zero topological entropy, Ergod. Th. \& Dynam. Sys. 21 (2001), 1371-1377.

[BTW] P. Bak, C. Tang, K. Wiesenfeld, Self-Organized Criticality: An explenation of 1/f-noise, Phys. Rev. Lett. 59, Num 4 (1987), 381-384.

P. Bak, C. Tang, K. Wiesenfeld, Self-Organized Criticality, Phys. Rev. A 38, Num 1 (1988), 364-374.

[BC] T. Bogenschütz, H. Crauel, The Abramov-Rokhlin Formula, Ergodic theory and related topics, III (Gustrow, 1990), 32-35, Lecture Notes in Math., 1514, Springer, Berlin, 1992.

[Bo] R. Bowen, Entropy for group endomorphisms and homogeneous spaces, Trans. A.M.S. 153 (1971), 401-414.

[Br] J. R. Brown, Ergodic theory and topological dynamics, Pure and Applied Mathematics, 70, Academic Press [Harcourt Brace Jovanovich Publ.], New YorkLondon (1976).

[BCK] Ph. Blanchard, B. Cessac, T. Krüger, What can we learn about SOC from Dynamical System Theory, J. Statist. Phys. 98 (2000), no. 1-2, 375-404.

B. Cessac, Ph. Blanchard, T. Krüger, Lyapunov exponents and transport in the Zhang model of self-organized criticality, Phys. Rev. E 64 (2001).

B. Cessac, Ph. Blanchard, T. Krüger J. L. Meunier, Self-Organized Criticality and Thermodynamic Formalism, J. Statist. Phys. 115 (2004), 1283-1326.

[DGS] M. Denker, C. Grillenberger, K. Sigmund, Ergodic theory on compact spaces, Lecture Notes in Math., 527. Springer-Verlag, Berlin-New York (1976).

[DR] D. Dhar, R. Ramasway, Exactly solved Model of Self-Organized Critical Phenomena, Phys. Rew. Let. Vol 63, Num 16 (1089), 1659-1662.

[GD] A. Giacommetti, A. Dias-Guilera, Dynamical properties of the Zhang model of self-organized criticality, Phys. Rev. Let. E, 58, no. 1 (1998).

[H] J. E. Hutchinson, Fractals and self-similarity, Indiana University Math. Journal, 30 no.5 (1981), 713-747.

[J] H. J. Jensen, Self-Organized Criticality: Emergent Complex Behavior in Physical and Biological systems, Cambridge Lecture Notes in Physics 10, Cambridge University Press (1998).

[K] A. Katok, Lyapunov exponents, entropy and periodic orbits for diffeomorphisms, Inst. Hautes Etudes Sci. Publ. Math., 51 (1980), 137-173.

$[\mathrm{KH}]$ A. Katok, B. Hasselblatt, Introduction to the Modern Theory of Dynamical Systems, Cambridge University Press (1995). 
Dynamics and entropy of SOC

[KS] A. Katok, J.-M. Strelcyn, Invariant manifolds, Entropy and Billiards; Smooth Maps with Singularities, Lecture Notes in Math., 1222, Springer, Berlin, 1986.

[KK] B. V. Kozelov, T. V. Kozelova, Cellular automata model of magnetosphericionospheric coupling, Annales Geophysicae, 21 (2003), 1931-1938.

[KR1] B. Kruglikov, M. Rypdal, A piece-wise affine contracting map with positive entropy, ArXiv: math.DS/0504187

[KR2] B. Kruglikov, M. Rypdal, Entropy via multiplicity, ArXiv: math.DS/0505019

[LY] F. Ledrappier, L.-S. Young, The metric entropy of diffeomorphisms, I-II, Ann. of Math. (2) 122 (1985), no. 3, 509-539; 540-574.

[M] P. A. P. Moran, Additive functions of intervals and Hausdorff measure, Proc. Camb. Phil. Soc., 42 (1946), 15-23.

[P1] Y. Pesin, Dynamical systems with generalized hyperbolic attractors: hyperbolic, ergodic and topological properties, Ergod. Th. \& Dynam. Sys. 12 (1992), 123151.

[P2] Y. Pesin, Dimension theory in dynamical systems, Chicago Lect. in Math. Ser., The University of Chicago Press (1997).

[Pt] K. Petersen, Chains, entropy, coding, Ergod. Th. \& Dynam. Sys. 6 (1986), no. $3,415-448$.

[PY] M. Pollicott, M. Yuri, Dynamical systems and ergodic theory, London Mathematical Society Student Texts 40, Cambridge University Press (1998).

[Ru] D. Ruelle, Chaotic evolution and strange attractors, Lezioni Lincee, Cambridge University Press (1989).

[R] M. Rypdal, Dynamics of the Zhang model of Self-Organized Criticality, Master Thesis in mathematics, University of Troms- 2004, e-printed: http://www.math.uit.no/seminar/preprints.html

[ST] J. Schmeling, S. Troubetzkoy, Dimension and invertibility of hyperbolic endomorphisms with singularities, Ergod. Th. \& Dynam. Sys. 18 (1998), 12571282.

[Z] H.Y. Zhang, Scaling theory of Self-Organized Criticality, Phys. Rev. Lett. 63 Num 5 (1988), 470-473. 